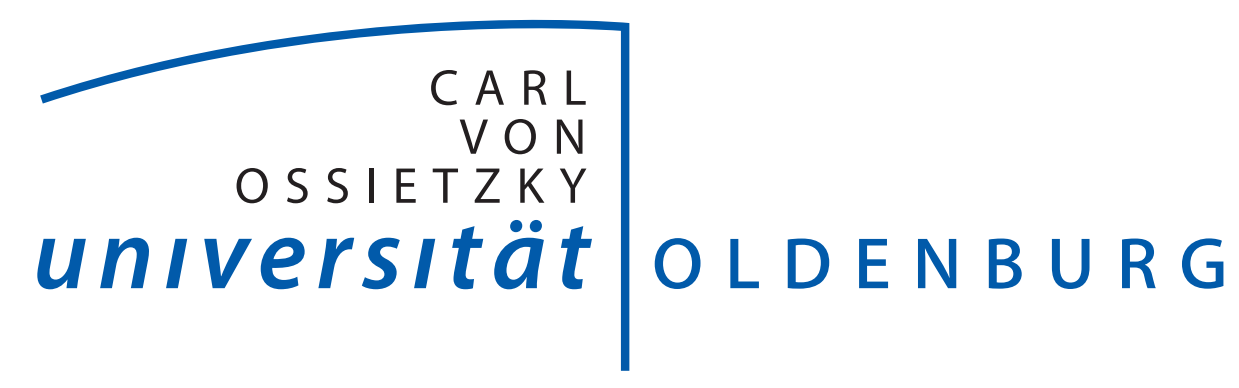

\title{
Optimized Support Vector Regression Algorithm-based Modeling of Ship Dynamics
}

\begin{abstract}
A dissertation accepted by the School of Computing Science, Business Administration, Economics and LaW of the Carl von Ossietzky University Oldenburg (Germany) to obtain the Degree AND title
\end{abstract}

Doctor of ENGINEERING (Dr.-ING.)

by Man Zhu, M.Sc.

BORN ON OCTOBER 8TH, 1989 IN WuHAN, ChINA

REVIEWERS:

Prof. Dr.-Ing. Axel Hahn

Prof. Dr. Yuangiao Wen

DATA OF DisPUTATION: $\mathbf{1 7 \cdot 1 2 . 2 0 1 8}$ 


\begin{abstract}
This thesis deals with the problem of how to derive a simplistic model feasible for describing the dynamics of ships for maneuvering simulations employed to study maritime traffic and to provide ship models for simulation-based engineering testbeds. The model should be expressed in a simple form with satisfactory accuracy as well as fast computation in simulations.

The problem of deriving a ship dynamic model is addressed first with the modification and simplification of a complex vectorial representation in 6 degrees of freedom (DOF). The 6 DOF dynamic model is simplified through several pieces of reasonable assumptions that are exampled as ships moving in the horizontal plane in the ideal fluid, the uniform distribution of ship masses, the port-starboard symmetry. In the process of simplification, the trade-off between the accuracy and the possibility of estimation of the simplified model is regarded as the key criteria. Consequently, a 3 DOF dynamic model in a simple form with four terms for capturing surge motions and eight terms for steering motions is found, in which the reduced-term version of the steering model expressed in five terms is further obtained under the consideration of the improvement of the model accuracy. The simplified ship dynamic model is derived to be applicable for different types of ships, which is different from the studied 3 DOF dynamic model that are used individually for one specified case/ship. That means the proposed solution on creating a 3 DOF ship dynamic model can be used for different types of ships only with minor modifications.
\end{abstract}

Solving the problem of deriving the ship dynamic model requires the estimation of parameters in the model through a suitable technique. With the limitation of the available equipment and the acquired observations applicable to estimated parameters, this thesis determines to adopt the system identification in combination with full-scale ship trail tests, e.g., standard zigzag maneuvers, to estimate parameters in the simplified ship dynamic model.

A literature review on identification methods in maritime engineering suggests the 
commonly-used least square method, the Kalman filter method and their modified versions with effective performance. But least square based identification methods aiming at minimizing a squared norm is sensitive to outliers corrupted by data, and the accuracy of results and convergence of Kalman filter method based identification methods are adversely influenced by the values of parameters predetermined depending on experts. Comparatively, these barriers would not happen to support vector machine (SVM) based identification methods due to it complies with the criteria of structural risk minimization, incorporates with the kernel function, and works on a finite set of data, etc. One deficiency of SVM based identification methods, i.e., the particular set of structural parameters such as the insensitivity factor, the regularization parameter, and kernel parameters, should be remedied to well control the trade-off between the empirical risk and the confidential interval. The artificial bee colony algorithm is applicable to complete this task, which shows superior optimization performance over the commonly-used cross-validation method (CV) and particle swarm optimization algorithm (PSO) with the advantage of fewer control parameters.

Finally, the approach on modeling ships' dynamics based on an artificial bee colony algorithm (ABC) optimizing the least square support vector regression algorithm (LS-SVR) based identification method is evaluated. Data extracted from a set of steady-state and zigzag maneuvers are applied to test the effectiveness of the simplified ship dynamic model and to validate the performance of the optimized LS-SVR in identifying the model. In the evaluations, the further simplified model with five terms presents higher accurate than the first order Nomoto models, which is also valid for capturing the steering dynamics of a real vessel. Through studies on estimation methods, LS-SVR based identification method shows more robust than the least square method to outliers. LS-SVR based identification method with the regularization parameter optimized by $\mathrm{ABC}$ performs superiorly. The time complexity of the ABC-LSSVR identification method is $O\left(N^{2}+l^{3}\right)$ with the number of samples applied for ABC optimization $(N)$ and the number of support vectors in LS-SVR (l), which is further demonstrated to be 
consistent with the evaluation results that the cost time increases with the increasing of the number of samples. Consequently, the whole evaluation results show a valid model in a simple form for describing the dynamics of different types' ships and also validate the performance of the proposed parameter estimation method. 
Man Zhu

\section{ZUSAMMENFASSUNG}

Diese Dissertation behandelt das Problem der Ableitung von vereinfachten Schiffsdynamikmodellen zur Verwendung in Manövrier Simulationen. Diese Schiffsmodelle sollen für simulationsbasierte Engineering-Testplattformen bereitgestellt werden um den Seeverkehr zu untersuchen. Die abgeleiteten Modelle sollen eine zufriedenstellende Genauigkeit aufweisen und eine schnelle Berechnung in Simulationen erlauben.

Das Problem, ein dynamisches Schiffsmodell abzuleiten, wird zunächst mit der Modifikation und Vereinfachung einer komplexen vektoriellen Darstellung in 6 Freiheitsgraden (DOF) behandelt. Das dynamische 6-DOF-Modell wird durch einige nachvollziehbare Annahmen, zum Beispiel das Schiffe in der Horizontalenebene sich in einem Idealfluid befinden, eine gleichmäßige Verteilung der Schiffsmassen, die Backbord-Steuerbord-Symmetrie. Der Kompromiss zwischen der Genauigkeit und der Möglichkeit der Schätzung des vereinfachten Modells wird als Schlüsselkriterium angesehen. Folglich wird ein dynamisches 3-DOF-Modell in einer einfachen Form gefunden, das verwendet wird, um Stoßbewegungen und Lenkbewegungen gleichzeitig zu erfassen. Dieses für Schiffstypen anwendbare Modell, das auf der Grundlage der Systemidentifikationstechnik erstellt wird, unterscheidet sich von den untersuchten dynamischen Modellen mit 3 DOF, welche nur für ein bestimmtes Schiff verwendet werden können. Das bedeutet, dass die in dieser Arbeit vorgeschlagene Lösung zum Erstellen eines dynamischen 3 DOF Schiffsmodells für verschiedene Schiffstypen mit wenigen Änderungen am Versuchsaufbau nützlich ist.

Um das Problem der Ableitung des Schiffsdynamikmodells zu lösen, müssen die Parameter im Modell durch eine geeignete Techniken geschätzt werden. Mit der Beschränkung der Ausrüstung und den erhaltenen Beobachtungen, die auf Schätzungen der Parameter anwendbar sind, bestimmt diese These, die Systemidentifikation in Kombination mit vollständigen Schiffstests zu verwenden, z. B. Standard-Zickzackmanöver, um Parameter in dem vereinfachten 
Schiffsdynamikmodell zu schätzen.

Eine Literaturrecherche zu Identifizierungsmethoden in der Schifffahrtstechnik schlägt die häufig verwendete Methode der kleinsten Quadrate, die Kalman-Filtermethode und ihre modifizierten Versionen mit effektiver Leistung vor. Aber kleinste quadratische Identifizierungsmethoden, die darauf abzielen, eine quadratische Norm zu minimieren, sind empfindlich gegenüber durch Daten verfälschte Ausreißer, und die Genauigkeit der Ergebnisse und die Konvergenz von Kalman-Filter basierten Identifizierungsmethoden werden durch die Parameterwerte in Abhängigkeit von Experten negativ beeinflusst. Im Vergleich dazu würden diese auf Support Vector Machine (SVM) basierten Identifizierungsmethoden unterstützen, da sie den Kriterien der strukturellen Risikominimierung entsprechen, mit der Kernfunktion inkorporiert sind und auf einem endlichen Satz von Daten arbeiten, und so weiter. Ein Mangel der SVM basierte Identifizierungsmethoden, d.h. die besondere Einstellung von Strukturparametern wie Insensitivitätsfaktoren, Regularisierungsparameter und Kernparameter, sollten behoben werden, um eine globale Optimierung zu gewährleisten. Der Artificial Bee Colony algorithm $(\mathrm{ABC})$ ist anwendbar, um diese Aufgabe zu erfüllen, die eine überlegene Optimierungsleistung gegenüber der üblicherweise verwendeten Cross-Validation method (CV) und Particle Swarm Optimization algorithm (PSO) mit der Nutzung von weniger Steuerparametern zeigt.

Schließlich wird der Ansatz zur Modellierung der Schiffsdynamik basierend auf Identifikationsverfahren von Least Square Support Vector Regression algorithm (LS-SVR), die mit dem $\mathrm{ABC}$ optimiert sind, evaluiert. Die aus einem Datensatz von stationären und zickzackförmigen Manövern extrahierten Daten werden angewendet, um die Wirksamkeit des vereinfachten Schiffsdynamikmodells zu testen und die Leistungsfähigkeit des optimierten LS-SVR bei der Identifizierung des Modells zu validieren. In den Auswertungen bietet das weiter vereinfachte Modell mit fünf Bedingungen eine höhere Genauigkeit als die Nomoto-Modelle erster Ordnung, was auch für die Erfassung der Lenkdynamik eines realen Schiffes gilt. Durch Forschungen über Schätzmethoden wurde gezeigt das LS-SVR basierte Identifikationsverfahren 
deutlich robuster gegenüber Sonderfällen sind als die Verfahren der kleinsten Quadrate. LS-SVR basierte Identifikationsverfahren mit dem durch den $\mathrm{ABC}$ optimierten Regularisierungsparameter erbringen eine bessere Leistung. Die Zeitkomplexität des ABC-LSSVR Identifikationsverfahrens ist $O\left(N^{2}+l^{3}\right)$ mit der für die ABC Optimierung $(N)$ angewendeten Stichprobenzahl und der Anzahl von Support Vectors in LS-SVR (l). Es wurde weiter gezeigt, diese sie konsistent mit den Bewertungsergebnissen ist und, dass die Kostenzeit mit einer höheren Anzahl von Proben zunimmt. Infolgedessen zeigen die Bewertungsergebnisse dass ein vereinfachtes gültiges Schiffsdynamikmodell für verschiedene Schiffstypen gefunden werden kann mit der vorgeschlagenen Parameter-Schätzungsmethode. 


\section{Acknowledgments}

There are many people who have earned my gratitude for their valuable contributions to my time at Oldenburg University.

More specifically, I would like to thank my supervisor, Prof. Dr. -Ing. Axel Hahn, for his endless support and constant encouragement during my Ph.D. study in the SAMS program. He has given me inspiring guidance on how to conduct scientific research in modeling ship dynamics and system identification. Without his constant support and helpful guidance, this thesis would not have been possible. Axel is the funniest advisor and one of the smartest people I know. He always inspires me with his passionate attitude and forward-looking scientific insights. I would give Axel most of the credit for becoming the kind of scientist I am today.

Besides, I am so grateful to Prof. Yuanqiao Wen, my second supervisor, who was also my master supervisor. He led me into the maritime-related scientific research field and taught me how to identify a research question, find a solution to it, and finally publish the results in high-level journals. Even more than $7 \mathrm{~km}$ distance, he was still glad to provide insightful discussions and suggestions to me whenever I was in confusion of my research.

I am also thankful to the members of the doctoral examination committee, Prof. Dr. Martin Fränzle and Dr. Lars Weber for their great support, impressive discussion and invaluable advice.

I would also like to express my sincere gratitude to my friends and colleagues for helping me overcome difficulties from work and life. I do like to own my great gratitude to our secretary, Manuela Wüstefeld. I would never forget her warm-heart help and encouragement to me during these years especially the tough period when I was pregnant.

My very special gratitude goes out to all down at Research Fund from the Ministry of Science and Culture of Lower Saxony for the Graduate School of Safe Automation of Maritime Systems (SAMS) for funding the work.

Last but by no means least, I am grateful to my beloved husband, Wuqiang Sun, who has provided me through emotional support in my life. I am also grateful to my other family members 
who have supported me along the way. This dissertation would not have been possible without their warm love, continued patience, and endless support. 


\section{Contents}

Abstract

ZUSAMMENFASSUNG $\quad$ iv

ACKNOWLEDGMENTS vii

$\begin{array}{ll}\text { CONTEnts } & \text { ix }\end{array}$

Listing of AbBREVIATIONS $\quad$ xiii

Listing OF Figures $\quad$ xv

Listing OF TABLES $\quad$ xvii

1 INTRODUCTION 1

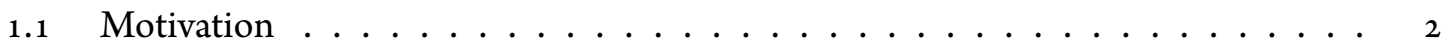

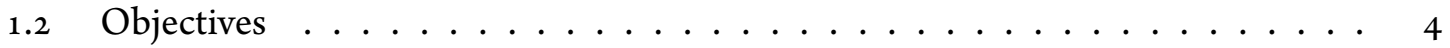

1.3 Main Contributions . . . . . . . . . . . . . . . . 5

1.4 Thesis Outline .......................... 6

2 Requirements of a Simplified Ship Dynamic Model $\quad 8$

2.1 Requirements on the Modeling of Ship Dynamics . . . . . . . . . . . . . 8

2.2 Requirements on the Identification Method . . . . . . . . . . . 10

3 Related Work 12

3.1 Ship Dynamic Model . . . . . . . . . . . . . . . . . . . . . . 13

3.1.1 General Remarks: Reference Frames and Notations . . . . . . . . 13 
3.1.2 Classification of Ship Dynamics . . . . . . . . . . . . 15

3.1.3 Introduction to Ship Dynamic Models . . . . . . . . . . . . 16

3.1.3.1 Rigid-body Equations of Motion . . . . . . . . . . . 19

3.1.3.2 Abkowitz Model . . . . . . . . . . . . . . . 20

3.1.3.3 MMG Model. . . . . . . . . . . . . . . . 21

3.1.3.4 Vectorial Representations . . . . . . . . . . . 23

3.1.4 Requirement Fulfillment Summary . . . . . . . . . . . . . . 28

3.2 System Identification . . . . . . . . . . . . . . . . . . . 29

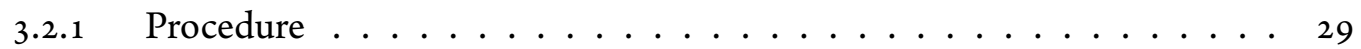

3.2.1.1 The Observed Data . . . . . . . . . . . . . . 29

3.2.1.2 The Set of Models or the Model Structure . . . . . . . . . 30

3.2.1.3 Identification Method . . . . . . . . . . . . 31

3.2.1.4 Model Validation . . . . . . . . . . . . . . . 31

3.2.2 Identification of the Ship Dynamic Model . . . . . . . . . . . 31

3.2.3 Support Vector Machines . . . . . . . . . . . . . . . 34

3.2.3.1 Linear Support Vector Classification Algorithm . . . . . . . 35

3.2.3.2 Nonlinear Support Vector Classification Algorithm . . . . . . 39

3.2.3.3 Support Vector Regression Algorithm . . . . . . . . . . . 40

3.2.3.4 Least Square Support Vector Regression Algorithm . . . . . . 41

3.2.4 Requirement Fulfillment Summary . . . . . . . . . . . . . . . . 42

3.3 Objective Coverage Summary . . . . . . . . . . . . . . . . . . 44

4 Modeling of Ship Dynamics $\quad 46$

$4.1 \quad 3$ DOF Ship Dynamic Model . . . . . . . . . . . . . . . . 47

4.2 Actuator Forces and Moments . . . . . . . . . . . . . . . . 49

4.3 Simplified Ship Dynamic Model . . . . . . . . . . . . . . . . . . . 51

$4.3 .1 \quad$ Surge Model . . . . . . . . . . . . . . . . . . 51

$4.3 .2 \quad$ Steering Model $\ldots \ldots \ldots \ldots \ldots \ldots$

$4.3 .3 \quad$ Nomoto Models . . . . . . . . . . . . . . . . . . . 52

5 ABC-LSSVR based Parameter Estimation Method 55

$5.1 \quad$ LS-SVR based Parameter Estimation Method . . . . . . . . . . . . . . 56

5.1 .1 Introduction to the Method . . . . . . . . . . . 57

5.1.2 Construction of Samples for Identification . . . . . . . . . . 58

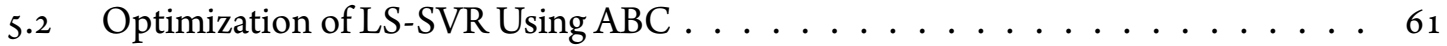


5.2.1 Key Stages in Artificial Bee Colony Algorithm . . . . . . . . . . . 61

5.2 .1 .1 Initialization . . . . . . . . . . . . 61

5.2.1.2 The Employed Bees Stage . . . . . . . . . . . . 62

5.2.1.3 The Onlooker Bees Stage . . . . . . . . . . . 63

5.2.1.4 The Scout Bees Stage . . . . . . . . . . . . . . . . 63

5.2.2 Procedure of ABC-LSSVR for Identification . . . . . . . . . 63

5.2 .3 Complexity Analysis . . . . . . . . . . . . . . . . . . 65

6 Implementation of the SIMSD Prototype $\quad 67$

6.1 SIMSD System Design . . . . . . . . . . . . . . . . . 67

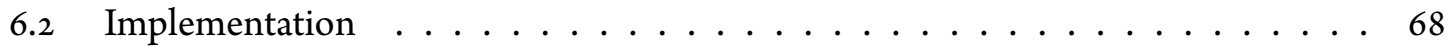

7 Evaluation $\quad \mathbf{7 1}$

7.1 Design of Case Studies . . . . . . . . . . . . . . . . 72

7.2 Case 1: Selection and Comparison between LS and LS-SVR . . . . . . . 72

7.2 .1 Data Processing . . . . . . . . . . . . . . 73

7.2.2 Results and Analysis . . . . . . . . . . . . . . . 73

7.3 Case 2: Improvement of RLS Using LS-SVR . . . . . . . . . . . . 76

$7 \cdot 3.1 \quad$ Data Processing . . . . . . . . . . . . . 76

$7 \cdot 3.2$ Selection of Samples for LS-SVR $\ldots \ldots \ldots \ldots \ldots$

$7 \cdot 3.3 \quad$ Identification Results . . . . . . . . . . . . . . . 79

7.3 .4 Validation ....................... 82

7.4 Case 3: Parameter Estimation of the Simplified Ship Dynamic Model Using ABC-

LSSVR ........................ 82

$7 \cdot 4.1 \quad$ Data Processing ...................... 83

7.4.2 Identified Models Using ABC-LSSVR . . . . . . . . . . . . 84

$7 \cdot 4.3$ Validation ....................... 86

7.4.4 Comparison of Three Optimized LS-SVRs . . . . . . . . . . . . 88

7.4.5 Comparison of Steering Models . . . . . . . . . . . . . . . . 91

7.4.6 Special Considerations: Further Simplification of the Nonlinear Steering Model . . . . . . . . . . . . . . . . . 93

7.4.7 Time Complexity Analysis . . . . . . . . . . . . . 95

7.5 Case 4: Experimental Validation of the Further Simplified Dynamic Model ․ . . 98

7.5.1 Data Acquisition and Processing . . . . . . . . . . . . 98

7.5 .2 Identification Results and Validation . . . . . . . . . . . 100 
7.6 Summary of Objective Coverage and Requirement Fulfillment . . . . . . . 101

8 Conclusions $\quad 110$

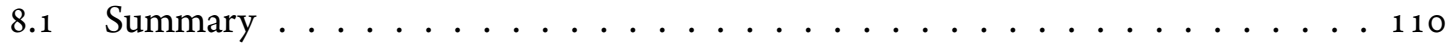

8.2 Outlook ........................... 112

A Appendix $\quad 114$

A.1 Least Square Method . . . . . . . . . . . . . . . . . . . . 114

A.2 Recursive Least Square Method . . . . . . . . . . . . . . . 115

A.3 A Linear Steering Model . . . . . . . . . . . . . . . . . 115

A.4 A 4 DOF Nonlinear Model of a Large Container Ship . . . . . . . . . . . . 117

$\begin{array}{lr}\text { REFERENCES } & 119\end{array}$ 


\section{Listing of Abbreviations}

DOF Degree of Freedom

AIS Automation Identification System

ECDIS Electric Chart Display and Information System

GMDSS Global Maritime Distress and Safety System

SVR Support Vector Regression Algorithm

ABC Artificial Bee Colony Algorithm

PSO Particle Swarm Optimization Algorithm

CV Cross-Validation Method

IMO International Maritime Organization

GBS Goal-based Standards

EEDI Energy Efficiency Design Index

MUNIN Maritime Unmanned Navigation through Intelligence in Networks

PMM Planar Motion Mechanism

CFD Computational Fluid Dynamics

LS Least Square Method

RLS Recursive Least Square Method

$\operatorname{SVM}(\mathrm{s}) \quad$ Support Vector Machine(s)

LSSVR Least Square Support Vector Regression Algorithm

MMG Maneuvering Modeling Group

SNAME Society of Naval Architects and Marine Engineers

EKF Extended Kalman Filtering Algorithm

GA Genetic Algorithm

GS Global Search Algorithm

SA Simulated Annealing Algorithm

ASV Autonomous Surface Vehicle 


$\begin{array}{ll}\text { NN } & \text { Neural Network Method } \\ \text { ML } & \text { Maximum Likelihood Method } \\ \text { SRM } & \text { Structural Risk Minimization } \\ \text { SVC } & \text { Support Vector Classification Algorithm } \\ \text { SMO } & \text { Sequential Minimal Optimization } \\ \text { MATLAB } & \text { MATrix LABoratory } \\ \text { VSZZ } & \text { Very Small zigzag Maneuvers } \\ \text { MSE } & \text { Mean Squared Errors between Prediction and Simulation Results }\end{array}$




\section{Listing of Figures}

3.1.1 Illustration of reference frames. . . . . . . . . . . . . . . . 14

3.1.2 Description of planar motion variables. $x_{\delta}$ is the longitudinal moment arm from the center of rotation to the pivot point of the thruster/propulsion. . . . . . 15

3.1.3 Illustration of the classification of ship dynamics (Perez and Fossen 2006). . . . 16

3.2.1 The system identification procedure . . . . . . . . . . . . . 30

3.2.2 Basic framework of the identification of ship dynamic model. . . . . . . . . 32

3.2 .3 Graphical illustration of SVR. . . . . . . . . . . . . . 41

5.2.1 Graphical representation of the ABC-LSSVR. . . . . . . . . . . . . . . 64

6.1.1 Component diagram of the SIMSD system. . . . . . . . . . . . . . 68

6.2.1 Snapshots of functions and files used for SIMSD implementation. . . . . . . . 70

7.2.1 Time series of the rudder and heading angles. The blue lines are the actual rudder angle in the upper sub-figure of the $20^{\circ} / 20^{\circ}$ zigzag maneuver, and the heading angle in the bottom sub-figure of the $20^{\circ} / 20^{\circ}$ zigzag maneuver. The red lines are the actual rudder angle in the upper sub-figure of the $30^{\circ} / 30^{\circ}$ zigzag maneuver, and the heading angle in the bottom sub-figure of the $30^{\circ} / 30^{\circ}$ zigzag maneuver. . 74

7.2.2 Prediction results of the first order linear models identified by LS and LS-SVR, respectively. (a) measured yaw rate and predicted yaw rate, (b) measured heading angle and predicted heading angle, (c) residual of the yaw rate by calculating the difference between measurements and predictions, (d) residual of the heading angle obtained by calculating the difference between measurements and predictions. 75

7.2.3 Statistical validation of the identified first order linear models. The left sub-figure is the normalized auto-correlation, the middle one is the normalized cross-correlation, the right one presents the residual analysis. . . . . . . . . . . 76 
7.2.4 Prediction results of the first order nonlinear models identified by LS and LSSVR, respectively. (a) measured yaw rate and predicted yaw rate, (b) measured heading angle and predicted heading angle, (c) residual of the yaw rate by calculating the difference between measurements and predictions, (d) residual of the heading angle obtained by calculating the difference between measurements and predictions. . . . . . . . . . . . . . . . 77

7.2.5 Statistical validation of the identified first order nonlinear models. The left subfigure is the normalized auto-correlation, the middle one is the normalized crosscorrelation, the right one presents the residual analysis. . . . . . . . . . 78

7.3.1 Simulation of the $20^{\circ} / 20^{\circ}$ zigzag maneuver. The top sub-figure is the commanded rudder and the heading angles, the second one is the sway velocity, the third one is the yaw rate, the bottom is the resultant speed. . . . . . . . . . . . . . . 79

7.3.2 Convergence of LS-SVR in the condition of varying number and interval of samples. 80

7.3.3 Identification results of RLS and LSSVR-RLS. . . . . . . . . . . . . . . 81

7.3.4 Predictions of the $10^{\circ} / 10^{\circ}$ zigzag maneuver using the model identified by LSSVRRLS. The top sub-figure is the commanded rudder angle and the heading angle, the second sub-figure is the sway velocity, the third one is the yaw rate, the bottom sub-figure is the resultant speed. . . . . . . . . . . . . . . 83

7.4.1 Simulated data for the optimization and the identification of simplified dynamic models. The top sub-figure presents the surge speed and commanded/actual propeller shaft speed of the first straight line maneuver as described in Table 7.4.1, the middle sub-figure donates the heading and commanded/actual rudder angles of the $10^{\circ} / 10^{\circ}$ zigzag maneuver, the bottom sub-figure is the sway speed and yaw rate of the $10^{\circ} / 10^{\circ}$ zigzag maneuver. . . . . . . . . . . . . . . 85

7.4.2 Regression of the ABC-LSSVR. The upper sub-figure is the surge speed of the first straight line maneuver, the middle one is the sway speed of the $10^{\circ} / 10^{\circ} \mathrm{zigzag}$ maneuver, the lower one is the yaw rate of the $10^{\circ} / 10^{\circ}$ zigzag maneuver. $\quad \ldots . .87$

7.4.3 Predictions of ship dynamics using models identified by the ABC-LSSVR. The upper sub-figure is the surge velocity of the second straight line maneuver, the middle one is the sway speed of the $20^{\circ} / 20^{\circ}$ zigzag maneuver, the bottom one is the yaw rate of the $20^{\circ} / 20^{\circ}$ zigzag maneuver. . . . . . . . . . . . . . 88 
7.4.4 Comparison of the three ship dynamic models identified by CV-LSSVR, PSOLSSVR, and ABC-LSSVR, respectively. The top sub-figure is the predictions of surge velocity, the middle one is the predictions of sway velocity of the $20^{\circ} / 20^{\circ}$ zigzag maneuver, the bottom sub-figure is the predicted yaw rate of the $20^{\circ} / 20^{\circ}$ zigzag maneuver. . . . . . . . . . . . . . . . . 91

7.4.5 Predictions of $20^{\circ} / 20^{\circ}$ zigzag maneuver using different identified steering models. 93

7.4.6 Predictions of $20^{\circ} / 20^{\circ}$ zigzag maneuver using the further simplified steering model. The top sub-figure is the predictions of sway velocity, the bottom sub-figure is the predicted yaw rate . . . . . . . . . . . . . . . 96

7.4.7 The time consumed by the three estimation methods on identifying the surge model. 97

7.4.8 The time consumed by the three estimation methods on identifying the steering model. The top sub-figure presents the cost time of the sway model, the bottom sub-figure means the cost time of the yaw model. . . . . . . . . . . . 98

7.4.9 The MSE of the three identified surge models. . . . . . . . . . . . . . . 99

7.4.10The MSE of the three identified further simplified steering model.(a) the MSE of the identified sway models. (b) the MSE of the identified yaw models. . . . . . . 100

7.4.1 1 The estimated parameters of the three identified surge models. The top sub-figure is the parameter $a_{1}$, the middle sub-figure donates the parameter $a_{2}$, the bottom sub-figure means the parameter $a_{3} \ldots \ldots \ldots \ldots 10 \ldots \ldots$

7.4.12 The estimated parameters of the three identified further simplified sway models.

(a) parameter $b_{v}$, (b) parameter $b_{r}$, (c) parameter $b_{|v| r}$ (d) parameter $b_{\delta} . \ldots{ }_{102}$ 7.4.13 The estimated parameters of the three identified further simplified yaw models.

(a) parameter $c_{r}$, (b) parameter $c_{v}$, (c) parameter $c_{|v| r}$, (d) parameter $c_{\delta} . \ldots . . .103$

7.5 .1 The I-Nav- II vessel. . . . . . . . . . . . . . . . . . . . . . . 104

7.5.2 Experimental data of the $10^{\circ} / 10^{\circ}$ zigzag maneuver on the I-Nav-II vessel. . . . . . 105

7.5 .3 Predictions of the $20^{\circ} / 20^{\circ}$ zigzag maneuver. . . . . . . . . . . 106

7.5 .4 Errors of the $20^{\circ} / 20^{\circ}$ zigzag maneuver. . . . . . . . . . . . . 107

7.5.5 Predictions of the $25^{\circ} / 25^{\circ}$ zigzag maneuver. . . . . . . . . . . . 108

7.5 .6 Errors of the $25^{\circ} / 25^{\circ}$ zigzag maneuver. . . . . . . . . . . . . . 109 


\section{Listing of Tables}

3.1.1 Notations from SNAME. . . . . . . . . . . . . . . . . . . . 14

7.1 .1 Description of the case studies. . . . . . . . . . . . . 72

7.2.1 Identified maneuvering indices of the first order Nomoto models. . . . . . . . 73

7.3.1 Parameters of the Merchant Ship Mariner class. . . . . . . . . . . . . . . 77

7.3.2 Identification results of RLS and LSSVR-RLS. . . . . . . . . . . . 82

7.4 .1 The scheme of ship maneuvers. . . . . . . . . . . . . 84

7.4.2 The best regularization parameter and MSE . . . . . . . . . . . 86

7.4.3 Parameters estimated by ABC-LSSVR . . . . . . . . . . . . . 86

7.4.4 Comparison of the computational time, the best regularization parameter, and MSE. 89

7.4.5 Parameters of the surge model estimated by CV-LSSVR, PSO-LSSVR, and ABC-

LSSVR, respectively. . . . . . . . . . . . . . . . 90

7.4.6 Parameters of the sway model estimated by CV-LSSVR, PSO-LSSVR, and ABC-

LSSVR, respectively. . . . . . . . . . . . . . . . 90

7.4.7 Parameters of the yaw model estimated by CV-LSSVR, PSO-LSSVR, and ABC-

LSSVR, respectively. . . . . . . . . . . . . . . . . . . 90

7.4.8 Identification results of Nomoto models. . . . . . . . . . . . . . . . . 92

7.4 .9 Estimated parameters of the sway model. . . . . . . . . . . . . . . 95

7.4 .10 Estimated parameters of the yaw model. . . . . . . . . . . . . . 95

A.4.1 Principal particulars of the container ship. . . . . . . . . 118 


\section{1 \\ Introduction}

In the past few decades, the shipping industry has gone through a series of technological advancements and has brought in massive digitization of machinery and equipment as well. The digital equipment used in modern ships, for instance, are Automation Identification System (AIS), Electronic Chart Display and Information System (ECDIS), Integrated Bridge Systems, Automatic Radar Plotting Aids, Long Range Identification and Tracking, Global Maritime Distress and Safety System (GMDSS) and several other sophisticated electronic navigational tools. Despite highly advanced equipment utilized in modern ships, maritime accidents related to navigation primarily due to human error during ship navigation continue to happen. The prime reason is that the advanced equipment systems battalized individually add a burden of work to mariners (i.e., too much information available (Denker 2014)) even though they may make navigation convenient and precise. Under the realization of this condition, the International Maritime Organization (IMO) decided to include a well-defined strategy to integrate new and existing navigational tools together effectively for enhancing the handling and safety of ships at sea along with the protection of the marine environment. Consequently, the e-Navigation strategy is proposed and defined as "the harmonized collection, integration, exchange, presentation and analysis of marine information on board and ashore by electronic means to enhance berth to berth 
navigation and related services for safety and security at sea and protection of the marine environment." (IMO 2014).

The major aim of e-Navigation is to enhance navigation safety of ships while simultaneously reducing the burden on navigational mariners. A well-coordinated and systematic system under e-Navigation would considerably increase the efficiency of ships not only at sea but also at the ports. Moreover, the global standardization of such a system would reduce complexity in ships' operation and substantially improve the safety of ships at sea. Since then, several maritime organizations both public and private along with IMO dedicate towards developing a robust e-Navigation system within the maritime international framework.

\subsection{Motivation}

One issue concerning robust e-Navigation technologies is their functional safety and reliability. As maritime transportation can be understood as a sociotechnical system, this issue ought to be studied through simulation-based testbeds (Hahn, Gollücke, Buschmann, and Schweigert 2015). As reported in (Hahn 2014), a modeling and simulation toolset named HAGGIS (Hybrid Architecture for Granularly, Generic and Interoperable Simulations) is a co-simulation system for the evaluation of e-Navigation concepts and systems by providing a maritime traffic simulator, a n-body simulator, and services to detect rare events of failure.

Within components of HAGGIS, each one has its functionality and works interactively. Instance for well understanding is the maritime traffic simulator (MTS) which is a flexible and usable maritime traffic simulation for implementing, executing and observing the behavior of multiple vessels in a realistic context (Hahn 2014). The simulated traffic information can be not only used for statical analysis but also transformed to other simulators who require it. For every vessel involved in MTS, its behavior comprising traffic information is animated by simultaneously using the dynamic model, the kinematic model, and the setting of the navigation environment. The traffic information simulated by MTS is the paramount supplement in which the dynamic model and kinematic model play a crucial role. In particular, more attention should be paid to the ship dynamic model, because the kinematic model is functional in combination with the outputs of the dynamic model. Besides, another noticeable point is the requirement of dynamic models for ships that implies every ship needs a compatible dynamic model applicable for capturing its unique motion characteristics. For this point, it would be beneficial if one model can be applicable for describing ships' motions with minor modification such as changing parameters but retaining the model structure. 
Generally, the ship is reasonably assumed as a rigid-body object so that its dynamic model is derived based on Newton's second law of motion (Fossen 2011). Typical and widely-used examples are the Abkowitz model built in Taylor series expansion (Lewis 1988), Maneuvering Modeling Group (MMG) model also called modulus model (Kobayashi, Kagemoto, and Furukawa 1995), Fossen's vectorial representation model additionally regarded as modulus model in vector-matrix form (Fossen 2011 ), and response model also called Nomoto model (Åström 1980). The former three models with the ability of completely capturing ship's dynamics are complicated and nonlinearly coupling in the use of a number of parameters. Major modification consuming much time and finance is required to adopt and switch one of these models among different types of ships due to unignorable differences existing in the hull, propeller, and rudder among various ships. Recall the above mentioned noticeable point about the requirement of dynamic models for ships, the former three models are not desirable fit. Trough the studies on the Nomoto model, it is reported to be applicable for control design especially the autopilot design in maritime engineering due to its simple structure and easy comprehension (Luo and Cong 2016) but its ability of capturing 1 DOF motions in yaw direction is not so expected to describe ship dynamics in particular used in ship's motion simulation such as the MTS. Therefore, the task of how to match the requirement for the establishment of one dynamic model for different types of ships is deserved considerable study. This task envelops two sub-tasks, i.e., the determination of model structure, and the estimation of parameters in the determined model.

Taking Newton's second law of motion and relatively derived models into account, the structure of the ship dynamic model is basically deterministic but requiring additional modification to get an appropriate model for ships. For another task in estimating parameters involved in the determined model, an adequate solution is required. Even though four core methods reported in studies are extensively used for determining ship dynamic models, including captive model test with planar motion mechanism (PMM) (Rhee, Yoon, Sung, Kim, and Kang 2000), estimation with empirical formulas (Hirano 1981), numerical calculation based on computational fluid dynamics (CFD) (Racine and Paterson 2005), and system identification in combination with free-running model test or full-scale trial (Luo and Zou 2009), they are still underdeveloped due to their respective weakness such as scaling effects, demand of extreme computation, etc. explained as follows. The captive model test with PMM is applicable to obtain most parameters, but it has a remaining problem of scaling effects aroused by the difference of Reynolds' number between the real ship and the scaled model ship which makes the measured value of parameters not wholly reliable (Yoon and Rhee 2003). The estimation with empirical formulas is practical and straightforward. The formulas are built based on statistical analysis of a 
set of ships, so the estimation results are not precise for the latest type of ships which are not included in the database. The rest two estimation methods are both powerful methods, but the numerical calculation based on CFD always requires extreme computing power, and its validation dramatically depends on the quality and amount of the experimental data. Comparatively, system identification in combination with a free-running model test or full-scale trial avoids the scaling effect and is easy to be undertaken, but parameter drift unpredictably existing among parameters if there were too many parameters in a model would compromise the accuracy of identification results. Other than scaling effects, the demand for extreme computational power and uninvolved empirical calculation, the influence of parameter drift on estimation accuracy of ship dynamic model is tricky to be overcome. Hence, the derived ship dynamic model should be not only in an appropriate form but also in a parameter-reduced version for mitigating the parameter drift influence and improving the accuracy of simulation of ship motions.

The challenges required to be overcome can be summarized as follows. The ship dynamic model is paramount for the MTS but not available to capture dynamics of different types' ships with only minor modification. The estimation methods are unexpectedly associated with weaknesses negatively influencing the accuracy and reliability of the estimated model. Therefore, this thesis devotes to developing an appropriately simplified ship dynamic model with a small number of terms for different types of ships to overcome the described challenges.

\subsection{OBjectives}

After the description of the scientific antecedents and motivation, the goal of this thesis is to overcome the addressed challenges at the same time to answer the research question:

How to derive a simplistic model for the description of dynamics of ships for maritime traffic simulation?

The question is divided into two sub-questions answered by achieving corresponding objectives.

\section{Sub-question 1 : How to simplify the complex dynamic model for ships?}

Objective 1: Selection of an existing 6 DOF model as the fundamental model for model simplification and modification. Many forms of existing ship dynamic model serve the choice for model simplification and modification. But each one presents its merits and demerits while being applied. If the simplified version of the $6 \mathrm{DOF}$ model is expressed in a simple form with low order and satisfactory accuracy, where each term has clear physical interpretation, this kind 6 DOF model can be the fundamental model for model simplification and modification. 
Objective 2: Simplification of the model dependent on trade-offs between accuracy and complexity. From a theoretical point of view, the complexity of the selected 6 DOF model is reduced by introducing a set of reasonable assumptions. Besides, numerical simulation and analysis on the first simplified model facilitate further simplification of the model from a practical viewpoint.

Sub-question 2: How to estimate the parameters in the simplified ship dynamic model?

Objective 3: Comparison and selection of parameter identification methods. A variety of identification methods exist, including conventional and intelligent methods. In order to determine a suitable method for special cases such as the identification of ship dynamic model, the comparison of the methods and related analysis are carried out from a methodological point of view. The selected identification method should be robust and intelligent enough to provide reliable results.

Objective 4: Improvement of the parameter identification method. The identification method generally has twofold, i.e., advantages and disadvantages. The deficiency of the method is remedied by an effective solution which has not been used in the maritime domain so that the thesis contributes to improving the performance of the identification method.

Objective 5: Parameter estimation tests by using simulated and experimental data. The simplified ship dynamic model and improved identification method are tested from both theoretical and practical points of view. The way for obtaining the simplified dynamic model for ships is verified by using data extracted from simulated maneuvers and experimental maneuvers generated by different ships.

\subsection{Main Contributions}

This thesis is dedicated to developing a solution for overcoming the challenges described in the motivation part as well as achieving the objectives addressed above with the concentration on ship dynamic model structure and parameter estimation. The main contribution of this thesis is summarized as: "Development of an approach for modeling dynamics of different types' ships by firstly using the optimized identification method". The detailed statement of the contribution is presented as follows:

- Achieve the modeling of ship dynamics through simplifying a complicated and nonlinearly coupling dynamic model into a reduced-term model with relatively low complexity and acceptable accuracy based on pieces of reasonable assumptions and practical case studies, which is feasible for capturing different types of ship's dynamics. 
- Select a suitable estimation method according to the qualitative analysis of results with the application of experimental data.

- Improve performance and accuracy of the estimation method by using an intelligent algorithm, which is the first time to be adopted in the maritime domain.

\subsection{Thesis OutLine}

This thesis is comprised of eight chapters where the answer to the research question is presented through evaluation results, and the contribution is highlighted as well. After clarifying the challenges in Chapter 1 where the research question is proposed which could be answered by achieving the addressed objectives, the main contribution of this thesis is highlighted.

The requirements to the solution for deriving a simplistic ship dynamic model are identified in Chapter 2 where the requirements on the ship dynamic model and parameter estimation are presented in detail, respectively.

Chapter 3 introduces the state of the art of the ship dynamic model and system identification. Ship dynamic models including the Abkowitz model, MMG model, Fossen's vectorial representation model are concretely presented. In system identification, some related concepts are explained first. Then the application of system identification technique to identify ship dynamic model is stated with the following details about support vector machines (SVMs). In the end, two relevant parts are summarized as the foundation for the solution proposed in this thesis.

Chapter 4 discusses the modeling of ship dynamics under the consideration of reduction of the complexity of existing 6 DOF models and the identifiability of the simplified model. The typical 6 DOF ship dynamic model expressed in vectorial representation is accounted as the fundamental model for simplification. The forces and moments acting on the ship are calculated by using the function of rudder deflection and propeller shaft speed. Consequently, a 3 DOF ship dynamic model comprised by the decoupling surge model and steering model is obtained.

Chapter 5 pays attention to the proposed identification method, i.e., ABC-LSSVR (least square support vector regression algorithm). The LS-SVR based identification method is first introduced. Then samples including training and validation samples are constructed for identification. To remedy the deficiency of LS-SVR, LS-SVR incorporated with ABC is discussed and the proposed ABC-LSSVR regarding the identification of the simplified ship dynamic model is demonstrated.

Following the implementation of the proposed solution in Chapter 6, the results of the numerical simulation and experimental study are presented and analyzed in Chapter 7. Case 
studies in terms of the particular purposes are carried out to get the results. The suitable identification method for identifying the simplified ship dynamic model, i.e., LS-SVR, is indicated through the comparison between LS and LS-SVR. Afterward, the advantage of LS-SVR, e.g., works with finite samples without knowing the initial values of parameters, is beneficial to some parameter identification methods requiring initial values of parameters, e.g., recursive least square method ( $R L S$ ), to improve identification results. The application of the optimized LS-SVR to estimate parameters in the simplified ship dynamic model is carried out. The identification results are analyzed in detail and motivate special considerations on further simplification of the nonlinear steering model. Furthermore, the experimental study on the I-Nav-II vessel verifies the special considerations.

This thesis is concluded in Chapter 8 where the future works needed to be further studied based on current works are pointed out. 


\section{2 Requirements of a Simplified Ship Dynamic Model}

Considering the previous introduction on the motivation and the required achievement of the objectives, this chapter defines the requirements related to the approach on developing a simplistic dynamic model of ships, which should be fulfilled at the end. The requirements of a simplified ship dynamic model are described in detail from two aspects concerning requirements on the modeling of ship dynamics and the identification method.

\subsection{Requirements on the Modeling of Ship Dynamics}

Requirements engineered in this part correspond to the first sub research question, which asks for a ship dynamic model established in a relatively simple form. The following requirements (expressed by $R_{1}, \ldots, R_{4}$ ) are explained to fulfill the objectives derived from the first sub research question.

- $\mathrm{R}_{1}$ : Each term in the model has a manifest physical interpretation. The determination of the external forces and moments acting on the operating ship is a much tough task after the 
expression of ship dynamic model is primarily defined based on Newton's second law of motion. Taylor-series expansion, modulus functions, and vectorial representation are three general ways to completely express forces and moments induced by ship hull, propeller, rudder, and interference. But to make the model applicable for describing the dynamics of different types of ships, differences of the hull/propeller/rudder are ought to be presented in the model. This requires the terms involved in the model with manifest physical interpretation (Browning 1991). The fulfillment of this requirement fits objectives 1-2.

- $\mathrm{R} 2$ : The model is expressed in a simple form with low order and a few terms. The effects of ship hull, propeller, and rudder on ship motion expressed in vector-matrix form is easy-understanding. The instance is the vectorial representation model containing low order but many terms, which shows superior advantages over the classical models such as the Abkowitz model and the MMG model while applied to model simulation (Fossen 2013). Dozens of coefficients are ought to be estimated by one time when applying the vectorial representation model, which is not a trivial job. The main reason is that the high correlation among a number of coefficients makes the parameter drift on estimated coefficients highly possible to occur (Luo 2016). If the model is switched to describe the dynamics of another different type of a ship, experiments on demand of much time and financial cost have to be done again. To ensure reliable identification of the ship dynamic model, the vectorial representation model expressed in a simple form with a few terms shall be considered, which is related to the achievement of objective 2 .

- $\mathrm{R}_{3}$ : The model is identifiable. Once the ship dynamic model is constructed, the parameters involved shall be estimated correctly. This implies the requirement that the model should be identifiable (Godfrey and DiStefano III 1985). On one hand, the parameters can be identified, which means the number of inputs and parameters is equivalent. On the other hand, no parameter drift effects exist. The effect of parameter drift existing in the identification results of the model makes the estimated parameters mathematical correct but physical incorrect. This phenomenon happens severely in the identification of a complicated ship dynamic model containing a number of parameters due to multicollinearity. To some degree, this requirement overlaps the second requirement and contributes to objectives 1-2.

- $\mathrm{R}_{4}$ : The model guarantees acceptable accuracy in describing ship dynamics. The identified ship dynamic model shall accurately predict ship dynamics which would be used to study properties of the ship for further intentions such as motion simulation for the MTS. This 
requires the finally obtained model guarantees accurate description of ship dynamics. One indicator, i.e., MSE, can illustrate how close the real data and the predicted data are (Hyndman and Koehler 2006). The smaller the value of the indicator is, the higher the accuracy of the model is. By analyzing the value of the indicator, whether the accuracy of the obtained model is acceptable or not can be assessed. This requirement contributes to objective 2.

\subsection{Requirements ON THE Identification Method}

After defining the structure of the ship dynamic model, parameters involved in the model are required to be estimated by using the system identification technique which corresponds to the second sub research question. The following present requirements (expressed by $\mathrm{R}_{5}, \ldots, \mathrm{R} 8$ ) on the identification method for providing reliably estimated parameters.

- $\mathrm{R}_{5}$ : The identification method is robust enough. In the application of system identification to identify ship dynamic model, one significant factor affecting the results is the data acquired from experiments of the ship (Moreno-Salinas, Chaos, de la Cruz, and Aranda 2013). Due to the existing of environmental factors (e.g., wind, currents, and waves.) and the use of sensors (e.g., compass, inertial measurement unit, and rudder indicator.), the data are inevitably corrupted by either the environmental disturbances or measurement noises. The signal processing technique, f.i., filtering, has been applied to pre-process the polluted data, but the disturbances and noises cannot be completely eliminated. Besides the pre-processing of the polluted data, the requirement on the robustness of the identification method shall be emphasized, which implies that the identification method shall be robust enough to defend the negative influence caused by the unfiltered environmental disturbances and measurements noises in the data. This requirement contributes to objectives 3-4.

\section{- R6: The identification method has no sensitivity to the predefined values of the parameters}

which are required to be estimated. The method is not sensitive to the initial estimation of the parameters of the ship dynamic model, referring to the potential research points proposed by ( $\mathrm{Fu}$ and $\mathrm{Li} 2013$ ). The main reason is that the initial estimation of parameters is significantly dependent on the prior knowledge of the characteristics of ship dynamics, which implies that it is extremely easy to obtain undesirable identification results even incorrect ones if the users have few pieces of knowledge of the system. This requirement 
contributes to objective 3 and objective 5 in flexibly applying for different cases/ships to identify the dynamic model.

- $\mathrm{R}_{7}$ : The identification method guarantees the global optimal solution. The physical correct results shall be consistent with the reality of ship dynamic characteristics. Meanwhile, attention shall be on the mathematical correct identification results in the process of parameter estimation of ship dynamic model. This requires the identification method with global optimization ability ( Fu and Li 2013). Parameters involved in the identification method shall be assigned with particular settings. This requirement contributes to objectives $4-5$.

- R8: The identification method should be of low complexity. In this thesis, the finally obtained model will be applicable to describe the dynamics of multiple vessels in simulation-based engineering testbeds, e.g., maritime traffic simulation. The overall speed of the simulation should be fast enough from a practical engineering application point of view. Relatively, the simulation speed is not impacted seriously by the complexity of the dynamic model but by the complexity of the identification method. So to ensure fast simulation, the identification method should be of low complexity. This requirement contributes to objective 5 . 


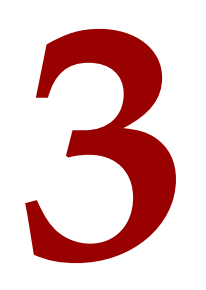

\section{Related Work}

After comprehensively identifying the requirements on deriving a simplistic dynamic model for ships, the state-of-the-art methods on the modeling of ship dynamics and the identification methods are worthy to be investigated to find pre-studies which are usable inspirations for this thesis. Hence, this chapter reviews the theory related to the ship dynamic model and system identification which are regarded as the foundations for the remainder of the thesis. Based on the classification of ship dynamics in Section 3.1.2 and detailed introduction to ship dynamic models in Section 3.1.3, three typical ship dynamic models containing the Abkowitz model, MMG model and vectorial representation are depicted concretely. Afterward, some basic concepts of system identification and its application to ship dynamics are presented in Section 3.2. The popular extensions of SVM, known as support vector classification algorithm (SVC) including the linear and nonlinear SVC in Section 3.2.3.1 and 3.2.3.2, are introduced respectively. Followed by the detailed description of LS-SVR in Section 3.2.3.4, the support vector regression algorithm (SVR) similar to SVC is presented in Section 3.2.3.3. 


\subsection{Ship Dynamic Model}

Ship dynamic model is defined to describe the ship's responses concerning the linear or angular velocities to forces and moments induced by commanded rudder angle and propeller shaft speed. The model used to abstractly describe ship dynamics and motions in cooperation with the kinematic model are built relevantly based on coordinate systems. As this topic has been studied for decades, uniform notations defined by the Society of Naval Architects and Marine Engineers (SNAME) are commonly adopted in the dynamic models in marine domain (SNAME 1950). To focus on the ship dynamic model, classifying ship dynamics clearly is necessary to present comprehensive insights. Afterward, understanding of ship dynamic models, for example, the Abkowitz model, MMG model, and vectorial model are easy to go.

\subsubsection{General Remarks: Reference Frames and Notations}

Reference Frames. To describe the motion of ships, reference frames are necessary to specify what the motion is relative to. Several reference frames are recommended for different research topics, e.g., the Earth Centered Inertial (ECI) frame for terrestrial navigation, Earth Centered Earth Fixed (ECEF) frame for global guidance, navigation and control, North-East-Down (NED) frame for flat earth navigation, body-fixed frame for describing velocities of body relative to the inertial reference frame, and flow axes for defining motion through a fluid (Fossen 2011).

In this thesis, the NED frame and body-fixed frame are selected to describe the motion of ships. The NED frame is also regarded as the earth-fixed frame in the flat earth navigation, which is defined relative to the Earth's reference ellipsoid (WGS-84). For the earth-fixed coordinate system, the axes $x, y$ and $z$ are usually defined as the $x$ axis directs to true north, the $y$ axis directs to the east, and $z$ axis directs downwards normal to earth's surface. In the earth-fixed frame, the first three coordinates and their time derivatives correspond to the position and translational motion along the $x$-axis, $y$-axis, and $z$-axis, while the last three coordinates and their time derivatives are used to describe the orientation and rotational motion (Fossen 2011). The body-fixed frame is a coordinate system moving along with the body. The origin center is always defined to coincide with a point midship in the water line. The axes $x_{b}, y_{b}$ and $z_{b}$ are usually set as the $x_{b}$ axis is towards the fore of ships, the $y_{b}$ axis directs to starboard of ships, and $z_{b}$ axis directs towards the bottom of ships. Finally, the two reference frames consisted of a body-fixed frame and an earth-fixed frame (regarded as the inertial frame for the use of Newton's law) are shown in Figure 3.1.1.

Planar Coordinate System. For some research issues of ships, f.i., control system designs, the 


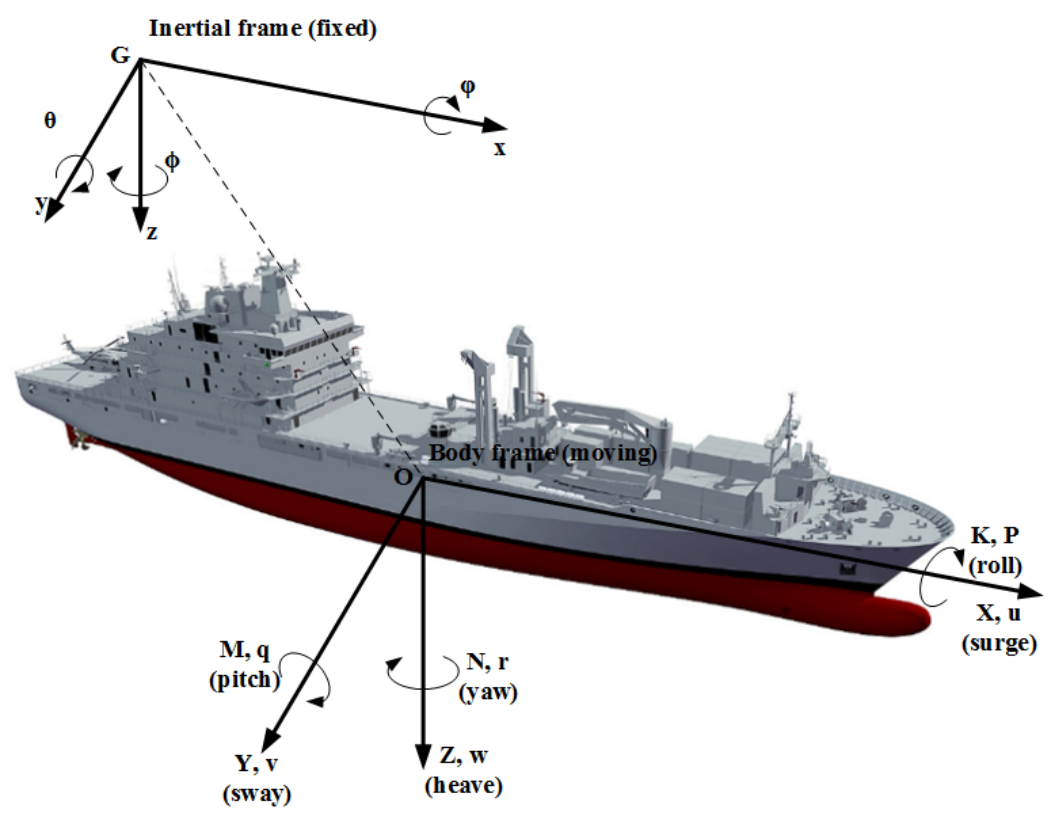

Figure 3.1.1: Illustration of reference frames.

surge, sway and yaw modes are mostly emphasized but the roll, heave and pitch modes are ignored. The corresponding planar coordinate system presented in Figure 3.1.2 is needed to describe the horizontal motions and obtained by removing the $z$ axis from the earth-fixed frame and the $z_{b}$ axis from the body-fixed frame.

Notations. The notations commonly-used to describe motions of ships are listed in Table 3.1.1. More information can be found in (SNAME 1950).

Table 3.1.1: Notations from SNAME.

\begin{tabular}{lllll}
\hline DOF & Motions & Forces & Linear velocity & Positions \\
\hline 1 & surge & $X$ & $u$ & $x$ \\
2 & sway & $Y$ & $v$ & $y$ \\
3 & heave & $Z$ & $w$ & $z$ \\
\hline & Rotations & Moments & Angular velocity & Rotation angles \\
\hline 4 & roll & $K$ & $p$ & $\phi$ \\
5 & pitch & $M$ & $q$ & $\theta$ \\
6 & yaw & $N$ & $r$ & $\psi$ \\
\hline
\end{tabular}




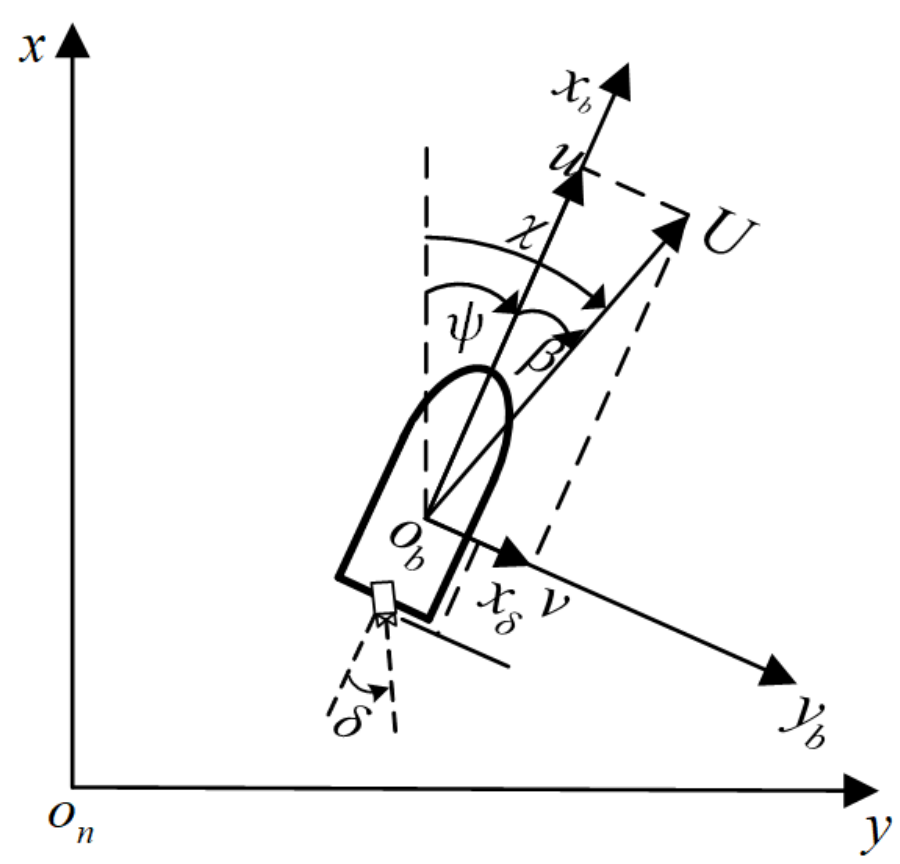

Figure 3.1.2: Description of planar motion variables. $x_{\delta}$ is the longitudinal moment arm from the center of rotation to the pivot point of the thruster/propulsion.

\subsubsection{Classification of Ship Dynamics}

The research of ship dynamics in general concerns two aspects, i.e., maneuvering (e.g., steering and maneuverability) and seakeeping (Pérez and Fossen 2006),(Fossen 2011). The maneuvering motions are generated by a ship moving at a constant positive speed in calm water in the absence of wave excitation. In the maneuvering studies, the horizontal motions (surge-sway-yaw) are commonly described by a 3 or 4 DOF model constructed based on the maneuvering theory. The focus of these studies is on the evaluation of ships' capability relevant to the action of control devices. The seakeeping motions refer to the motions of ships at zero or constant speed in waves, which can be analyzed using a seakeeping theory and modeled by a $6 \mathrm{DOF}$ model. The model can be used to evaluate ships' performance indices related to wave excitation. The unified calling of the combination of these two is 'maneuvering in seaway'.

Apart from the introduced classification way of ship dynamics, another useful approach on characterizing the dynamics of ships is to calculate the Froude number $F_{n}=\frac{U}{\sqrt{L g}}$ in which $L$ is the length of the submerged portion of the hull, $U$ is the operating speed of ships, and $g$ is the acceleration of gravity. Then the mode of ship dynamics can be defined according to the calculated number (Faltinsen 2005). For $F_{n}<0.4-0.5$, the hydrostatic force of buoyancy plays a prominent role on the operating ship, of which the condition is called displacement mode. In the 
planning mode with $F_{n}>1.0-1.2$, the hydrodynamic force dominates for carrying the weight of the ship. The hydrodynamic and hydrostatic forces are simultaneously working in carrying the weight of the ship in the semi-displacement mode for which $1.0-1.2<F_{n}<0.4-0.5$.

The overview of the classification of ship dynamics is graphically illustrated in Figure 3.1.3 (Pérez and Fossen 2006). Recall that the goal of this thesis is to find a solution for modeling dynamics for different types of ships, so a fact which should be noticed is that a ship may be designed as a special mode ship, but it routinely operates in each of the three modes. Additionally, the variations in ship performance could be observed if the mode of ship dynamics and corresponding Froude number were varying. Hence, the emphasizes should be on the described facts in this thesis. The effort on modeling dynamics for different types of ships should adequately capture the variations in ship performance over the range of achievable Froude numbers.

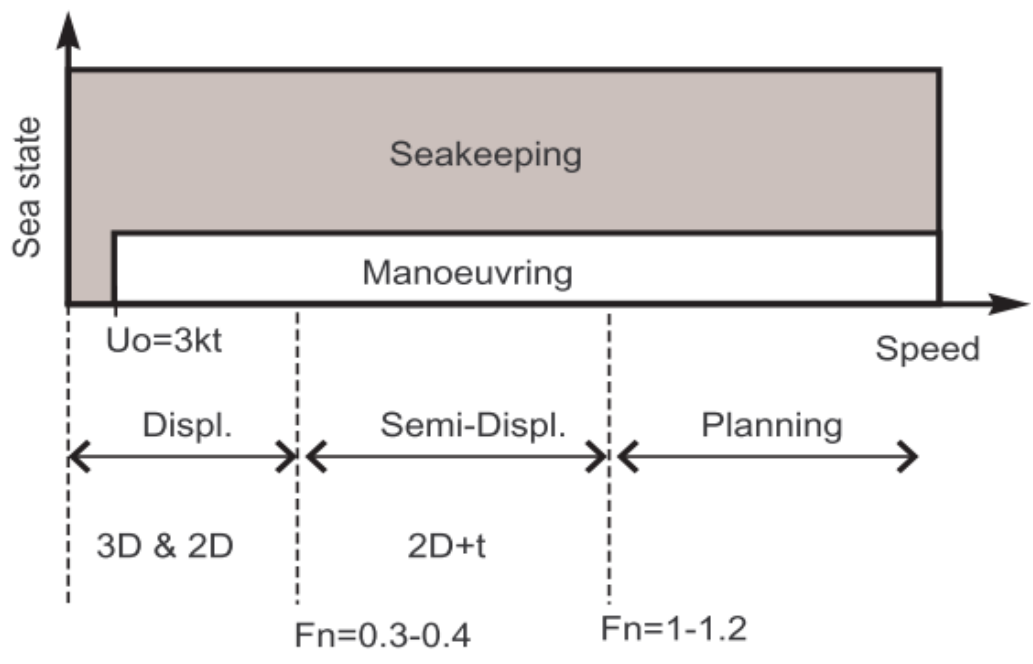

Figure 3.1.3: Illustration of the classification of ship dynamics (Pérez and Fossen 2006).

\subsubsection{Introduction to Ship Dynamic Models}

Since over one century ago, Davidson and Schiff (Davidson and Schiff 1964) started working with the mathematical modeling of ship dynamics. This model describes linear steering dynamics especially the interactions between sway and yaw. Based on this model, Nomoto (Nomoto, Taguchi, Honda, and Hirano 1957) derived a first or second order transfer function used for only analyzing yaw mode by eliminating the equation describing sway mode from the model proposed by Davison and Schiff. By considering the nonlinearity of ship dynamics in the operation of large 
rudder angle, Norrbin (Norrbin 1963) adjusted the form of Nomoto's first order transfer function by adding a nonlinear component. Analogously, Nomoto's second order transfer function was also modified by adding a nonlinear component by Bech and Wagner Smith (Bech and Smitt 1969), in which they additionally proposed the well-known Bech's reverse spiral maneuver and introduced the approach on applying such maneuver to estimate parameters in the nonlinear component. Either Nomoto's first or second linear transfer function or the corresponding modified first or second order nonlinear transfer function is still recognizable today and is still widely used for guidance and control design due to its simple and effective structure and easy-understanding.

A contribution to the work of modeling ship dynamics by Abkowitz (Abkowitz 1964)) should be highlighted. In his work, the forces (surge and sway) and moments (yaw) acting on a ship in 3 DOF are expressed by Taylor-series expansions at forward cruise speed and have an arbitrary degree of precision. The model involves a large number of coefficients, which further leads to a tough problem about how to estimate all these coefficients. Some works caring for this problem will be introduced later in the section on system identification of the ship dynamic model. Later, Son and Nomoto (Son and Nomoto 1982) expanded the application of the Abkowitz model to more ships, e.g., the large container ship, by analyzing and accounting the roll motions. As said previously, many coefficients are involved in the Taylor-series expansion models, and some terms have no physical interpretation, which leads to the estimation of coefficients difficult and furthermore makes the application of models troublesome. Therefore, simplifications to the models are meaningful as reported in some papers (Yeo and Rhee 2006) (Wang, Zou, Yang, and $\mathrm{Xu} 2015$ ) (Shenoi, Krishnankutty, and Selvam 2015) (Pérez and Blanke 2002), where the sensitivity study was commonly used to analyze how sensitive the model response to the variation of a model coefficient is. According to the results of the sensitivity study, the model was then simplified by omitting the coefficients with a very low sensitivity degree.

Different from the Abkowitz model, another new type model called modulus model was developed in 1963 by Fedayevsky and Sobolev (Fedyaevsky and Sobolev 1964) who used the quadratic modulus equations with low order to present hydrodynamic forces and moments. In 1970, Norrbin (Norrbin 1971) made some modifications of the former modulus model in modeling the hydrodynamic effects by using nonlinear quadratic and quartic terms for even functions and quadratic modulus terms for odd functions. This model is a little complicated for applications, so Blanke (Blanke 1981) simplified it by removing some unwieldy and unphysical terms at the same time maintaining the significant parts. To comprehensively study ship dynamics including roll motions, Christensen and Blanke (Christensen and Blanke 1986) 
developed a linear-time varying model with roll equation but without surge equation. Even though the surge equation is ignored, the forward speed generally resulted by the surge and sway speed is regarded as a varying parameter to be involved in sway, yaw and roll equations. Such a linear-time varying model is used to discuss the problems such as rudder roll damping problem and fin roll damping problem.

In the findings by Hooft (Hooft 1994), the cross-flow drag formulations were applied to analyze the lateral forces on a ship during maneuvering through tests performed as a function of the ship's lateral velocity and as a function of ship's yaw rate. The key advantage of cross-flow models is that it is dependent on a few hydrodynamic parameters, typically the drag coefficient for transverse flow and the hull friction coefficient (Simos, Pesce, Bernitsas, and Cohen 2002). With the development of such an approach within years, some studies make efforts to propose some comprehensive models, see the corresponding work found in (Beukelman and Journée 2001). Later, in the study on the maneuverability of ships, Toxopeus (Toxopeus 2011) developed a practical method that was incorporated with viscous-flow solvers used for predicting forces and moments on ships. This comprehensive method was investigated and indicated that it can improve the accuracy of maneuvering predictions.

For the modeling of ship dynamics, the work of the Mathematical Modeling Group makes the prominent influence and provides the fundaments on deriving models. In the work, the effects of hull hydrodynamic, propeller, rudder and their interactions are dealt with separately. The recommended references are (Inoue, Hirano, and Kijima 1981) (Kijima and Nakiri 1990) (Kijima, Katsuno, Nakiri, and Furukawa 1990).

The work by Fossen, especially presented in (Fossen 2011 ) and (Fossen 1994), demonstrates a comprehensive and extensive study of the latest research in navigation, guidance, control systems for marine vehicles. The detailed information about the implementation of these techniques is presented in a systematic way. The equations of motion are expressed in the form of matrix-vector, since nonlinear system properties such as symmetry, skew-symmetry, and positiveness of matrices can be exploited in the passivity or stability analysis, and such form of the model makes it easy to simulate the model in either Matlab or $\mathrm{C}++$ (Fossen 2013).

From the overview of the ship dynamic model, the works done by Nomoto, Abkowitz, MMG, and Fossen are the basis for many developed ship dynamic models. Therefore, the detailed presentation of these models will be given in the following. 


\subsubsection{Rigid-body Equations of Motion}

The rigid-body dynamics are derived by applying the first and second Euler axioms. In maritime domain, the ship is generally regarded as a rigid hull so that the motion equations are also suitable to study ship dynamics. The 6 DOF rigid-body equations of motion obtained are given when setting the center of gravity as $\left(x_{g}, y_{g}, z_{g}\right)$ and applying the SNAME notions (SNAME 1950).

$$
\begin{gathered}
m\left[\dot{u}-v r+w q-x_{g}\left(q^{2}+r^{2}\right)+y_{g}(p q-\dot{r})+z_{g}(p r+\dot{q})\right]=X \\
m\left[\dot{v}-w p+u r-y_{g}\left(r^{2}+p^{2}\right)+z_{g}(q r-\dot{p})+x_{g}(q p+\dot{r})\right]=Y \\
m\left[\dot{w}-u q+v p-z_{g}\left(p^{2}+q^{2}\right)+x_{g}(r p-\dot{q})+y_{g}(r q+\dot{p})\right]=Z \\
I_{x} \dot{p}+\left(I_{z}-I_{y}\right) q r-(\dot{r}+p q) I_{x z}+\left(r^{2}-q^{2}\right) I_{y z} \\
+(p r-\dot{q}) I_{x y}+m\left[y_{g}(\dot{w}-u q+v p)-z_{g}(\dot{v}-w p+u r)\right]=K \\
I_{y} \dot{q}+\left(I_{x}-I_{z}\right) r p-(\dot{p}+q r) I_{x y}+\left(p^{2}-r^{2}\right) I_{z x} \\
+(q p-\dot{r}) I_{y z}+m\left[z_{g}(\dot{u}-v r+w q)-x_{g}(\dot{w}-u q+v p)\right]=M \\
I_{z} \dot{r}+\left(I_{y}-I_{x}\right) p q-(\dot{q}+r p) I_{y z}+\left(q^{2}-p^{2}\right) I_{x y} \\
+(r q-\dot{p}) I_{z x}+m\left[x_{g}(\dot{v}-w p+u r)-y_{g}(\dot{u}-v r+w q)\right]=N
\end{gathered}
$$

where $X, Y, Z, K, M$ and $N$ are the external forces and moments, $I_{x}, I_{y}$ and $I_{z}$ are inertial moments about the $x_{g}, y_{g}$ and $z_{g}$ axes, $I_{x z}=I_{z x}, I_{y z}$ and $I_{x y}$ are the products of inertial.

For convenience, a set of basic assumptions are usually applied to simplify the 6 DOF equations of motion to 3 DOF because studies have indicated that the results from 3 DOF are mostly satisfactory for their research goals. These assumptions are given:

- The heave, roll and pitch modes are neglected.

- The distribution of ship mass is symmetrical with respect to the $O_{b}-x z$ plane.

- $I_{x y}=I_{y z}=0$.

- $y_{g}=$ o.

Then, the 3 DOF equations of motion in horizontal plane yields

$$
\begin{aligned}
m\left(\dot{u}-v r-x_{g} r^{2}\right) & =X \\
m\left(\dot{v}+u r+x_{g} \dot{r}\right) & =Y \\
I_{z} \dot{r}+m x_{g}(\dot{v}+u r) & =N
\end{aligned}
$$


As seen, this is the primary model for studying ship dynamics in 3 DOF. The left vital work is to define the external forces and moments acting on ships in the right-hand side terms of (3.2). These forces and moments can be classified into three kinds, i.e., radiation-induced forces and moments, propulsion forces and moments, and environmental forces and moments. The environmental forces and moments are discussed separately. The following sections will present three typical ways, i.e., Abkowitz's Taylor series expansion, modulus functions, and vectorial representations on describing the former two kinds of forces and moments. Focuses of this thesis are not on modeling environmental forces and moments. Interests in this part can refer to Chapter 8 of the handbook named Handbook of marine craft hydrodynamics and motion control (Fossen 2011).

\subsubsection{AвKowitz Model}

In Abkowitz model, the hydrodynamic forces and moments acting on the hull, propeller, and rudder are regarded as the function of state variables $(u, v, r)$ and actuator variable $(\delta)$, i.e., $f(u, v, r, \dot{u}, \dot{v}, \dot{r}, \delta)$, which is expanded by a 3 rd-order truncated Taylor-series at the steady state status given $u=u_{\mathrm{o}}, v=0$, and $r=0$. As the complexity of the 3 rd-order Taylor series expansion is very high due to its large number of terms, several pieces of good assumptions proposed by Abkowitz are used to simplify the expansions. These assumptions are given

- A 3 rd-order truncated Taylor series expansion for the steady state at $u=u_{\circ}$ can be used to describe mostly ship maneuvers.

- Only 1st-order acceleration terms are taken into consideration.

- The coupling between the acceleration and velocity terms is ignorable.

- Standard port/starboard symmetry simplifications except terms describing the constant force and moment arising from single-screw propellers. 
After applying these assumptions, the force and moment terms in $(3.2)$ can be defined as

$$
\begin{aligned}
X= & X^{*}+X_{\dot{u}} \Delta \dot{u}+X_{u} \Delta u+X_{u u} \Delta u^{2}+X_{u u u} \Delta u^{3}+X_{v v} v^{2}+X_{r r} r^{2} \\
& +X_{\delta \delta} \delta^{2}+X_{r v} r v+X_{r \delta} r \delta+X_{v \delta} \delta \delta+X_{u v v} \Delta u v^{2}+X_{u r r} \delta u r^{2} \\
& +X_{u \delta \delta} \Delta u \delta^{2}+X_{u v r} \Delta u v r+X_{u r \delta} \Delta u r \delta+X_{u v \delta} \Delta u v \delta \\
Y= & Y^{*}+Y_{u} \Delta u+Y_{u u} \Delta u^{2}+Y_{r} r+Y_{v} v+Y_{r} \dot{r}+Y_{v} \dot{v}+Y_{\delta} \delta+Y_{r r r} r^{3} \\
& +Y_{v v v} v^{3}+Y_{\delta \delta \delta} \delta^{3}+Y_{r r \delta} r^{2} \delta+Y_{r \delta \delta} r \delta^{2}+Y_{r r v} r^{2} v+Y_{v v r} v^{2} r \\
& +Y_{v \delta \delta} v \delta^{2}+Y_{v v \delta} v^{2} \delta+Y_{v r \delta} v r \delta+Y_{u v} \Delta u v+Y_{u u v} \Delta u^{2} v+Y_{u r} \Delta u r \\
& +Y_{u u r} \Delta u^{2} r+Y_{u \delta} \Delta u \delta+Y_{u u \delta} \Delta u^{2} \delta \\
N= & N^{*}+N_{u} \Delta u+N_{u u} \Delta u^{2}+N_{r} r+N_{v} v+N_{r} \dot{r}+N_{v} \dot{v} \\
& +N_{\delta} \delta+N_{r r r} r^{3}+N_{v v v} v^{3}+N_{\delta \delta \delta} \delta^{3}+N_{r r \delta} r^{2} \delta+N_{r \delta \delta} r \delta^{2}+N_{r r v} r^{2} r \\
& +N_{v v r} v^{2} r+N_{v \delta \delta} v \delta^{2}+N_{v v \delta} v^{2} \delta+N_{v r \delta} v r \delta+N_{u v} \Delta u v+N_{u u v} \Delta u^{2} v \\
& +N_{u r} \Delta u r+N_{u u r} \Delta u^{2} r+N_{u \delta} \Delta u \delta+N_{u u \delta} \Delta u^{2} \delta
\end{aligned}
$$

where $\Delta u=u-u_{0}, v=\Delta v, r=\Delta r, \delta=\Delta \delta, \Delta \dot{u}=\dot{u}, \dot{v}=\Delta \dot{v}, \dot{r}=\Delta \dot{r} ; X_{\dot{u}}, Y_{\dot{v}}, Y_{\dot{r}}, N_{\dot{r}}$, and $N_{\dot{v}}$ are partial derivatives of hydrodynamic acceleration and angular acceleration.

In Abkowitz's model, the forces and moments are expressed by a function of various factors and expanded regarding the Taylor series. From a theoretical point of view, this model is relatively complete. The ship hull, propeller, and rudder are assumed as a whole system such that the complex interference between them doesn't need to analyze and consider, but it is inconvenient to make local changes of the design of the ship hull, propeller or rudder. The model contains dozens of hydrodynamic coefficients, which is a huge task to estimate these coefficients. Moreover, the physical interpretation of some coefficients is not clear.

\subsubsection{MMG MODEL}

MMG model emphasizes the physical interpretation of each hydrodynamic coefficient and excludes some meaningless and unimportant coefficients indicated by the captive model test. Additionally, the interference between the hull of the ship, propeller, and rudder is accounted for. The coefficients in the MMG model can be estimated by the captive model test. Supposing the 
starboard/port symmetry gives the 3 DOF MMG model as

$$
\begin{aligned}
\left(m-X_{\dot{u}}\right) \dot{u}-\left(m-Y_{\dot{v}}\right) v r-m x_{g} r^{2} & =X_{H}+X_{P}+X_{R} \\
\left(m-Y_{\dot{v}}\right) \dot{v}-\left(m-X_{\dot{u}}\right) u r+m x_{g} \dot{r} & =Y_{H}+Y_{P}+Y_{R} \\
\left(I_{z}-N_{\dot{r}}\right) \dot{r}+m x_{g}(\dot{v}+u r) & =N_{H}+N_{P}+N_{R}
\end{aligned}
$$

where $X_{H}, Y_{H}$ and $N_{H}$ donate the hull viscous forces and moment, respectively. $X_{P}, Y_{P}$ and $N_{P}$ are propulsive (propeller, thrusters, etc.) forces and moment, respectively. $X_{R}, Y_{R}$ and $N_{R}$ present rudder forces and moment, respectively.

For deciding the hull forces and moment, several methods can be used, e.g., the Taylor-series expansion, the model proposed by Inoue et al. (Inoue et al. 1981), and approximate formulae obtained by regression investigation for 15 kinds of ships and their 48 loading conditions (Kijima 2002). More detailed information can refer to the mentioned papers.

Propulsive forces and moment are modeled by

$$
\begin{aligned}
X_{P} & =\left(1-t_{P}\right) \rho n^{2} D_{P}^{4} k_{T} \\
Y_{P} & =0 \\
N_{P} & =0
\end{aligned}
$$

in which $t_{P}$ is the propeller thrust deduction coefficient, $n$ is the propeller shaft speed, $D_{P}$ presents propeller diameter, $k_{T}$ donates the propeller thrust coefficient which is usually calculated by the linear regression formula or parabolic formula. The linear formula is given as

$$
k_{T}=a_{1} J+a_{\circ}=\frac{a_{1} u_{a}}{n D_{P}}+a_{\circ}
$$

where $J$ is the advance coefficient, $\alpha_{\circ}$ and $\alpha_{1}$ are constant coefficients which can be estimated by the open-water propeller test, $u_{a}$ means the advance speed of the propeller.

The models for rudder forces and moment are governed by

$$
\begin{aligned}
X_{R} & =\left(1-t_{R}\right) F_{N} \sin \delta \\
Y_{R} & =\left(1+a_{H}\right) F_{N} \cos \delta \\
N_{R} & =\left(x_{R}+a_{H} x_{H}\right) F_{N} \cos \delta
\end{aligned}
$$

where $t_{R}$ is the rudder thrust deduction coefficient, $a_{H}$ is the correction factor of steering induced hull lateral force, $x_{R}$ is the longitudinal moment arm from the center of rudder force to the origin 
center of the body-fixed frame, $x_{H}$ is the longitudinal moment arm from the center of steering induced lateral force to the origin center of the body-fixed frame, $F_{N}$ is the rudder normal force which is expressed as

$$
F_{N}=-\frac{6.13 \Lambda}{\Lambda+2.25} \cdot \frac{A_{R}}{L^{2}}\left(u_{R}^{2}+v_{R}^{2}\right) \sin a_{R}
$$

where $\Lambda$ is the rudder aspect ratio, $A_{R}$ is the rudder area, $a_{R}$ is the effective angle induced by the fluid to the rudder, $u_{R}$ and $v_{R}$ are the normal and tangential velocity induced by fluid at the rudder, respectively.

Comparing with Abkowitz's model, one can conclude that

- Each term in the MMG model has its manifest physical interpretation.

- The value of each coefficient can be estimated through experiments.

- The model obtained for the scale-model ship can be converted effectively to match the real corresponding ship, that means the scale effects caused by the discrepancy of Reynold number between the scale-model ship and real ship make an ignorable influence on the application of the obtained model for the scale-model ship to real ship.

- It is convenient to make changes on the hull, rudder or propeller because each of them and interference between them are modeling separately.

\subsubsection{Vectorial RePRESENTATions}

In this part, the ship dynamic model proposed in the form of vectorial representations is comprehensively elaborated from the nonlinear $6 \mathrm{DOF}$ vectorial representations. The kinematic (3.9) and nonlinear 6 DOF dynamic model (3.10) of a ship can be expressed in the body-fixed frame, respectively (Fossen 1994).

$$
\dot{\eta}=J(\eta) v
$$

where $v=[u, v, w, p, q, r]^{T}$ is the spatial velocity state vector, $\eta=[x, y, z, \phi, \theta, \psi]^{T}$ presents the position and orientation states, $J(\eta)=f(\phi, \theta, \psi)$ is the transformation matrix between variants in the body-fixed frame and earth-fixed frame, which yields in Euler-angle transformation form as 
follows with abridged notations including $c()=.\cos (),. s()=.\sin (),. t()=.\tan ($.$) .$

$$
J(\eta)=\left[\begin{array}{cccccc}
c \phi c \theta & (c \phi s \theta s \varphi-s \phi c \varphi) & (s \phi s \varphi+c \phi c \phi s \theta) & 0 & 0 & 0 \\
s \phi c \theta & (c \phi c \phi+s \phi s \theta s \phi) & (s \theta s \phi c \varphi-c \phi s \varphi) & 0 & 0 & 0 \\
-s \theta & c \theta s \varphi & c \theta c \phi & 0 & 0 & 0 \\
0 & 0 & 0 & 1 & t \theta s \varphi & t \theta c \varphi \\
0 & 0 & 0 & 0 & c \varphi & -s \varphi \\
0 & 0 & 0 & 0 & \frac{s \varphi}{c \theta} & \frac{c \varphi}{c \theta}
\end{array}\right]
$$

A set of equations in (3.1) are converted into the form of vectorial representations by introducing some modifications by Fossen et al., which leads to

$$
M \dot{v}+C(v) v+D(v) v+g(\eta)=\tau_{\text {ext }}+\tau
$$

where the mass matrix $M$ is the summation of the added mass $M_{A}$ and the rigid body mass $M_{R B}$, $C(v)$ is the Coriolis and centripetal matrix due to the rotation of body-fixed frame with respect to the inertial frame, $D(v)$ is the damping matrix, $g(\eta)$ manifests the effects of buoyancy's interaction with gravity, $\tau=\left[\tau_{X}, \tau_{Y}, \tau_{Z}, \tau_{K}, \tau_{M}, \tau_{N}\right]^{T}$ denotes the actuator forces and moments generated by a set of propellers with revolutions per second $n=\left[n_{1}, n_{2}, \cdots, n_{P_{1}}\right]^{T}$ and a set of control surfaces with angles $\delta=\left[\delta_{1}, \delta_{2}, \cdots, \delta_{P_{2}}\right]^{T}, \tau_{\text {ext }}$ is the external disturbances induced by currents, wave, etc. Details about $M, C(v), D(v)$ and $g(\eta)$ are carefully explained in the following.

$$
M=M_{R B}+M_{A}=\left[\begin{array}{cccccc}
m-X_{\dot{u}} & -X_{\dot{v}} & -X_{\dot{w}} & -X_{\dot{p}} & m z_{g}-X_{\dot{q}} & -m y_{g}-X_{\dot{r}} \\
-Y_{\dot{u}} & m-Y_{\dot{v}} & -Y_{\dot{w}} & -m z_{g}-Y_{\dot{p}} & -Y_{\dot{q}} & m x_{g}-Y_{\dot{r}} \\
-Z_{\dot{u}} & -Z_{\dot{v}} & m-Z_{\dot{w}} & m y_{g}-Z_{\dot{p}} & -m x_{g}-Z_{\dot{q}} & -Z_{\dot{r}} \\
-K_{\dot{u}} & -m z_{g}-K_{\dot{v}} & m y_{g}-K_{\dot{w}} & I_{x}-K_{\dot{p}} & -I_{x y}-K_{\dot{q}} & -I_{x z}-K_{\dot{r}} \\
m z_{g}-M_{\dot{u}} & -M_{\dot{v}} & -m x_{g}-M_{\dot{w}} & -I_{y x}-M_{\dot{p}} & I_{y}-M_{\dot{q}} & -I_{y z}-M_{\dot{r}} \\
-m y_{g}-N_{\dot{u}} & m x_{g}-N_{\dot{v}} & -N_{\dot{w}} & -I_{z x}-N_{\dot{p}} & -I_{z y}-N_{\dot{q}} & I_{z}-N_{\dot{r}}
\end{array}\right]
$$

in which

$$
M_{R B}=\left[\begin{array}{cccccc}
m & 0 & 0 & 0 & m z_{g} & -m y_{g} \\
0 & m & 0 & -m z_{g} & 0 & m x_{g} \\
0 & 0 & m & m y_{g} & -m x_{g} & 0 \\
0 & -m z_{g} & m y_{g} & I_{x} & -I_{x y} & -I_{x z} \\
m z_{g} & 0 & -m x_{g} & -y_{y x} & I_{y} & -I_{y z} \\
-m y_{g} & m x_{g} & 0 & -I_{z x} & -I_{z y} & I_{z}
\end{array}\right],
$$




$$
M_{A}=-\left[\begin{array}{cccccc}
X_{\dot{u}} & X_{\dot{v}} & X_{\dot{w}} & X_{\dot{p}} & X_{\dot{q}} & X_{\dot{r}} \\
Y_{\dot{u}} & Y_{\dot{v}} & Y_{\dot{w}} & Y_{\dot{p}} & Y_{\dot{q}} & Y_{\dot{r}} \\
Z_{\dot{u}} & Z_{\dot{v}} & Z_{\dot{w}} & Z_{\dot{p}} & Z_{\dot{q}} & Z_{\dot{r}} \\
K_{\dot{u}} & K_{\dot{v}} & K_{\dot{w}} & K_{\dot{p}} & K_{\dot{q}} & K_{\dot{r}} \\
M_{\dot{u}} & M_{\dot{v}} & M_{\dot{w}} & M_{\dot{p}} & M_{\dot{q}} & M_{\dot{r}} \\
N_{\dot{u}} & N_{\dot{v}} & N_{\dot{w}} & N_{\dot{p}} & N_{\dot{q}} & N_{\dot{r}}
\end{array}\right],
$$

$m$ is the mass of the rigid body, $I_{x}, I_{y}$, and $I_{z}$ are the inertial moments with respect to the $x$-axis, $y$-axis, and $z$-axis in the body-fixed frame, $I_{x y}=I_{y x}, I_{x z}=I_{z x}$, and $I_{y z}=I_{z y}$ are the inertial products. Meanwhile, the inertial matrix of gravity center can be defined as

$$
I_{g}=\left[\begin{array}{ccc}
I_{x} & -I_{x y} & -I_{x z} \\
-I_{y x} & I_{y} & -I_{y z} \\
-I_{z x} & -I_{z y} & I_{z}
\end{array}\right] .
$$

From the notations of SNAME, the hydrodynamic added mass, for instance, $Y$ along with the $y$-axis of the body-fixed frame due to an acceleration $\dot{u}$ in $x$ direction can be written as

$$
Y=-Y_{\dot{u}} \dot{u}, \quad Y_{\dot{u}}=\frac{\partial Y}{\partial \dot{u}}
$$

The rest hydrodynamic terms in $M_{A}$ are expressed similarly.

$$
\begin{aligned}
& C(v)=C_{R B}(v)+C_{A}(v) \\
& \begin{array}{c}
{\left[\begin{array}{ccc}
0 & 0 & 0 \\
0 & 0 & 0 \\
0 & 0 & \circ \\
-m\left(y_{g} q+z_{g} r\right) & m\left(y_{g} p+w\right)-a_{3} & m\left(z_{g} p-v\right)+a_{2} \\
m\left(x_{g} q-w\right)+a_{3} & -m\left(z_{g} r+x_{g} p\right) & m\left(z_{g} q+u\right)-a_{1} \\
m\left(x_{g} r+v\right)-a_{2} & m\left(y_{g} r-u\right)+a_{1} & -m\left(x_{g} p+x_{g} q\right) \\
= & -m\left(x_{g} q-w\right)-a_{3} & -m\left(x_{g} r+v\right)+a_{2} \\
-m\left(y_{g} q+z_{g} r\right) & m\left(z_{g} r+x_{g} p\right) & -m\left(y_{g} r-u\right)-a_{1} \\
-m\left(y_{g} p+w\right)+a_{3} & -m\left(z_{g} q+u\right)+a_{1} & m\left(x_{g} p+x_{g} q\right) \\
\circ & -a_{y z} q-I_{x z} p+I_{z} r-b_{3} & I_{y z} r+I_{x y} p-I_{y} q+b_{2} \\
I_{y z} q+I_{x z} p-I_{z} r+b_{3} & \circ & -I_{x z} r-I_{x y} q+I_{x} p-b_{1} \\
-I_{y z} r-I_{x y} p+I_{y} q-b_{2} & I_{x z} r+I_{x y} q-I_{x} p+b_{1} & \circ
\end{array}\right]}
\end{array}
\end{aligned}
$$

where the $a_{i}(i=1,2,3)$ and $b_{j}(j=1,2,3)$ donating terms of $C_{A}(v)$ can be derived by the energy 
formulation $T_{A}$ written as a quadratic form based on the $M_{A}$.

$$
T_{A}=\frac{1}{2} v^{T} M_{A} v, \quad \dot{M}_{A}=0
$$

Suppose the fluid is incompressible, then the Kirchhoff's equations for a rigid body gives

$$
\begin{array}{r}
\frac{d}{d t}\left(\frac{\partial T_{A}}{\partial v_{1}}\right)+S\left(v_{2}\right) \frac{\partial T_{A}}{\partial v_{1}}=\tau_{1} \\
\frac{d}{d t}\left(\frac{\partial T_{A}}{\partial v_{2}}\right)+S\left(v_{2}\right) \frac{\partial T_{A}}{\partial v_{2}}+S\left(v_{1}\right) \frac{\partial T_{A}}{\partial v_{1}}=\tau_{2}
\end{array}
$$

where $\tau_{1}=\left[X_{A}, Y_{A}, Z_{A}\right]^{T}$ and $\tau_{2}=\left[K_{A}, M_{A}, N_{A}\right]^{T}$ are the added mass terms. The skew-symmetric cross-product operations $S\left(v_{1}\right)$ and $S\left(v_{2}\right)$ are defined respectively as

$$
S\left(v_{1}\right)=-S^{T}\left(v_{1}\right)=\left[\begin{array}{ccc}
0 & -w & v \\
w & 0 & -u \\
-v & u & 0
\end{array}\right], S\left(v_{2}\right)=-S^{T}\left(v_{2}\right)=\left[\begin{array}{ccc}
0 & -r & q \\
r & 0 & -p \\
-q & p & 0
\end{array}\right]
$$

Subsequently, the expression of $C_{A}(v)$ extracted from the above procedure is

$$
C_{A}(v)=\left[\begin{array}{cccccc}
0 & 0 & 0 & 0 & -a_{3} & a_{2} \\
0 & 0 & 0 & a_{3} & 0 & -a_{1} \\
0 & 0 & 0 & -a_{2} & a_{1} & 0 \\
0 & a_{3} & a_{2} & 0 & -b_{3} & b_{2} \\
a_{3} & 0 & -a_{1} & a_{3} & 0 & -b_{1} \\
-a_{2} & a_{1} & 0 & -b_{2} & b_{1} & 0
\end{array}\right]
$$

with

$$
\left\{\begin{array}{cc}
a_{1}= & X_{\dot{u}} u_{r}+X_{\dot{v}} v_{r}+X_{\dot{w}} w_{r}+X_{\dot{p}} p+X_{\dot{q}} q+X_{\dot{r}} r \\
a_{2}= & Y_{\dot{u}} u_{r}+Y_{\dot{v}} v_{r}+Y_{\dot{w}} w_{r}+Y_{\dot{p}} p+Y_{\dot{q}} q+Y_{\dot{r}} r \\
a_{3}= & Z_{\dot{u}} u_{r}+Z_{\dot{v}} v_{r}+Z_{\dot{w}} w_{r}+Z_{\dot{p}} p+Z_{\dot{q}} q+Z_{\dot{r}} r \\
b_{1}= & K_{\dot{u}} u_{r}+K_{\dot{v}} v_{r}+K_{\dot{w}} w_{r}+K_{\dot{p}} p+K_{\dot{q}} q+K_{\dot{r}} r \\
b_{2}= & M_{\dot{u}} u_{r}+M_{\dot{v}} v_{r}+M_{\dot{w}} w_{r}+M_{\dot{p}} p+M_{\dot{q}} q+M_{\dot{r}} r \\
b_{3}= & N_{\dot{u}} u_{r}+N_{\dot{v}} v_{r}+N_{\dot{w}} w_{r}+N_{\dot{p}} p+N_{\dot{q}} q+N_{\dot{r}} r
\end{array} .\right.
$$

The damping effect due to complicated hydrodynamic phenomena is the most uncertain 
component and crucial to be decided. But in general, it is always composed of the linear damping component and nonlinear quadratic damping component such that

$$
D(v)=D_{L}+D_{N L}(v)
$$

with

$$
\begin{aligned}
& D_{L}=-\left[\begin{array}{cccccc}
X_{u} & X_{v} & X_{w} & X_{p} & X_{q} & X_{r} \\
Y_{u} & Y_{v} & Y_{w} & Y_{p} & Y_{q} & Y_{r} \\
Z_{u} & Z_{v} & Z_{w} & Z_{p} & Z_{q} & Z_{r} \\
K_{u} & K_{v} & K_{w} & K_{p} & K_{q} & K_{r} \\
M_{u} & M_{v} & M_{w} & M_{p} & M_{q} & M_{r} \\
N_{u} & N_{v} & N_{w} & N_{p} & N_{q} & N_{r}
\end{array}\right], \\
& D_{N L}(v)=-\left[\begin{array}{cccccc}
X_{|u| u}|u| & X_{|v| v}|v| & X_{|w| w}|w| & X_{|p| p}|p| & X_{|q| q}|q| & X_{|r| r}|r| \\
Y_{|u| u}|u| & Y_{|v| v}|v|+Y_{|r| v}|r| & Y_{|w| w}|w| & Y_{|p| p}|p| & Y_{|q| q}|q| & Y_{|r| r}|r|+Y_{|v| r}|v| \\
Z_{|u| u}|u| & Z_{|v| v}|v| & Z_{|w| w}|w| & Z_{|p| p}|p| & Z_{|q| q}|q| & Z_{|r| r}|r| \\
K_{|u| u}|u| & K_{|v| v}|v| & K_{|w| w \mid}|w| & K_{|p| p}|p| & K_{|q| q}|q| & K_{|r| r}|r| \\
M_{|u| u}|u| & M_{|v| v}|v| & M_{|w| w}|w| & M_{|p| p}|p| & M_{|q| q}|q| & M_{|r| r}|r| \\
N_{|u| u}|u| & N_{|v| v}|v|+N_{|r| v}|r| & N_{|w| w}|w| & N_{|p| p}|p| & N_{|q| q}|q| & N_{|r| r}|r|+N_{|v| r} v
\end{array}\right]
\end{aligned}
$$

where some additional nonlinear coupling terms are involved on the diagonal in heave, roll, and pitch, which is because only terms among all additional coupling terms in 6 DOF affect obviously are retained.

The formulae of $g(\eta)$, effects of buoyancy's interaction with gravity, is given as

$$
g(\eta)=-\left[\begin{array}{c}
-\rho g \int_{0}^{z} A_{w p}(\zeta) d \zeta \sin \theta \\
\rho g \int_{0}^{z} A_{w p}(\zeta) d \zeta \cos \theta \sin \varphi \\
\rho g \int_{0}^{z} A_{w p}(\zeta) d \zeta \cos \theta \cos \varphi \\
\rho g \nabla G \bar{M}_{T} \cos \theta \sin \varphi \cos \varphi \\
\rho g \nabla G \bar{M}_{L} \sin \theta \cos \theta \cos \varphi \\
\rho g \nabla\left(-\overline{G M}_{L} \cos \theta+\overline{G M}_{T}\right) \sin \varphi \sin \theta
\end{array}\right]
$$

where $\rho$ is the water density, $g$ is the gravity acceleration, $z$ is the depth at the lowest point of the ship, $\nabla$ is the displaced water volume, $A_{w p}(\zeta)$ is the waterplane area, $\overline{G M}_{T}$ is the transverse metacentric height, $\overline{G M}_{L}$ is the longitudinal metacentric height. 


\subsubsection{Requirement Fulfillment Summary}

The motions of the surge, sway, yaw, pitch, roll, and heave can be described with the use of six differential equations and body-fixed and earth-fixed frames once the ship is regarded as a rigid-body object. In these differential equations, the terms with the meaning of external forces and moments acting on ships containing radiation-induced and propulsion forces and moments are able to be respectively defined by means of Abkowitz's Taylor series expansion, modulus functions, and vectorial representations. The following gives a comprehensive overview of these three models, including two folds, i.e., merits and demerits.

- In Abkowitz's model, the hull, propeller, and rudder of the ship are assumed as a holistic system, for which the relatively induced forces and moments are completely expressed by the Taylor series. But its application is restricted due to three facts. The local variation in the design of ship hull, propeller or rudder and the analysis of their interactions are impossible. Some coefficients have no clear physical interpretation. Too many coefficients involved in the model make the multicollinearity highly happen, which meanwhile challenges the estimation of coefficients. Hence, one can see that only the fourth requirement is fulfilled.

- In MMG model, external forces and moments generated by ship hull, propeller and rudder are represented separately by modulus functions but completely captured, which makes each term have manifest physical interpretation at the same time makes changes on hull, propeller or rudder possible. The coefficients can be estimated through a suitable method necessarily incorporated with optimal experiments, but it is cumbersome and high finance-consuming to estimate so many coefficients. What's more, it is not to ensure convenient and fast model simulations. So, this model fulfills the first and fourth requirements and partially fulfills the third requirement.

- In the vectorial representation model, the equations of motion are completely expressed in the form of matrix-vector where each term can be interpreted physically and adjusted according to the property variations of hull, propeller or rudder. Applying the model for simulation is easy to be conducted in either Matlab or $\mathrm{C}++$. The irreplaceable advantage of this model is that the nonlinear system properties such as symmetry, skew-symmetry, and positiveness of matrices can be exploited in the passivity or stability analysis. Unavoidably, it must stand the pressure on demand for cumbersome processes and high financial consumption in estimating all coefficients. Therefore, it is indicated that this model fulfills 
the first and fourth requirements and partially fulfills the third requirement.

One remark can be concluded that the derived requirements on ship dynamic model are not fully fulfilled.

\subsection{System IDENTIFicAtion}

System identification is a subcategory of estimation in the system theory, which is the process of developing a mathematical representation of a physical system from experimental data by using statistical methods. In applications, modeling based on system identification is accomplished through the procedure starting from generating and processing observations until strongly validating the identified model with relatively acceptable accuracy, which is also followed to model ship dynamics. Through the overview of the identification of ship dynamic models, the support vector machines based identification method is highlighted due to comparatively attractive advantages.

\subsubsection{Procedure}

Generally, the system identification procedure is a natural logical flow shown in Figure 3.2.1 (Ljung 1998) where four core steps are illustrated and further depicted concretely as follows.

\subsubsection{The Observed Data}

The data are collected from the optimal experiment which is designed sometimes based on the prior knowledge so that the recorded data are maximally informative. As suggested by Ljung (Ljung 1998), the input used to generate training maneuvers to supply sufficiently informative data for identification should satisfy the persistence of excitation condition for guaranteeing convergence and ensuring robustness properties of identification method. Ideally, the phase trajectories resulting from such maneuvers are ought to fill some sufficiently large domain in the state space. For instance, the pseudo-random binary sequences (PRBS) are rich enough and desirable choice to guarantee reliable estimation of all parameters involved in the model (Sutulo and Soares 2014). But PRBS requires very long tests to achieve good loop filling. What's more, the desirable maneuvers are in fact not possible to be achieved and not simple to be executed with the standard means of control. From the studies many researchers have done on 


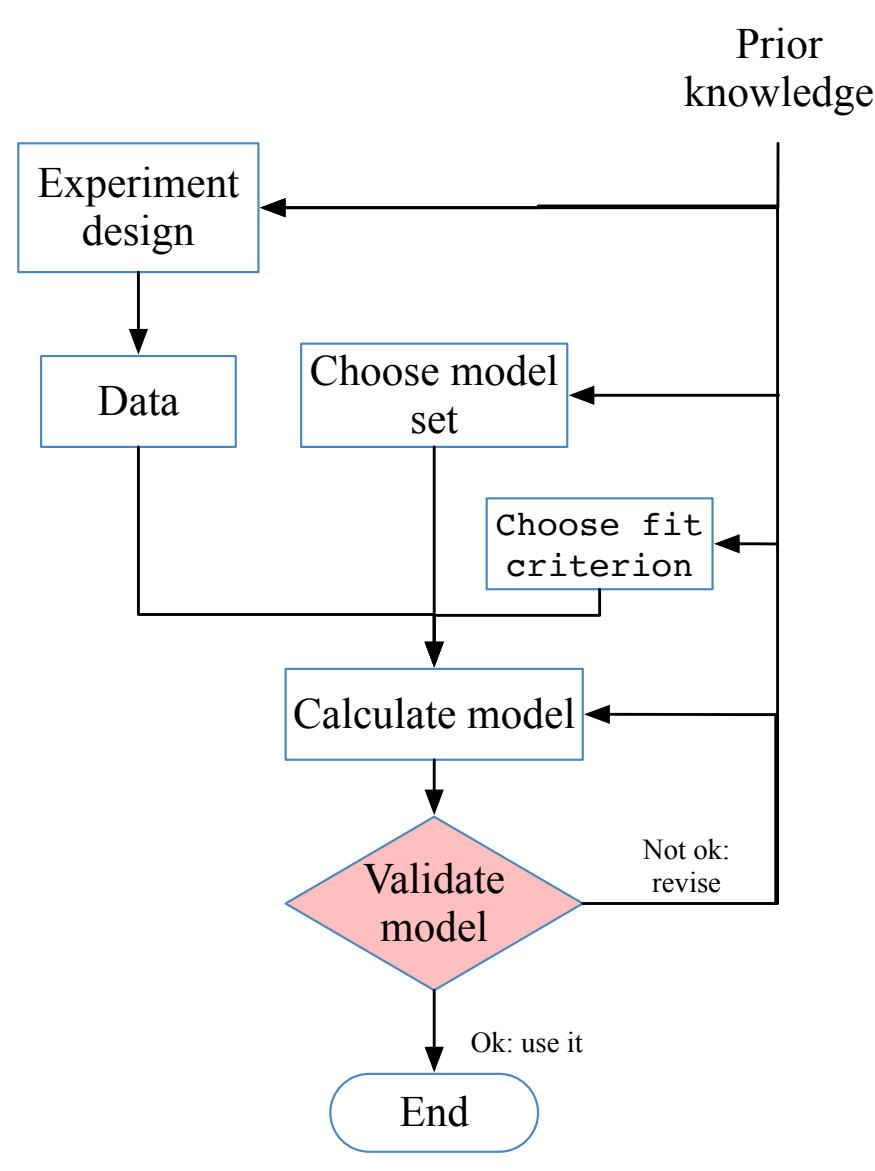

Figure 3.2.1: The system identification procedure.

the identification of ship mathematical models, the standard zigzag maneuvers can be alternatives (Yoon and Rhee 2003) (Sutulo and Soares 2014).

\subsubsection{The Set of Models or the Model Structure}

This step is usually the most challenging part of the identification procedure. The best suitable model needs to be selected from a set of models or model structures by specifying within these models. Models are generally categorized into black-box models and gray-box models. The black-box model donates the model whose parameters are basically viewed as vehicles for adjusting the fit to the data and do not reflect physical considerations in the system (Ljung 1998). The gray-box model refers to the model with adjustable parameters with physical interpretation (Ljung 1998). In the maritime domain, such model sets for describing ship dynamics can be built based on the above-introduced kinds of ship dynamic models. In other words, the models can also be called gray-box models. 


\subsubsection{IDENTIFICATION Method}

The identification method is responsible for searching and determining the best model within the set. The quality of the model is decided by assessing the performance of the model on reproducing the measured data. The determination of the identification method is dependent on the model structure used. For a model structure, sometimes not only one method is useful. Therefore, the identification methods used to identify ship models will be introduced in detail in Section 3.2.2 for the selection of a method suitable for this thesis.

\subsubsection{Model Validation}

After the previous three steps, the best model with a particular structure can be obtained but not determined yet because it remains to be tested whether it is good enough for the intended purpose, i.e., model validation. The validation can be executed by analyzing and comparing the predictions of the identified model and measured data that have not been used for identification.

\subsubsection{Identification of The Ship Dynamic Model}

The application of system identification to identify the ship dynamic model can be briefly described. The parameters of the ship dynamic model are estimated which is guided by a hypothesized model structure and the collected observed data set. If the validation test on the identified model is ideal, the identified dynamic model is feasible to describe and capture ship dynamics. As the identification method for this thesis has not determined yet, the following will present related details about the use of the identification method in the maritime domain to decide which method is suitable for this study.

Basically, the framework of the identification of the ship dynamic model according to the system identification procedure is illustrated in Figure 3.2.2. For the identification method, many choices exist for performing identification based modeling of ship dynamics. Åström and Källström (Åström and Källström 1976) estimated the parameters of the ship steering model by applying the measurements from full-scale tests to the maximum likelihood method (ML). Using the experimental data of the Esso Osaka tanker, Hwang (Hwang 1980) applied the Extended Kalman Filtering algorithm (EKF) to solve the estimation of Abkowitz model parameters and modified the model on the basis of the identification results. The model of the Esso Osaka tanker was also identified using the ridge regression method by Yoon and Rhee (Yoon and Rhee 2003). In Taylor's work (Taylor 2000), EKF was applied to estimate coefficients of a dynamic model for an Arleigh Burke Class Destroyer for the model-based control design. Caccia, et al. (Caccia, 
Bruzzone, and Bono 2008) used LS and data stemmed from sea trails to estimate parameters of the proposed practical dynamic model for an autonomous catamaran prototype Charlie2005. Additionally, the modified or improved LS is also studied to work in identifying ship dynamic models, see (Pullard 2003).

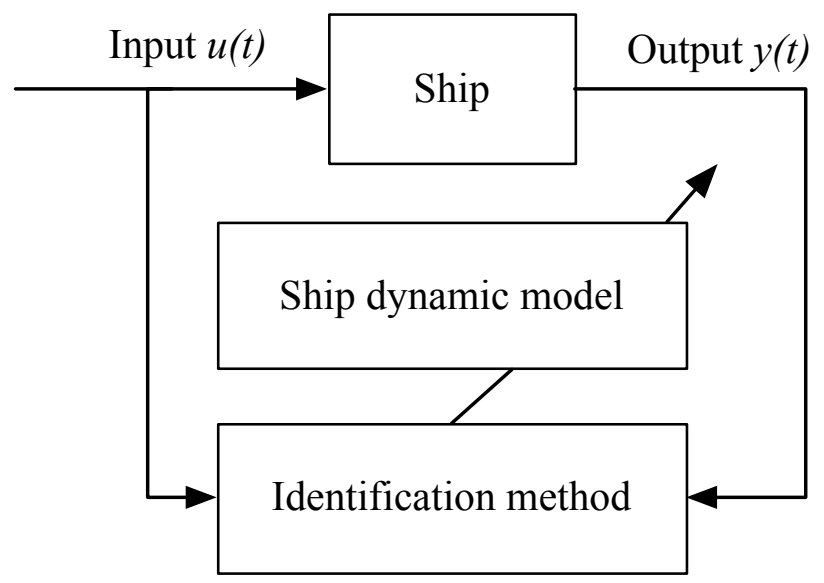

Figure 3.2.2: Basic framework of the identification of ship dynamic model.

Apart from the traditional methods, many intelligent algorithms have been developed for identifying ship dynamic models in recent years. The genetic algorithm (GA) and global search algorithm (GS) are utilized together by Erunsal (Erunsal 2015) to identify the model which was further applied to design controller. Ferri, et al. (Ferri, Manzi, Fornai, Ciuchi, and Laschi 2013) adopted the simulated annealing algorithm (SA) to identify the dynamic model of an autonomous surface vehicle (ASV) taking into account the performance of sensors used for measurements record. Bhattacharyya and Haddara (Bhattacharyya and Haddara 2006) employed the neural network method (NN) to estimate the hydrodynamic derivatives embedded in the nonlinear steering model. SVMs are firstly successively applied in 2009 by Luo and Zou (Luo and Zou 2009) in the maritime domain to estimate hydrodynamic derivatives of the Abkowitz model taking into account the free-running tests.

On the basis of those identification methods, some studies focus on simultaneously cooperating with two approaches and successively applying them to identify ship dynamic models. The examples are a recursive least square method combined with a recursive prediction error method for identifying ship maneuvering dynamics (Nguyen 2008), an EKF with constrained least square method used to estimate maneuvering parameters (Araki, Sadat-Hosseini, Sanada, Tanimoto, Umeda, and Stern 2012), a nonlinear prediction error method and a unscented Kalman filtering method for identifying dynamic models of a high-speed 
trimaran ferry (Herrero and Gonzalez 2012).

By analyzing the performance of these identification methods for estimating parameters in the ship dynamic model, it is easy to reveal some noticeable points. The traditional identification methods such as LS and ML present some inherent drawbacks, i.e., the identification results rely on either the mathematical model of the research object or the initial values of parameters needed to be predefined. The intelligent algorithm-based identification methods can overcome the drawbacks of traditional identification methods. But some methods involving GS, GA, SA, and $\mathrm{NN}$ cannot always guarantee the generalization performance and optimal solution and avoid the curse of dimensionality. Differently, SVMs based identification method can prevent these deficiencies and guarantee satisfying performance. That is due to the irreplaceable merits of SVMs. Firstly, SVMs can work well with finite samples. Then, it converts the complex optimization problem into a quadratic optimization problem which theoretically ensures the global optimum to be obtained. Thirdly, minimization of the structural risk promotes its generalization capability, and the curse of dimensionality can be omitted due to the computational complexity that is only related to the number of support vectors instead of the dimension of inputs. The performance of SVM based methods for either offline or online identification can benefit from these natural merits. Besides these attractive merits, one point of SVM based identification method are ought to be noticed, which is the stability problem associated with dealing with a large amount of data. The small-scale training set would result in local optimal solutions. But the large-scale training set could increase the computation burden due to a large size of kernel matrix. From the suggestion proposed by Suykens et al. (Suykens, Gestel, Brabanter, Moor, and Vandwalle 2002), the length of the training set is to be less than 2000. In addition, a robust method (i.e., optimal truncated SVM) with the ability of diminishing parameter uncertainty on demand of a low computational cost by reducing the dimensionality of the kernel matrix can be applied to solve this stability problem. In this study, attention to applying a small amount of training data less than 2000 to the offline identification of the simplified ship dynamic model is paid.

One noticeable deficiency of SVM is that its identification results are sensitive to the structural parameters in SVM such as the insensitivity factor, the regularization parameter, and kernel parameters, which means only if these parameters in SVM are defined by particular settings the globally optimal solutions can be guaranteed. Thus, the selection of these parameters for SVM is one of the most important steps when using it as an identifier. To remedy this deficiency of SVM, Frohlich et al. (Frohlich and Zell 2005) used the grid search on the log-scale of the parameter in combination with the cross-validation technique to tune parameters in SVM. Even though this 
technique is simple, it is often time-consuming and at average accuracy (Sulaiman, Mustafa, Shareef, and Khalid 2012). From the extensive literature reviews, many different approaches have been proposed for tuning parameters in SVM. One widely-used technique is the swarm intelligence, for instance, the PSO (Luo, Soares, and Zou 2016), the GA (Dioşan, Rogozan, and Pecuchet 2012), the firefly algorithm (Xiong, Bao, and Hu 2014), and ABC (Sulaiman et al. 2012). Apparently, the design of the developed hybrid model is different regarding the various problems needed to be solved in related domains. For the problem of parameter estimation of the ship dynamic model, the hybrid approach of ABC with SVM has not been applied, which will be studied and contributed to this thesis.

\subsubsection{Support Vector Machines}

Machine learning is always to find rules by learning from the observed data and then uses these derived rules to make predictions. Some machine learning algorithms are commonly dependent on the theoretical foundation, i.e., statistics. Traditional statistics are based on the asymptotic theory that the number of samples tends to infinity, whereas in practical problems, the number of samples usually tends to be limited. Statistical learning theory or VC theory started by Vapnik and Chervonenkis in the Sixties (Scholkopf and Smola 2002) provides a unified framework specialized to solve the problem of finite samples learning.

In 1999, SVMs as one type of machine learning algorithms based on statistical learning theory was primarily developed by Vapnik and co-workers since it was proposed (Schiilkop, Burgest, and Vapnik 1995) (Vapnik, Golowich, and Smola 1996). Built on the principles of structural risk minimization (SRM), SVMs can make the best compromise between the complexity of the learned model and the capacity of the learning algorithm for the given finite samples to ensure the best generalization performance. Different from some existing statistical algorithms involving the definition of probability measure and a massive number of rules, etc., SVMs illustrates three attractive points. One is that it can turn the learning task into a quadratic programming problem which generally can obtain the global optimal solution. The second one is that it can introduce the feature mapping function (which can also be induced by a kernel function) to cope with the issue about nonlinear inseparable inputs by mapping these input to a higher dimensional feature space where the best hyperplane is possibly found for these inputs. The third one is that it can make the computational complexity ultimately depend on the dimensionality of the input space and the complexity of the kernel function. More detailed information on SVMs can refer to the excellent book written by Vapnik (Vapnik 2013).

SVMs have been effectively applied to solve real-world problems, e.g., text and hypertext 
categorization, classification of images, hand-written characters recognition, predictions in the biological sciences (Burges 1998). The algorithm used for pattern recognition tasks is one extension of the SVM, so-called support vector classification algorithm (SVC). Another algorithm, i.e., SVR extended from SVM, is employed in regression cases.

Comprehensive tutorials on SVC in (Burges 1998) and on SVR in (Smola and Schölkopf 2004) are well reported. In this work, the SVR based identification method will be utilized for the identification of ship dynamic models.

\subsubsection{Linear Support Vector Classification Algorithm}

The Separable Case. Suppose the given training data $T=\left\{\left(\mathbf{X}_{1}, y_{1}\right), \cdots,\left(\mathbf{X}_{l}, y_{l}\right)\right\} \in(\chi \times y)^{l}$, in which $\mathbf{X}_{i} \in \chi=\mathbb{R}^{d}(i=1, \cdots, l)$ are patterns, and each pattern is a $p$-dimensional real vector, and $y_{i} \in y\{-1,1\}$ are labels, then to find a hyperplane to linearly separate all patterns for which the label $y_{i}=1$ belongs to, and for which $y_{i}=-1$ can be assigned. The SVC is to look for a hyperplane with maximum margin. For the classification of a set of points, the bigger the margin of the hyperplane is, the higher the confidence of the classification is.

A hyperplane for the SVC can be defined as a set of patterns $\mathbf{X}$ satisfy

$$
\left\{\mathbf{X} \in \mathbb{R}^{d}: f(\mathbf{X})=\mathbf{W} \cdot \mathbf{X}+b=o\right\}
$$

where $\mathbf{W}$ is normal to the hyperplane, $b$ is the intercept, $\mathbf{W} \cdot \mathbf{X}$ means the dot product between $\mathbf{W}$ and $\mathbf{X}, f(\mathbf{X})=\mathbf{W} \cdot \mathbf{X}+b$ denotes the decision function which calculates the distance of a pattern to the hyperplane. The hyperplane defined in $(3.11)$ is located in the middle of two other parallel hyperplanes $\left(H_{1}, H_{2}\right)$ expressed as $H_{1}: \mathbf{W} \cdot \mathbf{X}+b=1$ and $H_{2}: \mathbf{W} \cdot \mathbf{X}+b=-1$.

The distance between $H_{1}$ and $H_{2}$ is calculated by $\frac{2}{\|\mathbf{W}\|}$ in which $\|\mathbf{W}\|$ presents the Euclidean norm of $\mathbf{W}$. Therefore, the best maximum-margin hyperplane can be obtained by maximizing $\frac{2}{\|\mathbf{W}\|}$ concerning the constraints written in inequalities as follows

$$
\begin{gathered}
\mathbf{W} \cdot \mathbf{X}_{i}+b \geq 1 \text { for } y_{i}=1 \\
\mathbf{W} \cdot \mathbf{X}_{i}+b \leq-1 \text { for } y_{i}=-1
\end{gathered}
$$

from where one can see that the points located on the hyperplane $H_{1}$ also satisfy the equality relationship in (3.12), and the hyperplane $H_{2}$ matches the equality in $(3.13)$. These constraints prevent all training points from falling in the margin. For the sake of mathematical convenience, 
these constraints are defined by one combined inequality

$$
y_{i}\left(\mathbf{W} \cdot \mathbf{X}_{i}+b\right)-\mathbf{1} \geq 0, \forall i
$$

Afterward, the problem of finding the maximum-margin hyperplane is converted into the optimization problem concerning the maximization of $\frac{1}{\|\mathbf{W}\|}$ subject to constraints (3.14), for which the objective function is presented as

$$
\max \frac{1}{\|\mathbf{W}\|}, \quad \text { s.t. }, y_{i}\left(\mathbf{W} \cdot \mathbf{X}_{i}+b\right)-1 \geq 0, \forall i
$$

For the mathematical convenience, the objective function is modified into the following form as

$$
\min \frac{1}{2}\|\mathbf{W}\|^{2}, \quad \text { s.t. }, y_{i}\left(\mathbf{W} \cdot \mathbf{X}_{i}+b\right)-1 \geq 0, \forall i
$$

Now the optimization of the objective function can be regarded as solving a convex quadratic programming problem because in $(3.16)$ the objective function is a quadratic function and the constraints are linear constraints. The optimal solutions for convex quadratic programming problems are global optimums. Such a problem can be tackled by introducing Lagrange multipliers to form the Lagrange formulation of the problem presented as

$$
L(\mathbf{W}, b, a)=\frac{1}{2}\|\mathbf{W}\|^{2}-\sum_{i=1}^{l} a_{i}\left(y_{i}\left(\mathbf{W} \cdot \mathbf{X}_{i}+b\right)-1\right)
$$

Let minimize $L(\mathbf{W}, b, a)$ with respect to $\mathbf{W}, b$, and simultaneously require that the derivatives of $L(\mathbf{W}, b, a)$ with respect to all $a_{i}$ vanish, all subject to the constraints $a_{i} \geq 0, \forall i$. The similar results from above procedure can also occur in the process of solving a dual problem. That means maximization of $L(\mathbf{W}, b, a)$ subject to the constraints about the vanishment of the gradient of $L(\mathbf{W}, b, a)$ with respect to $\mathbf{W}, b$ vanish, and subject to the constraints about $a_{i} \geq 0, \forall i$ (Burges $1998)$. W, $b$ and $a$ can be calculated by applying the steps for solving a dual problem:

(1) Partially derive $\frac{\partial L(\mathbf{w}, b, a)}{\partial \mathbf{w}}=\mathrm{o} \Longrightarrow \mathbf{W}=\sum_{i=1}^{l} a_{i} y_{i} \mathbf{X}_{i}$ and $\frac{\partial L(\mathbf{w}, b, a)}{\partial b}=0 \Longrightarrow \sum_{i=1}^{l} a_{i} y_{i}=0$, then substitute the above results into the expression of $L(\mathbf{W}, b, a)$ to get the form of 
$L(\mathbf{W}, b, a)$ only with $a$

$$
L(\mathbf{W}, b, a)=\sum_{i=1}^{l} \alpha_{i}-\frac{1}{2} \sum_{i, j=1}^{l} \alpha_{i} \alpha_{j} y_{i} y_{j}\left(\mathbf{X}_{i} \cdot \mathbf{X}_{j}\right)
$$

(2) Maximize $\sum_{i=1}^{l} a_{i}-\frac{1}{2} \sum_{i, j=1}^{l} a_{i} \alpha_{j} y_{i} y_{j}\left(\mathbf{X}_{i} \cdot \mathbf{X}_{j}\right)$ with respect to $\alpha_{i}$ subject to $\alpha_{i} \geq 0, \forall i$ and $\sum_{i=1}^{l} a_{i} y_{i}=0$, then apply Sequential Minimal Optimization (SMO) algorithm to calculate the $a$. Details concerning SMO algorithm refer to (Platt 1999).

(3) Calculate the intercept by $b=-\frac{\max _{i: y_{i}=-1} \mathbf{W} \cdot \mathbf{X}_{i}+\min _{i: y_{i}=1} \mathbf{W} \cdot \mathbf{X}_{i}}{2}$. Afterward, the decision function and the maximum-margin hyperplane are obtained by substituting $\mathbf{W}, b$ into $f(\mathbf{X})=\mathbf{W} \cdot \mathbf{X}+b$ and (3.11). The decision function becomes

$$
f(\mathbf{X})=\left(\sum_{i=1}^{l} a_{i} y_{i} \mathbf{X}_{i}\right)^{T} \mathbf{X}+b=\sum_{i=1}^{l} a_{i} y_{i}<\mathbf{X}_{\mathbf{i}} \cdot \mathbf{X}>+b
$$

It is worthy to note that the data on the hyperplanes $H_{1}$ and $H_{2}$ are called support vectors, for which the Lagrange multipliers are greater than zero $\left(a_{i} \geq 0, \forall i\right)$. For the data outside the $H_{1}$ and $H_{2}$, their Lagrange multipliers are set as zero $\left(a_{i}=0, \forall i\right)$.

The Non-Separable Case. The above algorithm is applied to separate the data into two classes, but it is not suitable to handle the case in which there are non-separable data because of the outliers. To deal with the non-separable case, the non-negative slack variables $\xi_{i}(i=1, \cdots, l)$ are added into the constraints $(3.14)$

$$
y_{i}\left(\mathbf{W} \cdot \mathbf{X}_{i}+b\right)-1+\xi_{i} \geq 0, \forall i: \xi_{i} \geq 0
$$

The slack variables can be calculated by the hinge loss function

$$
\xi_{i}=\max \left(\mathrm{o}, 1-y_{i}\left(\mathbf{W} \cdot \mathbf{X}_{i}+b\right)\right), \forall i
$$

from which, one can get an understanding of how every pattern locates in reference to the hyperplane. If the function is zero, the related pattern lies on the correct side of the hyperplane. If the function is bigger than one, the pattern lies on the incorrect side of the hyperplane.

Otherwise, the pattern lies in the margin.

With the introduction of an extra error term $C \sum_{i=1}^{l} \xi_{i}$, the objective function is then rewritten 
as

$$
\min \frac{1}{2}\|\mathbf{W}\|^{2}+C \sum_{i=1}^{l} \xi_{i}, \quad \text { s.t. }, y_{i}\left(\mathbf{W} \cdot \mathbf{X}_{i}+b\right)-1+\xi_{i} \geq 0, \forall i: \xi_{i} \geq 0
$$

where $C$ is a user-defined parameter used to decide the penalty for the errors. The bigger the setting of $C$ is, the higher the penalty for errors is, in other words, the margin is smaller. The objective function $(3.21)$ can also be transferred into the Lagrange formulation, which is presented as

$$
L(\mathbf{W}, b, a, \mu)=\frac{1}{2}\|\mathbf{W}\|^{2}+C \sum_{i=1}^{l} \xi_{i}-\sum_{i=1}^{l} \alpha_{i}\left(y_{i}\left(\mathbf{W} \cdot \mathbf{X}_{i}+b\right)-1+\xi_{i}\right)-\sum_{i=1}^{l} \mu_{i} \xi_{i}
$$

in which $\mu_{i}, \forall i$ are Lagrange multipliers with the lower bound $\mu_{i} \geq 0, \forall i$.

Applying the steps in the process of solving Lagrange formulation to the problem of $(3.22)$ leads to the following results.

(1) The results from partial derivatives are

$$
\left\{\begin{array}{llc}
\frac{\partial L(\mathbf{w}, b, \alpha, \mu)}{\partial \mathbf{w}} & =\mathrm{o} \Longrightarrow & \mathbf{W}=\sum_{i=1}^{l} a_{i} y_{i} \mathbf{X}_{i} \\
\frac{\partial L(\mathbf{w}, b, \alpha, \mu)}{\partial b} & =0 \Longrightarrow & \sum_{i=1}^{l} a_{i} y_{i}=\mathrm{o} \\
\frac{\partial L(\mathbf{w}, b, \alpha, \mu)}{\partial \xi_{i}} & =0 \Longrightarrow & C-a_{i}-\mu_{i}=0, \forall i
\end{array}\right.
$$

(2) The result got by substituting the results of (1) to (3.22) is

$$
L(\mathbf{W}, b, a, \mu)=\sum_{i=1}^{l} a_{i}-\frac{1}{2} \sum_{i, j=1}^{l} a_{i} \alpha_{j} y_{i} y_{j}\left(\mathbf{X}_{i} \cdot \mathbf{X}_{j}\right) \text { which is a dual formulation. }
$$

Subsequently, the solution of a dual formulation is applied to calculate the $a_{i}, \forall i$ and then the $\mu_{i}, \forall i$.

From the above results, one can see that an upper bound is added to $a_{i}\left(a_{i} \leq C\right)$, which is derived from $C-a_{i}-\mu_{i}=0, \forall i$ and $\mu_{i} \geq 0$. Additionally, the $\mathbf{W}$ obtained in the non-separable case is nearly as same as the one in the separable case. The left task is to calculate $b$, which can be solved by following the suggestions from Burges (Burges 1998) by applying patterns satisfying $\mathrm{o}<a_{i}<C$ to equation $a_{i}\left(y_{i}\left(\mathbf{W} \cdot \mathbf{X}_{i}+b\right)-1+\xi_{i}\right)=\mathrm{o}$ with $\xi_{i}=\mathrm{o}$. This solution reveals that the patterns used to compute $b$ are support vectors that lie on the boundaries of the margin. 


\subsubsection{Nonlinear Support Vector Classification Algorithm}

Generally, the linear decision function of the patterns is unable to find an optimal hyperplane for some cases where the input space is nonlinear. To handle this problem, one solution is to introduce a mapping function called $\Phi$ to map the patterns in input space to a high-dimensional feature space $\mathfrak{H}$ where a linear decision function can be found. Such a linear decision function constructed in the feature space is given by

$$
f(\mathbf{X})=\mathbf{W} \cdot \Phi\left(\mathbf{X}_{i}\right)+b
$$

Afterward, the objective function subject to the constraints with the transformation of patterns from the input space to the feature space yields

$$
\min \frac{1}{2}\|\mathbf{W}\|^{2}, \quad \text { s.t. } y_{i}\left(\mathbf{W} \cdot \Phi\left(\mathbf{X}_{i}\right)+b\right)-1 \geq 0, \forall i
$$

This objective function is still a quadratic programming formulation. Now, it seems the mapped patterns in feature space are linearly separable. But it is still challenging to explicitly deal with $\Phi$. So far, the benefit of kernel trick $K\left(\mathbf{X}_{i}, \mathbf{X}\right)$ is applied, which is effective to calculate the dot product of patterns in the feature space. In (Hofmann, Schölkopf, and Smola 2008)), more details about kernel trick can be found. Therefore, the solution used previously for a quadratic programming problem can be applied for $(3.24)$ to get the decision function.

(1) Firstly, obtain $\mathbf{W}=\sum_{i=1}^{l} a_{i} y_{i} \Phi\left(\mathbf{X}_{i}\right)$.

(2) Calculate $\alpha_{i}$ by maximizing $\sum_{i=1}^{l} \alpha_{i}-\frac{1}{2} \sum_{i, j=1}^{l} \alpha_{i} \alpha_{j} y_{i} y_{j}\left(\Phi\left(\mathbf{X}_{i}\right) \cdot \Phi(\mathbf{X})\right)$, subject to $\sum_{i=1}^{l} a_{i} y_{i}=\mathrm{o}$ and $\alpha_{i} \geq \mathrm{o}$.

(3) Apply support vectors in the feature space to compute $b$ by

$$
b=\mathbf{W} \cdot \Phi(\mathbf{X})-y_{i}=\sum_{i=1}^{l} a_{i} y_{i} K\left(\mathbf{X}_{i}, \mathbf{X}\right)-y_{i} .
$$

(4) Finally, the decision function used to classify new pattern is given as

$$
f(\mathbf{X})=\left(\sum_{i=1}^{l} a_{i} y_{i} \Phi\left(\mathbf{X}_{i}\right)\right)^{T} \Phi(\mathbf{X})+b=\sum_{i=1}^{l} \alpha_{i} y_{i} K\left(\mathbf{X}_{i}, \mathbf{X}\right)+b
$$

It is deserved to emphasize that only the kernel function meeting Mercer's condition is applicable for the quadratic programming problem. That means $K\left(\mathbf{X}_{i}, \mathbf{X}\right)=\sum_{p=0}^{\infty} c_{p}\left(\mathbf{X}_{i}, \mathbf{X}\right)^{p}$ with 
$c_{p}>\mathrm{o}$ is uniformly convergent. The popularly used kernel functions satisfying Mercer's condition are illustrated:

(1) Polynomial: $K\left(\mathbf{X}_{i}, \mathbf{X}\right)=\left(\left(\mathbf{X}_{i} \cdot \mathbf{X}\right)+d\right)^{p}$

(2) Gaussian radial basis function: $K\left(\mathbf{X}_{i}, \mathbf{X}\right)=\exp \left(-\gamma\left\|X_{i}-X\right\|^{2}\right)$ with $\gamma>$ o or $\gamma=\frac{1}{2 \sigma^{2}}$

(3) Linear function: $K\left(\mathbf{X}_{i}, \mathbf{X}\right)=\left(\mathbf{X}_{i} \cdot \mathbf{X}\right)$

(4) Sigmoid function: $K\left(\mathbf{X}_{i}, \mathbf{X}\right)=\tanh \left(c\left(\mathbf{X}_{i} \cdot \mathbf{X}\right)+d\right), c>0, d>0$

(5) Fourier function: $K\left(\mathbf{X}_{i}, \mathbf{X}\right)=\frac{\sin (N+0.5)\left(\mathbf{X}_{i}-\mathbf{X}\right)}{\sin \left(0.5\left(\mathbf{X}_{i}-\mathbf{X}\right)\right)}$

\subsubsection{Support Vector Regression Algorithm}

SVR is expended from SVC by introducing the $\mathcal{\varepsilon}$-insensitive loss function which guarantees the sparsity of SVR. Here one can start from a case where a function that can fit the patterns in the high dimensional input space is needed to be found. Analogous to the nonlinear SVC, the feature mapping function is applied, which yields the decision function as

$$
f(\mathbf{X})=\mathbf{W} \cdot \Phi\left(\mathbf{X}_{i}\right)+b \text { with } \mathbf{W} \in \chi, b \in \mathbb{R}
$$

Then the $\mathcal{\varepsilon}$-insensitive loss function is given

$$
|y-f(\mathbf{X})|_{\varepsilon}=\left\{\begin{array}{cc}
0, & \text { if }:|y-f(\mathbf{X})| \leq \varepsilon \\
|y-f(\mathbf{X})|-v, & \text { otherwise }
\end{array}\right.
$$

which means that only the patterns (training data) outside and on the boundary of the $\mathcal{E}$-insensitive loss zone contribute to the determination of the decision function. These patterns are called support vectors. One important aspect of SVR is to ensure its flatness while finding the decision function, which can be done through minimizing $\|\mathbf{W}\|^{2}$. To deal with the non-separable case, the non-negative slack variables $\xi_{i}, \xi_{i}^{*}(i=1, \cdots, l)$ are applied. The optimization formulation is established under the consideration of extra errors, which is given as

$$
\min \frac{1}{2}\|\mathbf{W}\|^{2}+C \sum_{i=1}^{l}\left(\xi_{i}+\xi_{i}^{*}\right) \quad \text { s.t., }\left\{\begin{array}{cc}
y_{i}-\left(\mathbf{W} \cdot \mathbf{X}_{i}-b\right) & \leq \varepsilon+\xi_{i} \\
\left(\mathbf{W} \cdot \mathbf{X}_{i}\right)+b-y_{i} & \leq \varepsilon+\xi_{i}^{*} \\
\xi_{i}, \xi_{i}^{*} & \geq 0, \forall i
\end{array}\right.
$$


where $C$ is the regularization parameter and always positive, which adjusts the equilibrium between the flatness and the tolerance to deviations greater than $\mathcal{\varepsilon}$. The optimization problem is transformed into the dual problem and the related solution is given by

$$
\begin{gathered}
\mathbf{W}=\sum_{i=1}^{l}\left(a_{i}-a_{i}^{*}\right) \Phi\left(\mathbf{X}_{i}\right) \\
f(\mathbf{X})=\left(\sum_{i=1}^{l}\left(a_{i}-a_{i}^{*}\right) \Phi\left(\mathbf{X}_{i}\right)\right)^{T} \Phi(\mathbf{X})+b=\sum_{i=1}^{l}\left(a_{i}-a_{i}^{*}\right) K\left(\mathbf{X}_{i}, \mathbf{X}\right)+b
\end{gathered}
$$

Lastly, Figure 3.2.3 graphically illustrates framework of SVR (Smola and Schölkopf 2004).

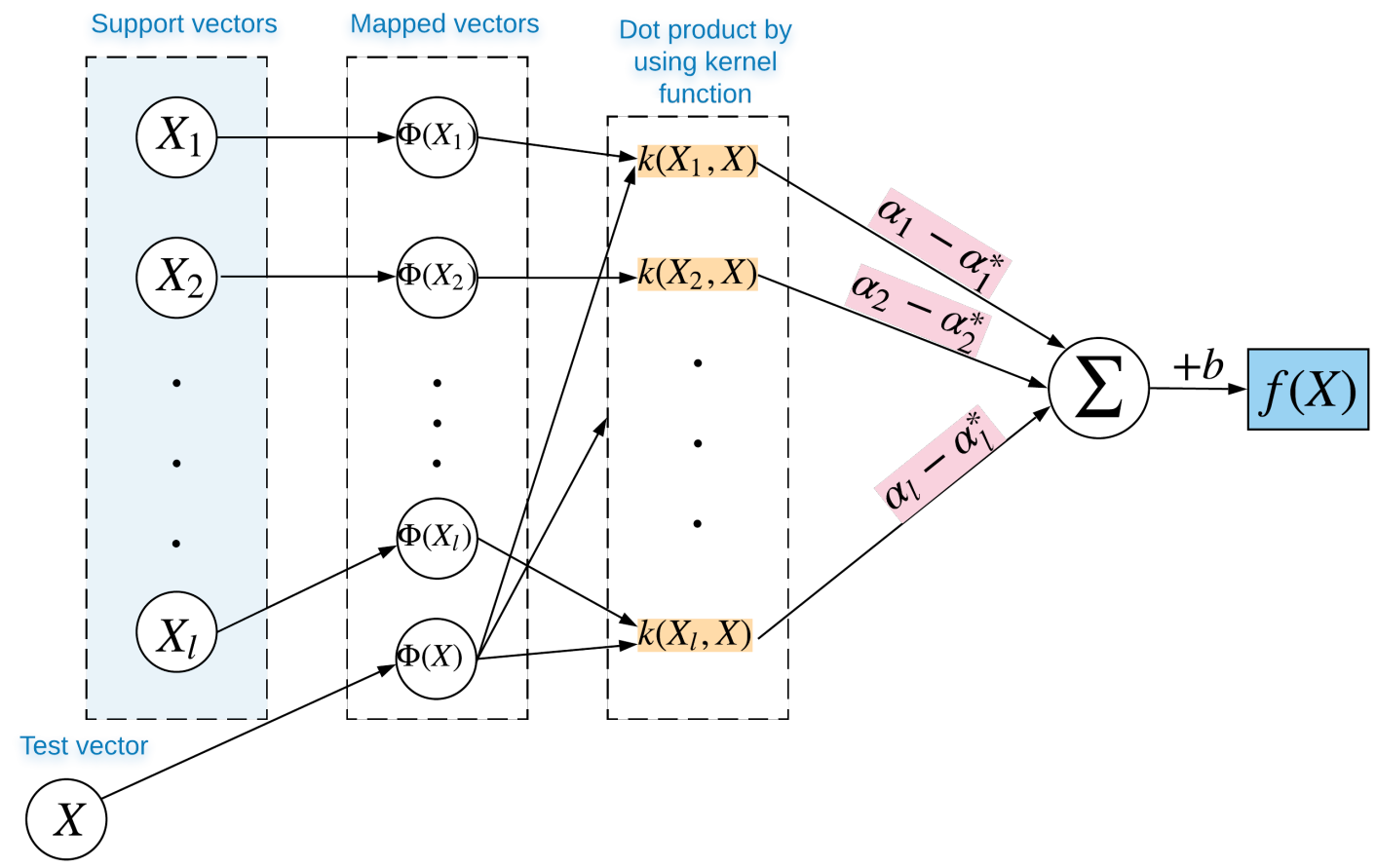

Figure 3.2.3: Graphical illustration of SVR.

\subsubsection{Least Seuare Support Vector Regression Algorithm}

As one modified version of SVR, the least square support vector regression algorithm (LS-SVR) proposed by Suykens et al. (Suykens and Vandewalle 1999) works with equality constraints instead of inequality constraints and the quadratic deviation. Even though such changes in 
LS-SVR, it still inherits the merits of SVR and can be used for finite samples to find a function of nonlinear system estimation. Suppose the decision function is

$$
f(\mathbf{X})=\mathbf{W} \cdot \Phi\left(\mathbf{X}_{i}\right)+b \text { with } \mathbf{W} \in \chi, b \in \mathbb{R}
$$

Then the optimization problem with equality constraints becomes like

$$
\min \frac{1}{2}\|\mathbf{W}\|^{2}+\frac{C}{2} \sum_{i=1}^{l} e_{i}^{2}, \quad \text { s.t. }, y_{i}=\mathbf{W} \cdot \Phi\left(\mathbf{X}_{i}\right)+b+e_{i}
$$

where $e_{i}(i=1, \cdots, l)$ are regression errors. As previous process, the Lagrange formulation of the optimization problem is given as

$$
L(\mathbf{W}, b, a, e)=\frac{1}{2}\|\mathbf{W}\|^{2}+\frac{C}{2} \sum_{i=1}^{l} e_{i}^{2}-\sum_{i=1}^{l} a_{i}\left(\mathbf{W} \cdot \Phi\left(\mathbf{X}_{i}\right)+b+e_{i}-y_{i}\right)
$$

Now the derivatives with respect to $\mathbf{W}, b, e_{i}$, and $\alpha_{i}$ are computed and set to be zero, respectively.

$$
\left\{\begin{array}{llc}
\frac{\partial L(\mathbf{w}, b, a, e)}{\partial w}=0 \Longrightarrow & \mathbf{W}=\sum_{i=1}^{l} a_{i} \Phi\left(\mathbf{X}_{i}\right) \\
\frac{\partial L(\mathbf{w}, b, a, e)}{\partial b}=0 \Longrightarrow & \sum_{i=1}^{l} a_{i}=0 \\
\frac{\partial L(\mathbf{w}, b, a, e)}{\partial e_{i}}=0 \Longrightarrow & a_{i}=C e_{i} \\
\frac{\partial L(\mathbf{w}, b, a, e)}{\partial a_{i}} & =0 \Longrightarrow & \mathbf{W} \cdot \Phi\left(\mathbf{X}_{i}\right)+b+e_{i}-y_{i}=0
\end{array}\right.
$$

After straightforward computations, variables $\mathbf{W}$ and $e_{i}$ are eliminated from (3.34) at the same time the kernel trick is applied. Then the decision function yields

$$
f(\mathbf{X})=\sum_{i=1}^{l} a_{i} K\left(\mathbf{X}_{i}, \mathbf{X}\right)+b
$$

\subsubsection{Requirement Fulfillment Summary}

The execution of identification of ship dynamic models is trickily dependent on the procedure of system identification technique including core four steps, i.e., acquisition of observed data, determination of the set of model structure, the decision of identification method, and validation of the identified model. In maritime engineering, the maneuver to supply informative data suggests zigzag maneuver which can provide consistent excitation signals of ship motions. The 
ship dynamic model studied in this thesis is classified as the grey-box model. The final step in validating the identified model can be directly carried out by judging the degree of agreement between the compatible outputs of an identified model and original maneuvers. Unlike these three steps, the selection of the identification method is conducted effectively according to the comprehensive survey on the applications of various identification methods in the maritime domain, which is illustrated as follows.

- Among conventional methods, examples are LS, ML, KF, and their-related modified versions, which are not intelligent methods. LS with simple expressions is easy-understanding and widely-used in engineering but it is sensitive to the outliers corrupted in observed data caused by environmental disturbances and sensor noises due to its squared norm minimization, which implies that this method can partially fulfill the seventh requirement. $\mathrm{ML}$ and KF can be regarded as recursive methods on demand of parameter values predetermined based on empiric, which indicates that these methods can partially fulfill the fifth and seventh requirements.

- Among intelligent methods, instances are GA, GS, AS, NN, and SVM based identification methods. GA, GS, and AS based identification methods can correctly identify ship dynamic models if the observed data are preprocessed, but they have no ability to often ensure the global optimum. Besides the local optimum, $\mathrm{NN}$ also has a problem of dimensionality curse. So, these methods fulfill the sixth requirement and partially fulfill the seventh requirement. SVM based identification method avoids the above shortcomings, from which one can conclude that this method fulfills the fifth and sixth requirements and partially fulfills the seventh requirement.

- Among hybrid methods, cases are recursive least square method combined with recursive prediction error method, EKF with constrained least square method, nonlinear prediction error method incorporated with unscented KF method. The combination of two identification methods can improve the robustness and identification performance on obtaining global optimal solutions but it is not intelligent. So, one can learn that these hybrid methods fulfill the fifth and seventh requirements.

One remark can be concluded that the derived requirements on the identification method are not fully fulfilled. 


\subsection{Objective Coverage Summary}

The overview of the state-of-the-art ship dynamic model and system identification is the basis for arising a solution to the research question. To which degree the previously posed objectives are covered is summarized.

Objective 1 (Selection of an existing $6 \mathrm{DOF}$ model as the fundamental model for model simplification and modification.) is covered. Three typical dynamic models including the Abkowitz model, MMG model, and vectorial representation are potential selections used to describe ship motions in 6 DOF. By comprehensively analyzing these models, in particular, the two folds, i.e., advantages and disadvantages, the ship dynamic model expressed in the matrix-vector form shows more attractive advantages which are illustrated as follows: The vectorial representation can completely describe 6 DOF dynamics of the ship. Each term of the representation has a physical interpretation. Some properties of the ship can be trivial to be exploited through passivity or stability analysis. The model presented in the vector-matrix form is easy-to-use for model simulation, which is very useful to simulate ship maneuvering performance once obtaining the dynamic model. Thus, the dynamic model expressed in matrix-vector form can serve as the fundamental model to provide a simplified model through reasonable model simplification and modification.

Objective 2 (Simplification of the model dependent on trade-offs between accuracy and complexity.) is partially covered. Even though the 6 DOF dynamic model, i.e., the vectorial representation, can be simplified to 3 DOF model with acceptable precision based on a series of reasonable assumptions, such simplification is specific to a research object but not effective for more than one research objects at the same time. The trade-offs between accuracy and complexity should be adopted for the simplification of a dynamic model for different types of ships rather than for only one ship.

Objective 3 (Comparison and selection of identification methods.) is partially covered. The study on applying two collaborative identification methods, e.g., the aforementioned combination of recursive least square method and recursive prediction error method, and the cooperation of EKF and LS, to identify ship dynamic model presented the comparison between the collaborative method and single method. However, these studies are not sufficient for this study to select a suitable identification method due to the inherent deficiencies (mentioned previously in summary on system identification) of the method studied. Therefore, the selection of a powerful identification method has to be done in this thesis, which can be on the basis of comparing the performance of the intelligent method and the widely-used method on identifying 
a ship dynamic model with a simple structure.

Objective 4 (Improvement of the identification method.) is partially covered. The LS was improved to extend LS to mitigate the negative influence caused by the noise corrupted in the measurements. Additionally, the initial KF was modified to EKF or other modified versions so that it is applicable for both linear systems and nonlinear systems. More similar cases can be found in the related work, but the inherent drawback of the initial method cannot be avoided to past to the modified version. Much differently, the SVM based identification method shows few poornesses except the suitable settings of its structural parameters to control the trade-off between empirical risk and confidence interval. To satisfy this requirement, methods such as cross-validation method and particle swarm optimization algorithm (PSO) have been used to optimize the particular settings of parameters in SVM. However, the performance of SVM was not entirely improved by using either the cross-validation method or PSO. This is due to that these methods cannot always guarantee the particular settings of parameters in SVM. Hence, a stronger global optimization algorithm is expected.

Objective 5 (Parameter estimation tests by using simulated and experimental data.) is not covered. From the introduced studies on identifying ship dynamic models by applying system identification technique, there is no kind of tests simultaneously adopting simulated data and experimental data stemmed from ship maneuvers. The simulated data are always used to verify the identification performance of the parameter estimation method by comparing the estimated values and predetermined values of parameters. The experimental data extracted from particular trails of real ships, e.g., Esso Osaka tanker, Arleigh Burke Class Destroyer and Charlie 2005, are utilized to identify and acquire the dynamic model specific to a real ship, in which the dynamic model is derived based on physical laws depending on the application purpose, and some differences between models can be observed due to the specific characteristics of different ships. 


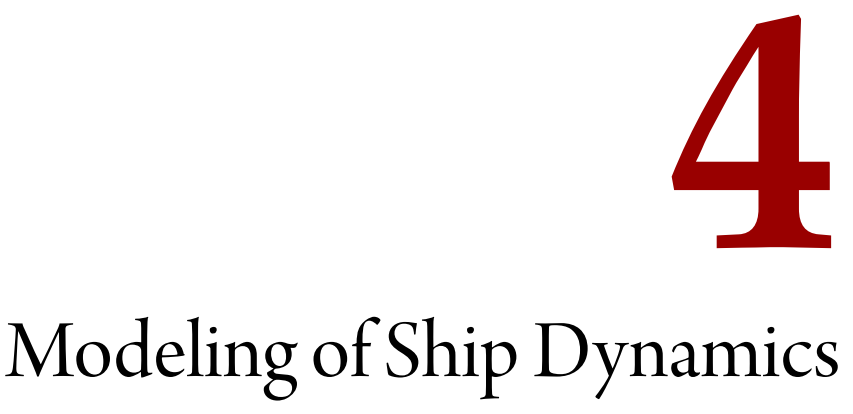

As elaborated in related work on ship dynamic model, each typical model, e.g., Abkowitz model, MMG model, or vectorial representation model, can completely describe ship dynamics, but a number of parameters involved in the model make challenges to its application. This is due to the tough task of estimating the unknown parameters. To estimate all these parameters, optimal experiments are required to be designed to acquire informative observations, which are of many difficulties including but not limited to the weather condition, the equipment, the financial support. Additionally, the problem of parameter identifiability is hard to be avoided due to multicollinearity happens much frequently for the complicated model with a number of parameters. Furthermore, the ship dynamic model studied is specific to a ship. Switching the application of the model between different types of the ship is on demand of re-setup experiments, which is rarely found.

The vectorial representation model has a piece of irreplaceable advantage which is that the nonlinear system properties such as symmetry, skew-symmetry, and positiveness of matrices can be exploited through the passivity and stability analysis. These properties are helpful for the model user to analyze the characteristics of the ship. In addition, the model expressed in vector-matrix form for simulation is easy to be conducted in most programming languages which 
means a lot to the application purpose of the obtained dynamic model. Therefore, in this chapter, the vectorial representation model is selected as the fundamental form for simplification and modification to derive a required model. The reduced-order version expressed in $3 \mathrm{DOF}$ based on the previously introduced 6 DOF version is explained in Section 4.1, and further employed to obtain the simplified ship dynamic model containing the decoupled surge and steering models as presented in Section 4.3 where the Nomoto models are also derived based on the reduced-order steering model. The forces and moments acting on ships are calculated by the function of propeller thrust and rudder angle in Section 4.2.

The basic structure of the model established based on Newton's second laws of motion is applicable for ships that are regarded as rigid-body objects. The determination of forces and moments by using the function of rudder deflection and propeller shaft speed makes it easy to undertake local changes on the model according to varying properties of different ships. The requirements, e.g., $\mathrm{R}_{1-4}$, are reflected in the process of modeling ship dynamics in this chapter.

\subsection{DOF Ship Dynamic Model}

In order to release the difficulty of the parameter identification, the complexity of the 6 DOF ship dynamic model decreased through the simplification based on a set of reasonable assumptions as follows: (1) surface ships are moving in a horizontal plane in the ideal fluid; (2) ships masses are uniformly distributed; (3) the body-fixed frame coincides with the center of gravity; $(4)$ both the center of gravity and the center of buoyancy point vertically along the z-axis; (5) ships are the port-starboard symmetry and (6) the horizontal dynamic motions are decomposed into speed (surge) and steering (sway and yaw) motion ( $\mathrm{Liu}, \mathrm{Zhang}$, Yu, and Yuan 2016). Therefore, the two mainly concerned models, i.e., steering and speed models, are extracted to describe 3 DOF horizontal motions. The former model can be used to control the yaw angle by using the rudder angle as the control variable. The latter model is applicable to control the ship speed by varying the propeller shaft speed. To explain the simplification, the 3 DOF dynamic model (4.2) and the corresponding kinematic equations (4.1) governed based on the above assumptions are introduced as follows (Fossen 1994). The resultant forward speed is calculated by $U=\sqrt{u^{2}+v^{2}}$.

$$
\begin{gathered}
{\left[\begin{array}{c}
\dot{x} \\
\dot{y} \\
\dot{\psi}
\end{array}\right]=\left[\begin{array}{ccc}
\cos \psi & -\sin \psi & 0 \\
\sin \psi & \cos \psi & 0 \\
0 & 0 & 1
\end{array}\right]} \\
M \dot{v}+C(v) v+D(v) v+g(\eta)=\tau
\end{gathered}
$$


where the expression of each symbol is shown as

$$
\begin{aligned}
& v=[u, v, r]^{T} \\
& g(\eta)=[\mathrm{o}, \mathrm{o}, \mathrm{o}]^{T} \\
& \tau=\left[\tau_{X}, \tau_{Y}, \tau_{N}\right]^{T} \\
& M=M_{R B}+M_{A} \\
& =\left[\begin{array}{ccc}
m & 0 & 0 \\
0 & m & m x_{g} \\
0 & m x_{g} & I_{z}
\end{array}\right]+\left[\begin{array}{ccc}
-X_{\dot{u}} & 0 & 0 \\
0 & -Y_{\dot{v}} & -Y_{\dot{r}} \\
0 & -N_{\dot{v}} & -N_{\dot{r}}
\end{array}\right] \\
& =\left[\begin{array}{ccc}
m-X_{\dot{u}} & 0 & 0 \\
0 & m-Y_{\dot{v}} & m x_{g}-Y_{\dot{r}} \\
0 & m x_{g}-N_{\dot{v}} & I_{z}-N_{\dot{r}}
\end{array}\right] \\
& C(v)=C_{R B}(v)+C_{A}(v) \\
& =\left[\begin{array}{ccc}
0 & 0 & -m\left(x_{g} r+v\right) \\
0 & 0 & m u_{\circ} \\
m\left(x_{g} r+v\right) & -m u_{\circ} & 0
\end{array}\right] \\
& +\left[\begin{array}{ccc}
0 & \circ & Y_{\dot{v}} v+Y_{\dot{r}} r \\
0 & 0 & -X_{\dot{u}} u_{\circ} \\
-Y_{\dot{v}} v-N_{\dot{v}} r & X_{\dot{u}} u_{\circ} & 0
\end{array}\right] \\
& =\left[\begin{array}{ccc}
0 & 0 & Y_{\dot{v}} v+Y_{\dot{r}} r-m\left(x_{g} r+v\right) \\
0 & 0 & \left(m-X_{\dot{u}}\right) u_{\mathrm{o}} \\
m\left(x_{g} r+v\right)-Y_{\dot{v}} v-N_{\dot{v}} r & -\left(m-X_{\dot{u}}\right) u_{\mathrm{o}} & \circ
\end{array}\right]
\end{aligned}
$$




$$
\begin{aligned}
D(v)= & D_{L}+D_{N L}(v) \\
= & {\left[\begin{array}{ccc}
-X_{u} & 0 & 0 \\
0 & -Y_{v} & -Y \\
0 & -N_{v} & -N_{r}
\end{array}\right] } \\
+ & {\left[\begin{array}{ccc}
-X_{|u| u}|u| & 0 & 0 \\
0 & -Y_{|v| v}|v|-Y_{|r| v}|r| & -Y_{|r| r}|r|-Y_{|v| r|v|} \\
0 & -N_{|v| v}|v|-N_{|r| v}|r| & -N_{|r| r}|r|-N_{|v| r} v
\end{array}\right] }
\end{aligned}
$$

Eventually, the expressions of the 3 DOF model for describing surge, sway, and yaw motions are given as

$$
\left\{\begin{array}{c}
\tau_{X}=\left(m-X_{\dot{u}}\right) \dot{u}+\left(Y_{\dot{v}}-m\right) v r+\left(Y_{\dot{r}}-m x_{g}\right) r^{2}-X_{u} u-X_{|u| u}|u| u \\
\tau_{Y}=\left(m-Y_{\dot{v}}\right) \dot{v}+\left(m x_{g}-Y_{\dot{r}}\right) \dot{r}+\left(m-X_{\dot{u}}\right) u_{\mathrm{o}} r-Y_{v} v-Y_{|v| v}|v| v \\
-Y_{|r| v}|r| v-Y_{r} r-Y_{|v| r}|v| r-Y_{|r| r}|r| r \\
\tau_{N}=\left(m x_{g}-N_{\dot{v}}\right) \dot{v}+\left(I_{z}-N_{\dot{r}}\right) \dot{r}-\left(Y_{\dot{v}}-m\right) u v-\left(N_{\dot{v}}-m x_{g}\right) u r \\
-\left(m-X_{\dot{u}}\right) u_{\mathrm{o}} v-N_{v} v-N_{|v| v}|v| v-N_{|r| v}|r| v-N_{r} r \\
-N_{|v| r}|v| r-N_{|r| r}|r| r
\end{array}\right.
$$

which will be used as the fundamental 3 DOF model for the following simplification to obtain initial version of simplified dynamic model for this thesis.

\subsection{ACtuator Forces ANd Moments}

The actuator forces and moments are generated by a set of thrusters with revolutions per second $n=\left[n_{1}, n_{2}, \cdots, n_{p_{1}}\right]^{T}$ and a set of control surfaces (or propeller blade pitch) with angles $\delta=\left[\delta_{1}, \delta_{2}, \cdots, \delta_{p_{2}}\right]^{T}$. They are related to the vector $\tau$ through the mapping

$$
\tau=\left[\tau_{X}, \tau_{Y}, \tau_{N}\right]^{T}=B f\left(v_{r}, n, \delta\right)
$$

where $B$ is an actuator configuration matrix, $f\left(v_{r}, n, \delta\right)$ is a function that for each velocity $v_{r}$ relates the actuator set-point $(n, \delta)$ to a vector of forces. The propeller provides the thrust forces $\{T\}$ while the rudders generate lift forces $\{L\}$ and drag forces $\{D\}$. Here through disregarding the drag forces, the force vector can be described as

$$
f\left(v_{r}, n, \delta\right)=[T, L]^{T}
$$


Let the force attack points of $T$ located at coordinate $\left(x_{n}, y_{n}, z_{n}\right)$ in the body-fixed frame, and likewise $\left(x_{\delta}, y_{\delta}, z_{\delta}\right)$ for the rudders. Then the actuator configuration matrix is

$$
B=\left[\begin{array}{cc}
1 & 0 \\
0 & 1 \\
-y_{n} & x_{\delta}
\end{array}\right]
$$

According to references (Fossen 1994) and (Lindegaard and Fossen 2003), the thrust force can be expressed as

$$
T=T_{|n| n}|n| n, \quad T_{|n| n}>0
$$

where $n$ represents the propeller revolutions, $T_{|n| n}$ is a parameter decided depending on the propeller's diameter $D$ and the water density $\rho$.

From foil theory (Newman 2018), the lift forces are modeled as

$$
L=\frac{\rho}{2} A_{R} C_{L}(\delta)\left|u_{R}\right| u_{R}
$$

with

$$
C_{L}(\delta)=c_{1} \delta-c_{2}|\delta| \delta
$$

where $A_{R}$ is the effective rudder area, $u_{R}$ is the relative velocity of the fluid at the rudder surface, $C_{L}(\delta)$ is the non-dimensional lift coefficient. In order to simplify the expression of $L$, it is rewrote as

$$
L=k_{2} \delta
$$

with $k_{2}=\frac{\rho}{2} A_{R}\left(c_{1}-c_{2}|\delta|\right)\left|u_{R}\right| u_{R}$. Then $k=\operatorname{diag}\left\{T_{|n| n}, k_{2}\right\}$ acquired is regarded as the force coefficient matrix. Hence, the actuator forces and moments can be described as

$$
\begin{aligned}
\tau & =\left[\tau_{X}, \tau_{Y}, \tau_{N}\right]^{T}=B f\left(v_{r}, n, \delta\right)=B k u \\
& =\left[\begin{array}{cc}
1 & 0 \\
0 & 1 \\
-y_{n} & x_{\delta}
\end{array}\right]\left[\begin{array}{cc}
T_{|n| n} & 0 \\
0 & k_{2}
\end{array}\right]\left[\begin{array}{c}
|n| n \\
\delta
\end{array}\right]=\left[\begin{array}{c}
T_{|n| n}|n| n \\
k_{2} \delta \\
-y_{n} T_{|n| n}|n| n+x_{\delta} k_{2} \delta
\end{array}\right]
\end{aligned}
$$


For some kinds of ships, the force attack point of the thruster is located along with the longitudinal direction of the ship, which means $y_{n}=0$. Then the actuator forces and moments can be presented with the $Y_{\delta}$ replacing $k_{2}$ and $N_{\delta}$ for $x_{\delta} k_{2}$.

$$
\tau=\left[T_{|n| n}|n| n \quad Y_{\delta} \delta \quad N_{\delta} \delta\right]
$$

\subsection{Simplified Ship Dynamic Model}

The 3 DOF model in conjunction with expressions of actuator forces and moments is simplified to decoupled surge model and steering model due to the sway speed and yaw rate are usually much smaller compared to the cruise surge speed. As the first order Nomoto models are straightforward and simple response models describing the relationship between commanded rudder angle and heading movement, they are derived from the simplified steering model in this thesis for comparison studies.

\subsubsection{Surge Model}

Given that the yaw rate $(r)$ and sway velocity $(v)$ are small enough, and their effects on the surge motion are ignored, the speed dynamic model can be simplified to

$$
\left(m-X_{\dot{u}}\right) \dot{u}=X_{u} u+X_{|u| u}|u| u+X_{u u u} u^{3}+T_{|n| n}|n| n
$$

which is the second order modulus model with an extra third order term in the surge. The reason for adding the third order term is that it can show the best fit to the experimental data (Skjetne, Smogeli, and Fossen 2004). Considering the identifiability of the surge model, four inputs $\left(u,|u| u, u^{3}\right.$ and $\left.|n| n\right)$ in such model are not enough to identify all five parameters $\left(X_{u}, X_{|u| u}, X_{u u u}, T_{|n| n}\right.$ and $\left.X_{\dot{u}}\right)$. In other words, all hydrodynamic derivatives which define ship characteristics and its complete behaviors cannot be entirely estimated. Hence, equation (4.6) is reorganized into

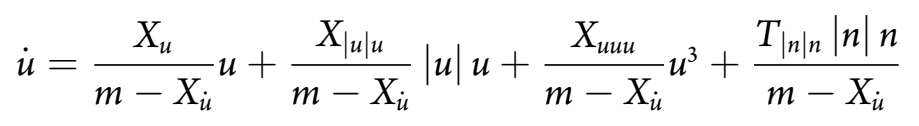

\subsubsection{Steering Model}

The remaining steering model 4.8 describing sway and yaw motions is obtained by decoupling the surge equation from (4.1). Here the resultant forward speed is calculated by $U=\sqrt{u_{\mathrm{o}}^{2}+r^{2}}$ based 
on the constant surge speed $\left(u_{\mathrm{o}}\right)$.

$$
\left[\begin{array}{cc}
m-Y_{\dot{v}} & m x_{g}-Y_{\dot{r}} \\
m x_{g}-N_{\dot{v}} & I_{z}-N_{\dot{r}}
\end{array}\right]\left[\begin{array}{c}
\dot{v} \\
\dot{r}
\end{array}\right]=\left[\begin{array}{ll}
n_{11} & n_{12} \\
n_{21} & n_{22}
\end{array}\right]\left[\begin{array}{l}
v \\
r
\end{array}\right]+\left[\begin{array}{c}
Y_{\delta} \\
N_{\delta}
\end{array}\right] \delta
$$

with

$$
\begin{aligned}
& n_{11}=-Y_{v}-Y_{|v| v}|v|-Y_{|r| v}|r| \\
& n_{12}=\left(m-X_{\dot{u}}\right) u_{\circ}-Y_{r}-Y_{|v| r}|v|-Y_{|r| r}|r| \\
& n_{21}=-\left(m-X_{\dot{u}}\right) u_{\circ}-N_{v}-N_{|v| v}|v|-N_{|r| v}|r| \\
& n_{22}=-N_{r}-N_{|v| r}|v|-N_{|r| r}|r|
\end{aligned}
$$

In terms of the identifiability of the model explained in surge model subsection, $(4.8)$ is modified into

$$
\begin{aligned}
\dot{v}= & \frac{I_{z}-N_{\dot{r}}}{\nabla}\left(\left(-Y_{v}-Y_{|v| v}|v|-Y_{|r| v}|r|\right) v+\left(\left(m-X_{\dot{u}}\right) u_{\mathrm{o}}-Y_{r}-\right.\right. \\
& \left.\left.Y_{|v| r}|v|-Y_{|r| r}|r|\right) r+Y_{\delta} \delta\right)-\frac{m x_{g}-Y_{\dot{r}}}{\nabla}\left(\left(-\left(m-X_{\dot{u}}\right) u_{\circ}-N_{v}-\right.\right. \\
& \left.\left.N_{|v| v}|v|-N_{|r| v}|r|\right) v+\left(-N_{r}-N_{|v| r}|v|-N_{|r| r}|r|\right) r+N_{\delta} \delta\right) \\
\dot{r}= & \frac{N_{\dot{v}}-m x_{g}}{\nabla}\left(\left(-Y_{v}-Y_{|v| v}|v|-Y_{|r| v}|r|\right) v+\left(\left(m-X_{\dot{u}}\right) u_{\circ}-Y_{r}-\right.\right. \\
& \left.\left.Y_{|v| r}|v|-Y_{|r| r}|r|\right) r+Y_{\delta} \delta\right)+\frac{m-Y_{\dot{v}}}{\nabla}\left(\left(-\left(m-X_{\dot{u}}\right) u_{\circ}-N_{v}-\right.\right. \\
& \left.\left.N_{|v| v}|v|-N_{|r| v}|r|\right) v+\left(-N_{r}-N_{|v| r}|v|-N_{|r| r}|r|\right) r+N_{\delta} \delta\right)
\end{aligned}
$$

with $\nabla=\left(m-Y_{\dot{v}}\right)\left(I_{z}-N_{\dot{r}}\right)-\left(m x_{g}-Y_{\dot{r}}\right)\left(m x_{g}-N_{\dot{v}}\right)$.

\subsubsection{Nomoto Models}

The first order linear Nomoto model is the most straightforward set describing the relationship between yaw rate and rudder angle, and its nonlinear extension appeared later as well. The first order linear Nomoto model can be derived from the linearized simplified steering model. Removing the the sway-yaw coupling terms from (4.8) gives the linear version of the simplified 
steering model expressed as

$$
\left[\begin{array}{cc}
m-Y_{\dot{v}} & m x_{g}-Y_{\dot{r}} \\
m x_{g}-N_{\dot{v}} & I_{z}-N_{\dot{r}}
\end{array}\right]\left[\begin{array}{c}
\dot{v} \\
\dot{r}
\end{array}\right]=\left[\begin{array}{cc}
Y_{v} & -\left(m-X_{\dot{u}}\right) u_{\mathrm{o}}+Y_{r} \\
\left(m-X_{\dot{u}}\right) u_{\mathrm{o}}+N_{v} & N_{r}
\end{array}\right]\left[\begin{array}{l}
v \\
r
\end{array}\right]+\left[\begin{array}{c}
Y_{\delta} \\
N_{\delta}
\end{array}\right] \delta
$$

Subsequently, a 1 DOF model so-called the second order Nomoto model is obtained by eliminating the sway speed from (4.11). This model is widely applied to design ship autopilot because of its compromise between simplicity and accuracy. Its time domain function is

$$
T_{1} T_{2} \ddot{r}+\left(T_{1}+T_{2}\right) \dot{r}+r=K \delta+K T_{3} \delta
$$

where the parameters calculated with the use of hydrodynamic coefficients are given as

$$
\begin{aligned}
T_{1} T_{2}= & \frac{\left(m-Y_{\dot{v}}\right)\left(I_{z}-N_{\dot{r}}\right)-\left(m x_{g}-Y_{\dot{r}}\right)\left(m x_{g}-N_{\dot{v}}\right)}{Y_{v} N_{r}+\left(\left(m-X_{\dot{u}}\right) u_{\mathrm{o}}-Y_{r}\right)\left(\left(m-X_{\dot{u}}\right) u_{\mathrm{o}}+N_{v}\right)} \\
T_{1}+T_{2}= & \frac{-Y_{v}\left(I_{z}-N_{\dot{r}}\right)-N_{r}\left(m-Y_{\dot{v}}\right)-\left(\left(m-X_{\dot{u}}\right) u_{\mathrm{o}}+Y_{r}\right)\left(m x_{g}-N_{\dot{v}}\right)}{Y_{v} N_{r}+\left(\left(m-X_{\dot{u}}\right) u_{\mathrm{o}}-Y_{r}\right)\left(\left(m-X_{\dot{u}}\right) u_{\mathrm{o}}+N_{v}\right)} \\
& +\frac{\left(\left(m-X_{\dot{u}}\right) u_{\mathrm{o}}+N_{v}\right)\left(m x_{g}-Y_{\dot{r}}\right)}{Y_{v} N_{r}+\left(\left(m-X_{\dot{u}}\right) u_{\mathrm{o}}-Y_{r}\right)\left(\left(m-X_{\dot{u}}\right) u_{\mathrm{o}}+N_{v}\right)} \\
T_{3}= & \frac{\left(m x_{g}-N_{\dot{v}}\right) Y_{\delta}-\left(m-Y_{\dot{v}}\right) N_{\delta}}{Y_{v} N_{r}+\left(\left(m-X_{\dot{u}}\right) u_{\mathrm{o}}-Y_{r}\right)\left(\left(m-X_{\dot{u}}\right) u_{\mathrm{o}}+N_{v}\right)}
\end{aligned}
$$

Equation (4.12) is further converted into the first order Nomoto model by setting the effective time constant satisfy the relationship $T=T_{1}+T_{2}-T_{3}$. The time domain function of the first order linear Nomoto model is illustrated as

$$
\operatorname{Tr}+r=K \delta
$$

of which the transfer function can also be obtained to explain the relationship between the heading angle and the rudder angle due to $r(s)=s \psi(s)$.

Based on the first order Nomoto model, Norrbin (Norrbin 1963) proposed the nonlinear version to make it applicable for the cases of big rudder defections.

$$
\begin{aligned}
\operatorname{Tr}+H_{N}(r) & =K \delta \\
H_{N}(r) & =n_{3} r^{3}+n_{2} r^{2}+n_{1} r+n_{\circ}
\end{aligned}
$$


which results in a common expression of the first order nonlinear Nomoto model as

$$
T \dot{r}+n_{3} r^{3}+n_{1} r=K \delta
$$

where $T$ is the steering time constant, $K$ is the rudder gain, $n_{3}$ is the coefficient of the nonlinear item, $n_{1}$ is a coefficient determined according to ship's stability of course, i.e., $n_{1}=1$ for a course-stable ship and $n_{1}=-1$ for a course-unstable ship. 


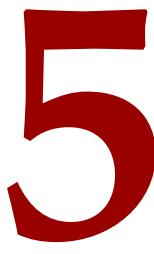

\section{ABC-LSSVR based Parameter Estimation Method}

To determine values of parameters in the ship dynamic model, several methods can be used. Instances are towing tank experiments, captive model experiments, computational fluid dynamics, and system identification combined with full-scale trails or free-running model tests. Among them, the last method is a sufficient and high cost-effective selection in estimating parameters of the ship dynamic model. When combined with full-scale trails, the scale effect due to the difference of Reynolds number between the full-scale ship and its model can be avoided. This reveals that the system identification technique is sufficient. When combined with free-running model tests, the system identification technique is a cost-effective solution due to many maneuvers can be easily generated once the first set of free-running model tests is carried out.

Various identification methods, e.g., ML, EKF, ANN, GA, PSO, SVM, are applicable for the parameter estimation of ship dynamic model, but the deficiencies existing in these methods require attention. Some methods are sensitive to the predetermined parameters, which are $\mathrm{ML}$, EKF. Some intelligent methods such as ANN, GA and PSO cannot always ensure global optimum for the model. Comparatively, the SVM based identification method avoids these deficiencies and can fulfill most requirements identified in the identification method. Besides, it can work 
beyond the limitations of the data acquisition of the vessel maneuvers due to it requires only a finite set of data. In fact, the experiment of the vessel carried out to stem data is expensive and restricted to various factors such as the weather condition, equipment, mobility of the vessel. So usually a limited number of measurements can be obtained for the identification. What's more, SVM based identification method can guarantee global optimal solutions because the optimization problem defined by it is a convex one typically quadratic programs (QP). This property makes a positive impact on the exactness of the identification results.

It is noticeable that the coefficients of variables in the regression model are sensitive to the structural parameters of SVM, such as the insensitivity factor, the regularization parameter, and kernel parameters, which implies that it is of high significance to assign these structural parameters with particular settings to guarantee good fitting of measurements. To determine these structural parameters particularly, methods such as cross-validation method, PSO, GA have been successfully studied by scholars. The cross-validation method is simple but time-consuming and at average accuracy. PSO and GA present the unavoidable problem of the local optimum. The $\mathrm{ABC}$ as one of the most recent nature-inspired algorithms has been proven to be a robust and efficient algorithm for solving global optimization problems over continuous space. It is also validated by some studies that the performance of $A B C$ is better than or similar to PSO and GA with the advantage of employing fewer control parameters. Therefore, the optimized SVM using $\mathrm{ABC}$ is proposed in this chapter for the parameter estimation of ship dynamic model. To date, the application of the hybrid approach of ABC and LS-SVR (which is one modified version of SVM as introduced in related work on SVM) to estimate parameters involved in the ship dynamic model has not been considered in the open literature.

Based on the previous introduction of LS-SVR in related work, the LS-SVR based parameter estimation method is illustrated in Section 5.1 where the samples are additionally constructed according to the structure of the estimation method. The optimization process of $A B C$ to determine a particular setting for the generalization parameter of LS-SVR is addressed in Section 5.2 in which the time complexity of the ABC-LSSVR method is also analyzed. The requirements, e.g., $\mathrm{R}_{5}-8$, are reflected in the process of proposing the ABC-LSSVR based parameter identification method in this chapter.

\subsection{LS-SVR based Parameter Estimation Method}

LS-SVR based system identification generally includes parameter identification and function estimation. Regarding parameter identification, the linear kernel function is adopted when 
identifying parameters of a linear system. For the nonlinear system, it is sufficient to use the polynomial kernel function. In the aspect of function estimation, the estimated function is always implicitly insinuated in the decision function which makes the explicit expression of the function very difficult. Fortunately, the first case, i.e., parameter identification by using LS-SVR, is the issue to be discussed.

\subsubsection{Introduction to The Method}

Parameter identification of linear systems. Suppose parameters of a linear system are required to be identified, and the formulae for the linear system is given by

$$
f(\mathbf{X})=\mathbf{A} \cdot \mathbf{X}
$$

where $\mathbf{A}$ is the vector of parameters. According to the decision function of (3.35), the above formulae is transferred into

$$
f(\mathbf{X})=\mathbf{A} \cdot \mathbf{X}=\sum_{i=1}^{l} a_{i} \mathbf{X}_{i} \cdot \mathbf{X}+b
$$

Assuming $b \rightarrow$ o gives the equality like

$$
\left(\mathbf{A}-\sum_{i=1}^{l} a_{i} \mathbf{X}_{i}\right) \cdot \mathbf{X}=0
$$

If the elements in $\mathbf{X}$ are linearly independent, then the parameters can be estimated by

$$
\mathbf{A}=\sum_{i=1}^{l} \alpha_{i} \mathbf{X}_{i}
$$

Parameter identification of nonlinear systems. Given a formula of a nonlinear system expressed as

$$
f(x)=a_{1} x^{2}+a_{2} x+a_{3}
$$


which is modified to the version of 5.6 by applying the quadratic polynomial kernel function.

$$
\begin{aligned}
f(x) & =a_{1} x^{2}+a_{2} x+a_{3} \\
& =\sum_{i=1}^{l} a_{i}\left(x_{i} x+d\right)^{2}+b \\
& =\sum_{i=1}^{l} a_{i} x_{i}^{2} x^{2}+2 d \sum_{i=1}^{l} a_{i} x_{i} x+d^{2} \sum_{i=1}^{l} a_{i}+b
\end{aligned}
$$

Consequently, the parameters can be computed by

$$
\begin{aligned}
& a_{1}=\sum_{i=1}^{l} a_{i} x_{i}^{2} \\
& a_{2}=2 d \sum_{i=1}^{l} a_{i} x_{i} \\
& a_{3}=d^{2} \sum_{i=1}^{l} a_{i}+b
\end{aligned}
$$

In the case of LS-SVR based parameter identification of either a linear or a nonlinear system, in particular, the nonlinear system, polynomial kernel function shows much superiority. But it is difficult to determine the value of $d$ and $p$ for the polynomial kernel function and the values of parameters are prone to be incorrect. Therefore, in the case of a known structure of the system which is linear with respect to the model parameters, the linear kernel function is preferable to be used instead of the polynomial kernel function. In this thesis, the linear kernel function is used in LS-SVR to identify parameters of ship dynamic models because the equations of ship dynamic models are linear with respect to the parameters.

\subsubsection{Construction of SAMples for IDENTIFication}

Inspired by (Sonnenburg and Woolsey 2013) about linearizing the drag speed model, one obtains the perturbation dynamics in the initial condition of the steady surge motion corresponding to the state and input $u=u_{\mathrm{o}}, v=\mathrm{o}, r=\mathrm{o}, \delta=\mathrm{o}$, and $n=n_{\mathrm{o}}$. The perturbation dynamic model of $(4.7)$ is expressed as

$$
\Delta \dot{u}=\frac{X_{u}+2 X_{|u| u}\left|u_{\mathrm{o}}\right|}{m-X_{\dot{u}}} \Delta u+\frac{X_{u u u}}{m-X_{\dot{u}}} \Delta u^{3}+\frac{2 T_{|n| n}\left|n_{\mathrm{o}}\right|}{m-X_{\dot{u}}} \Delta n
$$


with $\Delta u=u-u_{0}, \Delta n=n-n_{0}$.

According to the introduction of the LS-SVR based identification method, samples are obtained through the discretion of speed and steering models by the forward-difference approximation of Euler's stepping method. The discretized equations of surge mode and steering mode are given respectively as

$$
\begin{aligned}
\Delta u(k+1)= & \Delta u(k)+\frac{h\left(X_{u}+2 X_{|u| u} u_{\mathrm{o}}\right)}{m-X_{\dot{u}}} \Delta u(k)+\frac{h X_{u u u}}{m-X_{\dot{u}}} \Delta u^{3}(k) \\
& +\frac{2 h T_{|n| n}\left|n_{\mathrm{o}}\right|}{m-X_{\dot{u}}} \Delta n(k) \\
v(k+1)= & v(k)+\frac{h\left(I_{z}-N_{\dot{r}}\right)}{\nabla}\left(\left(Y_{v}+Y_{|v| v} v(k)+Y_{|r| v \mid}|r(k)|\right) v(k)\right. \\
& \left.+\left(-\left(m-X_{\dot{u}}\right) u_{\mathrm{o}}+Y_{r}+Y_{|v| r}|v(k)|+Y_{|r| r}|r(k)|\right) r(k)+Y_{\delta} \delta(k)\right) \\
& -\frac{h\left(m x_{g}-Y_{\dot{r}}\right)}{\nabla}\left(\left(\left(m-X_{\dot{u}}\right) u_{\mathrm{o}}+N_{v}+N_{|v| v}|v(k)|+N_{|r| v}|r(k)|\right)\right. \\
& \left.v(k)+\left(N_{r}+N_{|v| r \mid}|v(k)|+N_{|r| r}|r(k)|\right) r(k)+N_{\delta} \delta(k)\right) \\
r(k+1)= & r(k)+\frac{h\left(N_{\dot{v}}-m x_{g}\right)}{\nabla}\left(\left(Y_{v}+Y_{|v| v} v(k)+Y_{|r| v}|r(k)|\right) v(k)\right. \\
& \left.+\left(-\left(m-X_{\dot{u}}\right) u_{\mathrm{o}}+Y_{r}+Y_{|v| r}|v(k)|+Y_{|r| r}|r(k)|\right) r(k)+Y_{\delta} \delta(k)\right) \\
& +\frac{h\left(m-Y_{\dot{v}}\right)}{\nabla}\left(\left(\left(m-X_{\dot{u}}\right) u_{\mathrm{o}}+N_{v}+N_{|v| v \mid}|v(k)|+N_{|r| v \mid}|r(k)|\right)\right. \\
& \left.v(k)+\left(N_{r}+N_{|v| r \mid}|v(k)|+N_{|r| r}|r(k)|\right) r(k)+N_{\delta} \delta(k)\right)
\end{aligned}
$$

where $\nabla=\left(m-Y_{\dot{v}}\right)\left(I_{z}-N_{\dot{r}}\right)-\left(m x_{g}-Y_{\dot{r}}\right)\left(m x_{g}-N_{\dot{v}}\right), h$ is the time interval, $k+1$ and $k$ are two successive data. Subsequently, the input-output pairs are obtained. Inputs are expressed as

$$
\begin{aligned}
& \mathbf{X}_{\text {inp }}=\left[\Delta u(k), \Delta u^{3}(k), \Delta n(k)\right]_{3 \times 1}^{T} \\
& \mathbf{Y}_{\text {inp }}=[v(k),|v(k)| v(k),|r(k)| v(k), r(k),|v(k)| r(k),|r(k)| r(k), \delta(k)]_{7 \times 1}^{T} \\
& \mathbf{Z}_{\text {inp }}=[v(k),|v(k)| v(k),|r(k)| v(k), r(k),|v(k)| r(k),|r(k)| r(k), \delta(k)]_{7 \times_{1}}^{T} .
\end{aligned}
$$


Let

$$
\begin{aligned}
& \mathbf{A}=\left[\begin{array}{lll}
a_{1} & a_{2} & a_{3}
\end{array}\right]_{1 \times 3} \\
& \mathbf{B}=\left[\begin{array}{lllllll}
b_{1} & b_{2} & b_{3} & b_{4} & b_{5} & b_{6} & b_{7}
\end{array}\right]_{1 \times 7} \\
& \mathbf{C}=\left[\begin{array}{lllllll}
c_{1} & c_{2} & c_{3} & c_{4} & c_{5} & c_{6} & c_{7}
\end{array}\right]_{1 \times 7} \\
& a_{1}=1+\frac{h\left(X_{u}+2 X_{|u| u}\left|u_{0}\right|\right)}{m-X_{\dot{u}}} \\
& a_{2}=\frac{h X_{u u u}}{m-X_{\dot{u}}} \\
& a_{3}=\frac{2 h T_{|n| n}\left|n_{\mathrm{o}}\right|}{m-X_{\dot{u}}} \\
& b_{1}=1+\frac{\left(I_{z}-N_{\dot{r}}\right) Y_{v} h+\left(\left(m-X_{\dot{u}}\right) u_{\mathrm{o}}+N_{v}\right)\left(Y_{\dot{r}}-m x_{g}\right) h}{\nabla} \\
& b_{2}=\frac{\left(I_{z}-N_{\dot{r}}\right) Y_{|v| v} h-\left(m x_{g}-Y_{\dot{r}}\right) N_{|v| v} h}{\nabla} \\
& b_{3}=\frac{\left(I_{z}-N_{\dot{r}}\right) Y_{|r| v} h-\left(m x_{g}-Y_{\dot{r}}\right) N_{|r| v} h}{\nabla} \\
& b_{4}=\frac{\left(I_{z}-N_{\dot{r}}\right)\left(Y_{r}-\left(m-X_{\dot{u}}\right) u_{\mathrm{o}}\right) h-\left(m x_{g}-Y_{\dot{r}}\right) N_{r} h}{\nabla} \\
& b_{5}=\frac{\left(I_{z}-N_{\dot{r}}\right) Y_{|v| r} h-\left(m x_{g}-Y_{\dot{r}}\right) N_{|v| r} h}{\nabla} \\
& b_{6}=\frac{\left(I_{z}-N_{\dot{r}}\right) Y_{|r| r} h-\left(m x_{g}-Y_{\dot{r}}\right) N_{|r| r} h}{\nabla} \\
& b_{7}=\frac{\left(I_{z}-N_{\dot{r}}\right) Y_{\delta} h-\left(m x_{g}-Y_{\dot{r}}\right) N_{\delta} h}{\nabla} \\
& c_{1}=\frac{\left(N_{\dot{v}}-m x_{g}\right) Y_{v} h+\left(m-Y_{\dot{v}}\right)\left(\left(m-X_{\dot{u}}\right) u_{\mathrm{o}}+N_{v}\right) h}{\nabla} \\
& c_{2}=\frac{\left(N_{\dot{v}}-m x_{g}\right) Y_{|v| v} h+\left(m-Y_{\dot{v}}\right) N_{|v| v} h}{\nabla} \\
& c_{3}=\frac{\left(N_{\dot{v}}-m x_{g}\right) Y_{|r| v} h+\left(m-Y_{\dot{v}}\right) N_{|r| v} h}{\nabla} \\
& c_{4}=\mathbf{1}+\frac{\left(m-Y_{\dot{v}}\right) N_{r} h-\left(N_{\dot{v}}-m x_{g}\right)\left(\left(m-X_{\dot{u}}\right) u_{o}-Y_{r}\right) h}{\nabla} \\
& c_{5}=\frac{\left(N_{\dot{v}}-m x_{g}\right) Y_{|v| r} h+\left(m-Y_{\dot{v}}\right) N_{|v| r} h}{\nabla} \\
& c_{6}=\frac{\left(N_{\dot{v}}-m x_{g}\right) Y_{|r| r} h+\left(m-Y_{\dot{v}}\right) N_{|r| r} h}{\nabla}
\end{aligned}
$$




$$
c_{7}=\frac{\left(N_{\dot{v}}-m x_{g}\right) Y_{\delta} h+\left(m-Y_{\dot{v}}\right) N_{\delta} h}{\nabla}
$$

then the outputs are given by $\Delta u(k+1)=\mathbf{A} \mathbf{X}_{\text {inp }}, v(k+1)=\mathbf{B} \mathbf{Y}_{\text {inp }}, r(k+1)=\mathbf{C Z}_{\text {inp }}$.

\subsection{Optimization of LS-SVR Using ABC}

After the linear kernel function in LS-SVR is decided, the performance of LS-SVR strongly depends on the regularization parameter. Thus, the selection of the regularization parameter for LS-SVR is one of the most important steps when using it as an identifier.

$\mathrm{ABC}$ proposed by Karaboga in 2005 is one of the most recent nature-inspired algorithms based on foraging behaviors of bees, meanwhile, it has been proven to be a robust and efficient algorithm for solving global optimization problems over continuous space (Karaboga 2005). So, this paper uses $A B C$ to optimize the regularization parameter of LS-SVR and then applies LS-SVR with the optimized parameter to estimate parameters of the simplified dynamic model.

$\mathrm{ABC}$ consists of three groups of bees, i.e., employed bees, onlooker bees and scout bees, who play essential roles in completing the optimization procedure of $\mathrm{ABC}$. The employed bees are responsible for exploring new food source positions in their neighborhoods, evaluating the food quality (fitness value) of the new food sources, updating the current food sources, and sharing this information with onlooker bees waiting in hives. Onlooker bees choose a food source for exploration based on the information obtained from employed bees and update food sources using the same way as employed bees. If an employed bee cannot improve its food source quality within a predefined number of iterations ( Limit), it will become a scout bee. The scout bees will randomly find food sources within the search space. The details are explained as follows.

\subsubsection{Key Stages in Artificial Bee Colony Algorithm}

\subsubsection{INITIALIZATION}

The initial food sources randomly distributed within the search space are assigned to the employed bees. Every food source is an optimal solution, which includes information about the food position and food quality (fitness value). The food position is calculated by the following equation

$$
x_{i j}=x_{j}^{m i n}+a\left(x_{j}^{\max }-x_{j}^{m i n}\right),(i=1, \cdots, S, j=1, \cdots, D)
$$


where $x_{j}^{\max }$ and $x_{j}^{\min }$ are the lower and upper bounds of the $j$ th parameter respectively which decide the search space, $a$ is random number in range of $[0,1], S$ is the number of food sources which is usually equal to the number of the employed bees donated by $N P$ or the onlooker bees donated by $N P$, and $D$ is the dimension conformed by the number of optimization parameter (here is 1 due to the optimized parameter is the regularization parameter of LS-SVR). The fitness value $\left(\right.$ fitness $\left._{i}\right)$ is calculated by

$$
\text { fitness }_{i}=\frac{1}{1+\text { Obj. } f_{\cdot_{i}}}
$$

where $O b j f_{f_{i}}$ is the objective function of the ith solution, which can be expressed by

$$
\operatorname{Obj} f_{\cdot_{i}}=\frac{1}{N} \sum_{n=1}^{N}\left(y_{\text {act }}(n)-y_{\text {pre }}(n)\right)^{2}
$$

where $y_{a c t}$ are actual outputs, $y_{\text {pre }}$ are predicted outputs of identified models, $N$ is the number of samples.

\subsubsection{The Employed Bees Stage}

After initialization, employed bees start finding new food sources in their neighborhoods according to the following equation

$$
x_{i j}^{\text {new }}=x_{i j}+a\left(x_{i j}-x_{k j}\right),(i, k=1, \cdots, S, j=1, \cdots, D)
$$

where $x_{i j}^{n e w}$ is the $j$ th dimension of the new food source, $x_{k j}$ is the $j$ th dimension of $k$ th employed bee, $a$ is a random number restricted in $[-1,1], j$ th and $k$ th are randomly selected among initial solutions and are not equal to each other. The information of the ith new food source then is updated via (5.13) and (5.14). The selection of new food source is decided by the greedy selection mechanism, i.e., if the fitness value of the new food source is better than the previous one, the new food source will replace the previous one, and Limit is set to zero, otherwise, the new food source will be ignored and Limit is added by one. 


\subsubsection{The Onlooker Bees Stage}

The onlooker bees take food information from all employed bees. Every onlooker bee chooses a food source with a probability related to its fitness value. The probability is calculated by

$$
P_{i}=\frac{\text { fitness }_{i}}{\sum_{i=1}^{S} \text { fitness }_{i}}
$$

Obviously, the higher the fitness value the food source is, the better the food source is. In other words, the food source with high fitness value is much possible to be selected by onlooker bees. Then, the procedure of updating food sources used by employed bees is also applied to onlooker bees. If the fitness value of the new food source calculated for onlooker bees is better than employed bees, the employed bee is replaced by the onlooker bee.

\subsubsection{The Scout Bees Stage}

If an employed bee cannot improve its food source quality within a predefined number Limit, it will become a scout bee. The scout bees will randomly find food sources within the search space using (5.12).

\subsubsection{Procedure of ABC-LSSVR for IDENTIFICATION}

After obtaining the tuned regularization parameter of LS-SVR through ABC, LS-SVR is used to identify the parameters of the ship dynamic model. The cooperation of these procedures is called the ABC-LSSVR method which is depicted in Figure 5.2.1.

The steps of this method are described as follows:

Step 1: A set of data extracted from ship maneuvers, including the surge speed, the corresponding actual propeller shaft speed, the sway speed, the yaw rate and the related actual rudder angle, are handled to be the input-output pairs. The data such as surge speed, sway speed, and yaw rate are used as actual outputs to calculate the value of the objective function which is to provide the fitness value of the food source.

Step2: Apply the above-extracted data to tune the regularization parameter in LS-SVR by using $\mathrm{ABC}$. This step is summarized as follows:

Step2.1: Determine the parameters of $A B C$, such as the number of food sources $S$, the number of employed bees and onlooker bees $N P$, the maximum iteration $T$, and the special 


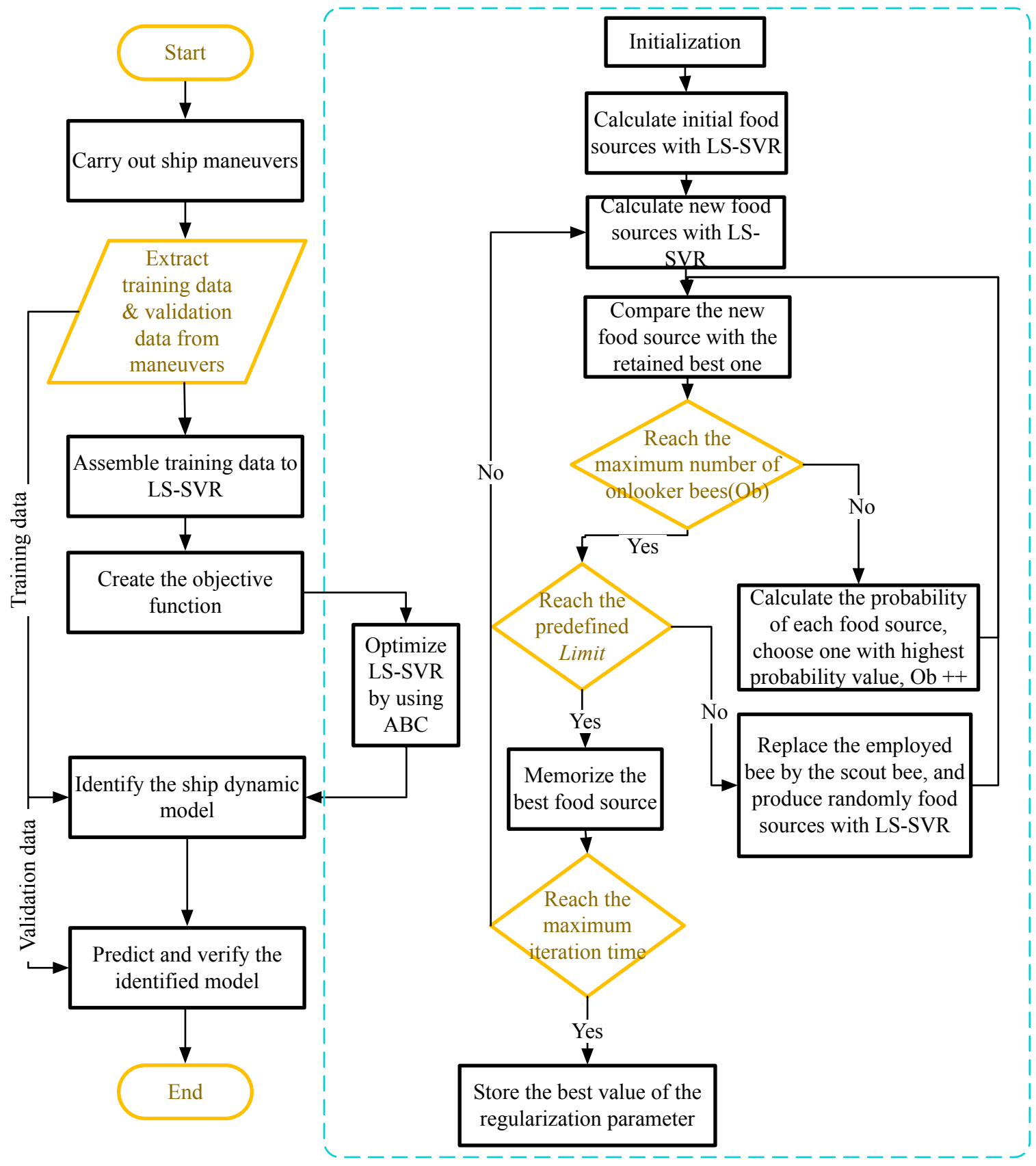

Figure 5.2.1: Graphical representation of the ABC-LSSVR.

number Limit, and finish the initialization (the first key steps in $A B C$ ).

Step 2.2: Search new food sources by employed bees using (5.15), update the information on the new food sources, and select the new solution by the greedy selection mechanism.

Step2.3: Onlooker bees get information from all employed bees and choose the food sources 
according to (5.16), and follow the same procedure as employed bees to update information of new food sources.

Step2.4: Compare the new food source with the retained best one, judge whether the current number of onlooker bees equals to the total number or not. If so, judge whether the number of mining the same food source is larger than Limit or not, otherwise, increase the number of onlooker bees and return to Step 2.3. If the number of mining the same food source reaches Limit, memorize the best food source, otherwise, produce new food sources and return to Step 2.3.

Step2.5: Calculate the current iteration number. If it reaches $T$, store the final result for the regularization parameter, otherwise, return to Step $\mathbf{2 . 2}$.

Step3: Apply the above-extracted data and result of Step 2.5 to LS-SVR in combination with the ship dynamic model, then get the identified model.

Step4: Substitute the above-extracted actual propeller shaft speed and actual rudder angle to the identified ship dynamic models to generate some predictions such as surge speed, sway speed and yaw rate, and then compare these predictions with the extracted outputs which are not used for identification, respectively.

\subsubsection{COMPlexity ANalysis}

In this subsection, the complexity mainly concerning the time complexity of the proposed ABC-LSSVR identification method is discussed. The proposed method firstly optimizes the regularization parameter of LS-SVR with the use of $A B C$, and then applies the optimized LS-SVR to estimate the parameters of the ship dynamic model. So, the computational cost of the $A B C-L S S V R$ method is the summation of the time complexity of $A B C$ and the time complexity of LS-SVR.

The time complexity of $\mathrm{ABC}$ is major defined by five parts including the initialization, the search operation of employed bees, the calculation of the probability of food sources, the search operation of onlooker bees, and the search operation of scout bees. According to the prementioned optimization procedure of $\mathrm{ABC}$, the computational cost of these five parts are $O(S N), O\left(S\left(N+N^{2}\right)\right), O(S), O\left(S\left(S+\left(N+N^{2}\right)+N\right)\right)$, and $O(S N)$, respectively. Therefore, the overall time complexity of $\mathrm{ABC}$ is $\mathrm{O}\left(S N+T_{i t}\left(S N+S\left(S+N+N^{2}\right)\right)\right)$ where $S$ is the number of food sources, $N$ is the number of samples selected for optimization, $T_{i t}$ is the number of iterations. Furthermore, the generic expression of the time complexity of $\mathrm{ABC}$ is $O\left(N^{2}\right)$. 
For SVM, the time complexity depends on solving the convex quadratic programming problem and the dual optimization, which is $O\left(\ln _{s v}^{2}+n_{s v}^{3}\right)$. Here $l$ is the number of patterns, $n_{s v}$ is the number of support vectors. Since LS-SVR is developed by Sukyens (Suykens and Vandewalle 1999) on the basis of the discussion of How much can the SVM formulation be simplified without losing any of its advantages? in which the equality constraints replace the inequality constraints of SVM, the convex quadratic programming problem of it is converted into the linear equations. However, all patterns are treated as the support vectors in LS-SVR, which implies that $l=n_{s v}$. Therefore, the overall time complexity of LS-SVR is given as $O\left(l^{3}\right)$. As seen, the overall time complexity of LS-SVR might increase drastically with the increase of the patterns adopted for identification. In the worst case, the pattern dataset should be not more than a couple of 10000 samples.

Consequently, one can conclude that the overall time complexity of the proposed identification method is $\mathrm{O}\left(\mathrm{N}^{2}+l^{3}\right)$. 


\section{6 Implementation of the SIMSD Prototype}

The proposed simplified ship dynamic model and estimation method should be evaluated through a complete implementation. Trough the evaluation results, the degree of requirements' fulfillment can be investigated. In this chapter, the architecture of the developed system identification-based modeling of ship dynamics (SIMSD) is designed in Section 6.1 and the implementation of the prototype on the computer in MATLAB environment is introduced in Section 6.2.

\subsection{SIMSD System Design}

To evaluate the proposed solution in modeling ship dynamics, a software prototype so-called SIMSD is created as shown in Figure 6.1.1 where the primary components and interactions between them are presented. The components including observed data, ship dynamic model, identification method, and validation are determined according to the procedure of system identification in identify ship dynamic model. Each component is implemented by using relevant functions and files built in MATLAB. This way leads to a modular design of the implementation of the estimation of the ship dynamic model based on the system identification technique. It is 
additionally convenient to change every modular according to the requirement.

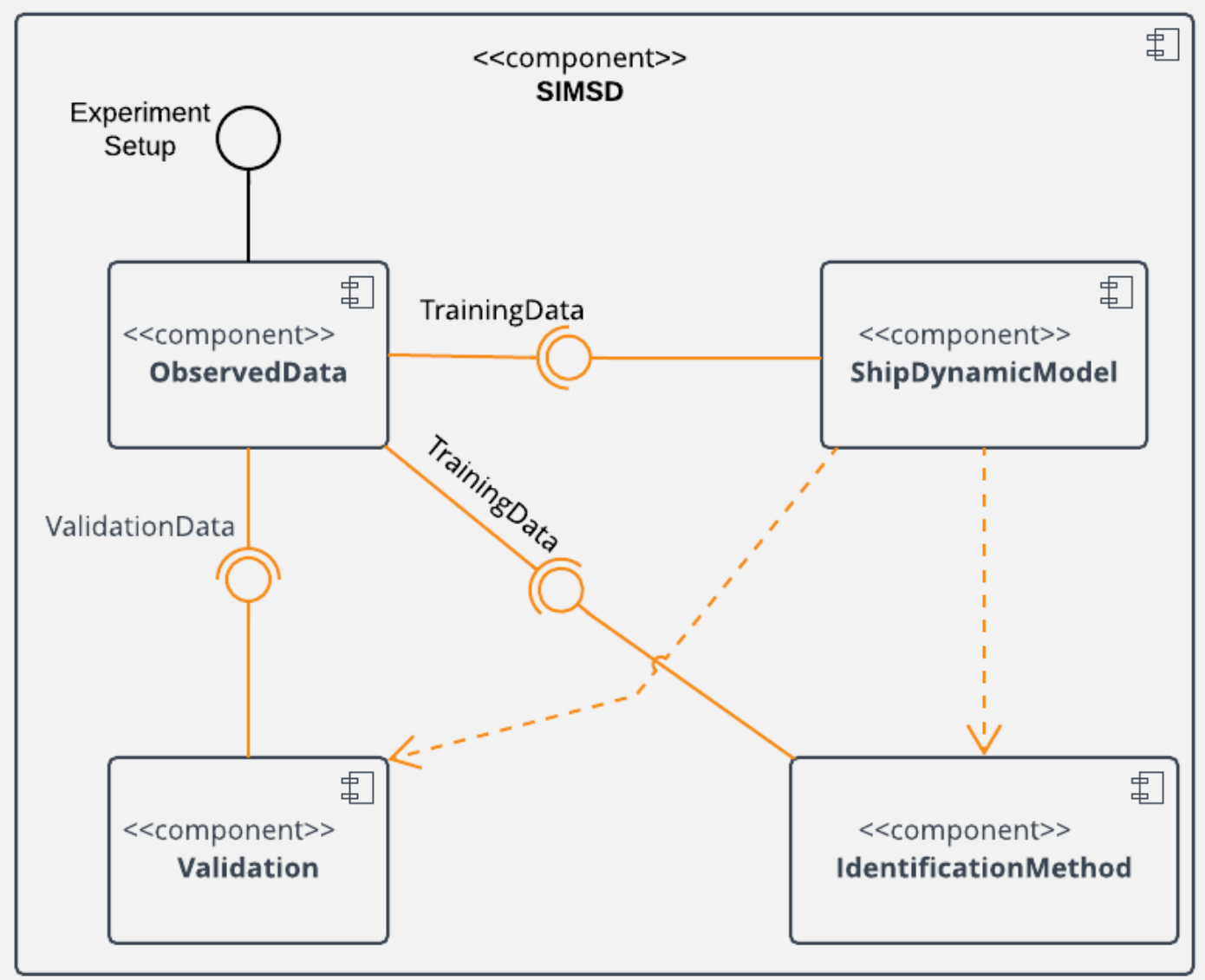

Figure 6.1.1: Component diagram of the SIMSD system.

\subsection{IMPLEMENTATION}

Snapshots are taken from the folder to present the files and functions that are implementing the prototype, see Figure 6.2.1. The files and functions presented are limited to important core functionality. Two classes of data including simulated data and experimental data are stored as the observed data. The simulated data are generated by using a high reality model with predetermined parameters of a full-scale ship, which is implemented directly in MATLAB. The experimental data stored in an Excel spreadsheet file are extracted from measurements recorded by sensors. The observed data are divided into two groups, i.e., training data and validation data. 
The training data are then read through the built-in function $x l s r e a d()$ and transferred to the function of the ship dynamic model. Meanwhile, with the application of the function of the identification method, the parameters involved in the model are estimated. Before determining the values of these parameters, the validity of the identification results shall be verified through the validation process. In this process, the inputs in the validation data, e.g., a time series of commanded rudder angle, are delivered to the identified model to generate the relevant output which is compared with the actual output stemmed from the validation data to get its MSE. If the MSE is not greater than the pre-set number, the identified model is accepted, and the values of parameters will be shown in the command window of MATLAB, otherwise, 'failed' will appear. 


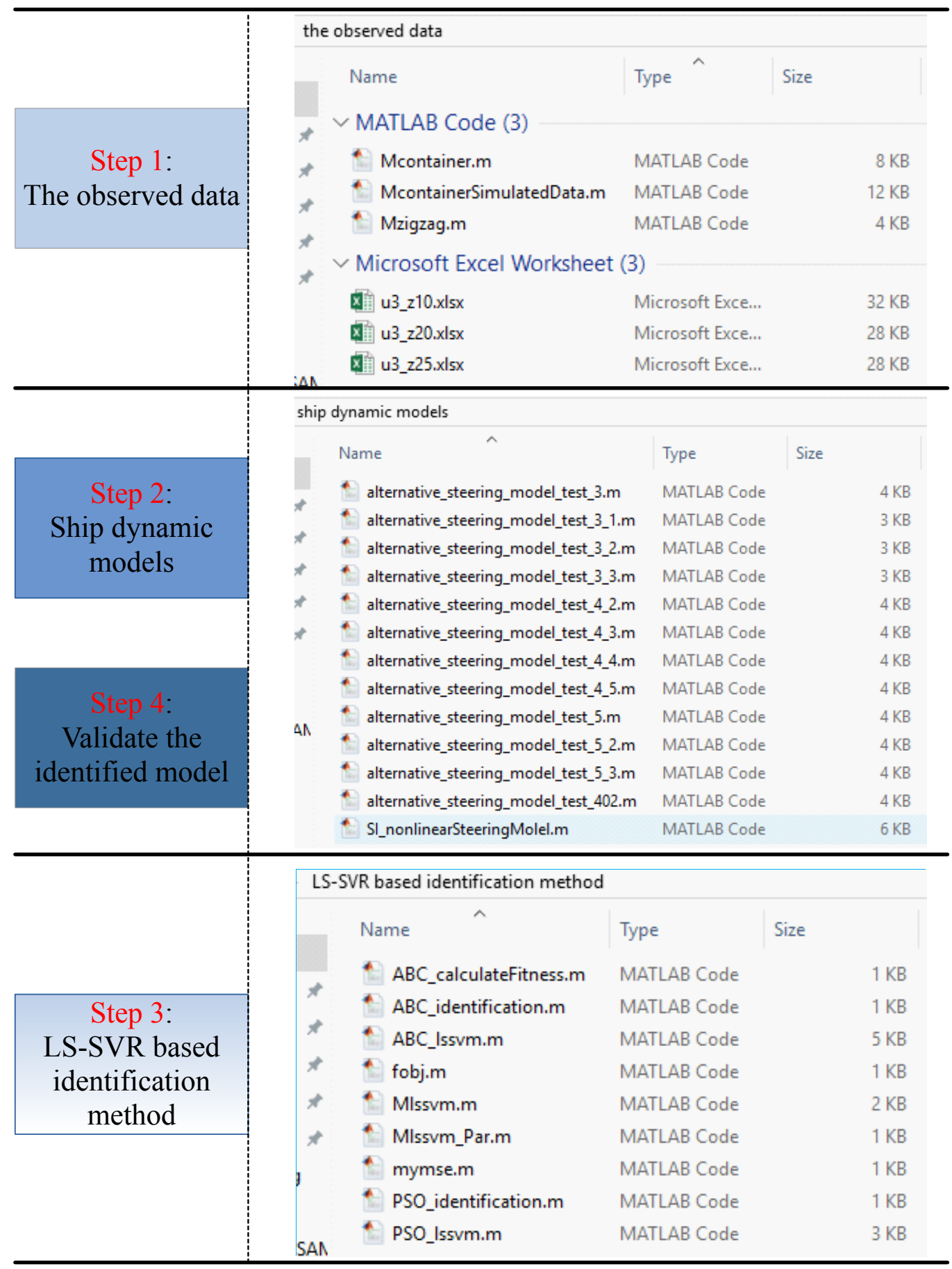

Figure 6.2.1: Snapshots of functions and files used for SIMSD implementation. 


\section{7 \\ Evaluation}

With the application of the previously created an implementation, the proposed solution on optimized identification method-based modeling of ship dynamics can be evaluated in this chapter through special case studies. Four major case studies are carried out for different purposes which are presented in Section 7.1. The first case study on comparing the commonly used LS with LS-SVR for identifying Nomoto models is to demonstrate that the LS-SVR based identification method is a powerful selection. Unlike LS-SVR based identification method which requires no initial values of model parameters and works with finite data, RLS needs to know the initial parameter values that make a significant impact on the identification results. In case study 2, RLS benefits from LS-SVR to identify a steering model based on the initial values of parameters obtained firstly by using LS-SVR. The effectiveness of the proposed ABC-LSSVR method is validated through case study 3 which further leads to special considerations on further simplification of the simplified steering dynamic model. Afterward, the further simplified dynamic model is validated through case study 4 on the I-Nav-II vessel. In the end, the overall results of the case studies are presented and discussed to conclude the answers to the research question. Meanwhile, the degree of fulfillment of requirements is judged. 


\subsection{Design of Case Studies}

Several case studies for particular goals are carried out before the finally experimental study on testing the simplified ship dynamic model by using the optimized LS-SVR. Cases including case number, purpose and method applied to achieve the purpose are depicted in detail as Table 7.1.1 presented.

Table 7.1.1: Description of the case studies.

\begin{tabular}{|c|c|c|c|}
\hline $\begin{array}{l}\text { Case } \\
\text { study }\end{array}$ & Method & Ship dynamic model & Purpose \\
\hline 1 & $\begin{array}{l}\text { Compare the commonly- } \\
\text { used LS with LS-SVR. }\end{array}$ & $\begin{array}{l}\text { The first order linear } \\
\text { and nonlinear Nomoto } \\
\text { models. }\end{array}$ & $\begin{array}{l}\text { Decide a robust parame- } \\
\text { ter estimation method. }\end{array}$ \\
\hline 2 & $\begin{array}{l}\text { Apply LS-SVR to supply RLS } \\
\text { initial parameter values with } \\
\text { the use of finite data. }\end{array}$ & $\begin{array}{l}\text { The linear steering } \\
\text { model. }\end{array}$ & $\begin{array}{l}\text { Present the advantages } \\
\text { of LS-SVR. }\end{array}$ \\
\hline 3 & $\begin{array}{l}\text { Optimize parameters in LS- } \\
\text { SVR by using ABC, CV, and } \\
\text { PSO, respectively, then com- } \\
\text { pare the results. Use the op- } \\
\text { timized LS-SVR to identify } \\
\text { the simplified ship dynamic } \\
\text { model. }\end{array}$ & $\begin{array}{l}\text { The } 4 \text { DOF dynamic } \\
\text { model of a large } \\
\text { container ship with pre- } \\
\text { determined parameters, } \\
\text { and the simplified ship } \\
\text { dynamic model. }\end{array}$ & $\begin{array}{l}\text { Verify the preferable } \\
\text { performance of } A B C \text { on } \\
\text { optimizing parameters } \\
\text { in LS-SVR and acquire } \\
\text { parameters of the sim- } \\
\text { plified ship dynamic } \\
\text { model. }\end{array}$ \\
\hline 4 & $\begin{array}{l}\text { Identify a simplified dynamic } \\
\text { model for a real ship, so- } \\
\text { called I-Nav-II vessel, by us- } \\
\text { ing data stemmed from full- } \\
\text { scale trails and the optimized } \\
\text { LS-SVR. }\end{array}$ & $\begin{array}{l}\text { The further simplified } \\
\text { steering dynamic model } \\
\text { obtained from case } \\
\text { study } 3 \text {. }\end{array}$ & $\begin{array}{l}\text { Demonstrate the fur- } \\
\text { ther simplified dynamic } \\
\text { model is valid to de- } \\
\text { scribe dynamics of } \\
\text { different ships. }\end{array}$ \\
\hline
\end{tabular}

\subsection{CASE 1: Selection and Comparison between LS ANd LS-SVR}

LS-SVR based identification method is indicated to be a prior selection for identification based on the qualitative analysis of identification methods in related work part on the identification of ship dynamic models. To quantitatively present this point, one of the comparison studies among identification methods is carried out between LS and LS-SVR. 


\subsubsection{Data Processing}

A standard maneuver for ship model identification is the zigzag maneuver that is also defined by an IMO resolution A 751(18) and by given hints in IMO MSC Circ. 1053 to be executed in sea trials for ships. Although the IMO documents mainly for-see $10^{\circ} / 10^{\circ}$ or $20^{\circ} / 20^{\circ}$ zigzag maneuver and for special cases known as very small zigzag maneuvers (VSZZ) such as $5^{\circ} / 5^{\circ}$, a set of a flexible set of zigzag angles are also allowable. The data used for training and validating parameter estimation of ship response models are obtained from the zigzag maneuvering experiments of a small ship. Two groups of zigzag experiments containing $20^{\circ} / 20^{\circ}$ zigzag for estimating parameters and $30^{\circ} / 30^{\circ}$ zigzag for validation are derived with initial states including the rudder angle of $0^{\circ}$, the yaw rate of $0^{\circ} / \mathrm{s}$, and sway speed of $\mathrm{m} / \mathrm{s}$. In the $20^{\circ} / 20^{\circ}$ zigzag test, the forward speed is 1.5 $\mathrm{m} / \mathrm{s}$, the sampling time is $82 \mathrm{~s}$, the interval is $1 \mathrm{~s}$, and the initial heading angle is $46.71^{\circ}$. In the $30^{\circ} / 30^{\circ}$ zigzag test, the corresponding values are $1.7 \mathrm{~m} / \mathrm{s}, 89 \mathrm{~s}, 1 \mathrm{~s}$, and $78.28^{\circ}$, respectively. The time series of the rudder angle and heading angle are shown in Figure 7.2.1.

\subsubsection{Results ANd Analysis}

The estimated maneuvering indices are shown in Table 7.2.1. The comparisons of $K$ and $T$ estimated by LS and LS-SVR between the first order linear and the nonlinear Nomoto models indicate that the two structures of the response model are coincided mathematically, which confirms the logic sense that the two different categories of response models reflect the same dynamics of the ship investigated.

Table 7.2.1: Identified maneuvering indices of the first order Nomoto models.

\begin{tabular}{llllll}
\hline Method & \multicolumn{2}{c}{ Linear } & \multicolumn{3}{l}{ Nonlinear } \\
\hline \multirow{2}{*}{ LS } & $\mathrm{K}$ & $\mathrm{T}$ & $\mathrm{K}$ & $\mathrm{T}$ & $\alpha$ \\
LS-SVR & 0.1960 & 1.1657 & 0.1933 & 1.1275 & -0.0006 \\
\hline
\end{tabular}

Once the model is well defined, the maneuvering prediction using the identified response model should be carried out to verify the accuracy of estimated parameters. In Figure 7.2.2, the prediction results of the identified first order linear Nomoto models are shown. The statistical validation of the prediction results for the two identification methods is presented in Figure 7.2.3. For the identified first order nonlinear Nomoto model, these results are demonstrated in 

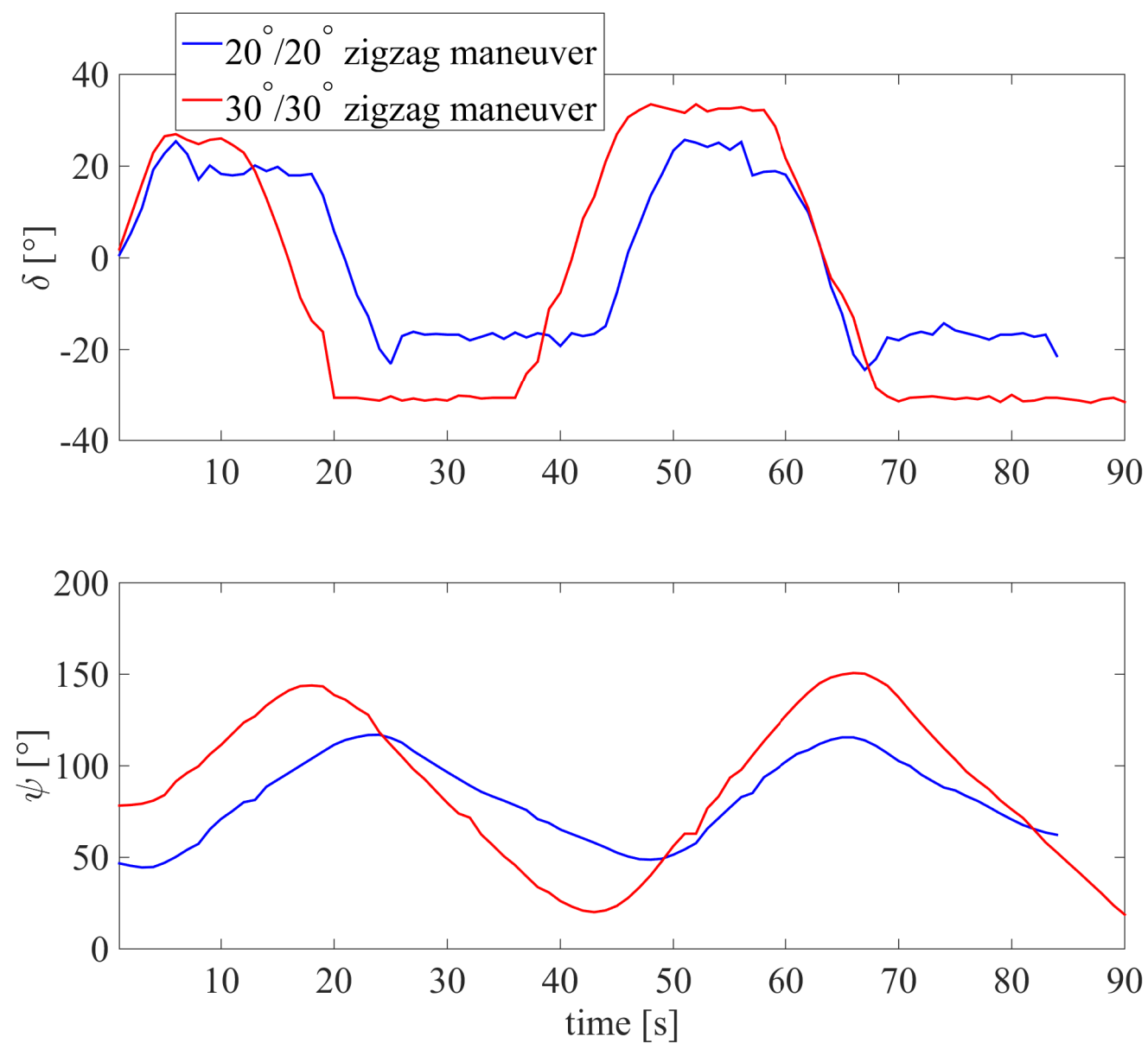

Figure 7.2.1: Time series of the rudder and heading angles. The blue lines are the actual rudder angle in the upper sub-figure of the $20^{\circ} / 20^{\circ}$ zigzag maneuver, and the heading angle in the bottom sub-figure of the $20^{\circ} / 20^{\circ}$ zigzag maneuver. The red lines are the actual rudder angle in the upper sub-figure of the $30^{\circ} / 30^{\circ}$ zigzag maneuver, and the heading angle in the bottom sub-figure of the $30^{\circ} / 30^{\circ}$ zigzag maneuver.

Figure 7.2.4 and Figure 7.2.5, respectively.

From the prediction results, we can see that both the first order linear and nonlinear Nomoto models can be estimated by LS and LS-SVR. Additionally, it can be found in Figure 7.2.3 that the standard derivation of the histogram of LS-SVR is smaller than that of LS whose cross-correlation is slightly better except for the auto-correlation. Figure 7.2.5 indicates that the standard derivation of the histogram of LS-SVR is close to that of LS. Therefore, the performance of LS-SVR is a little 


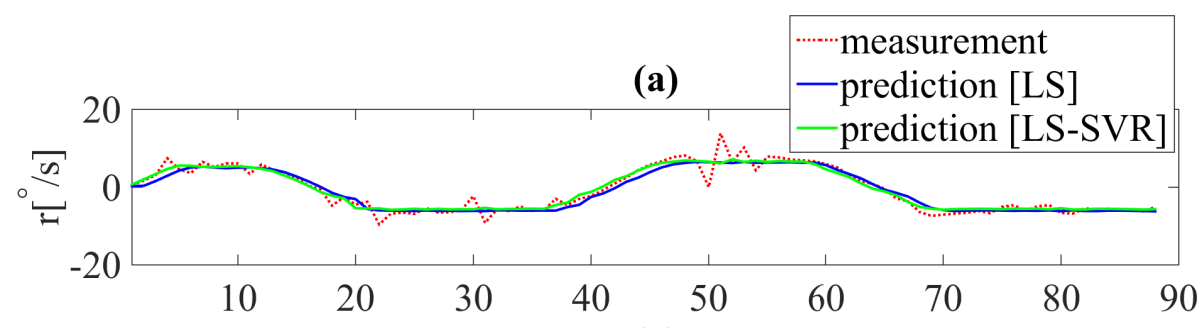

(b)
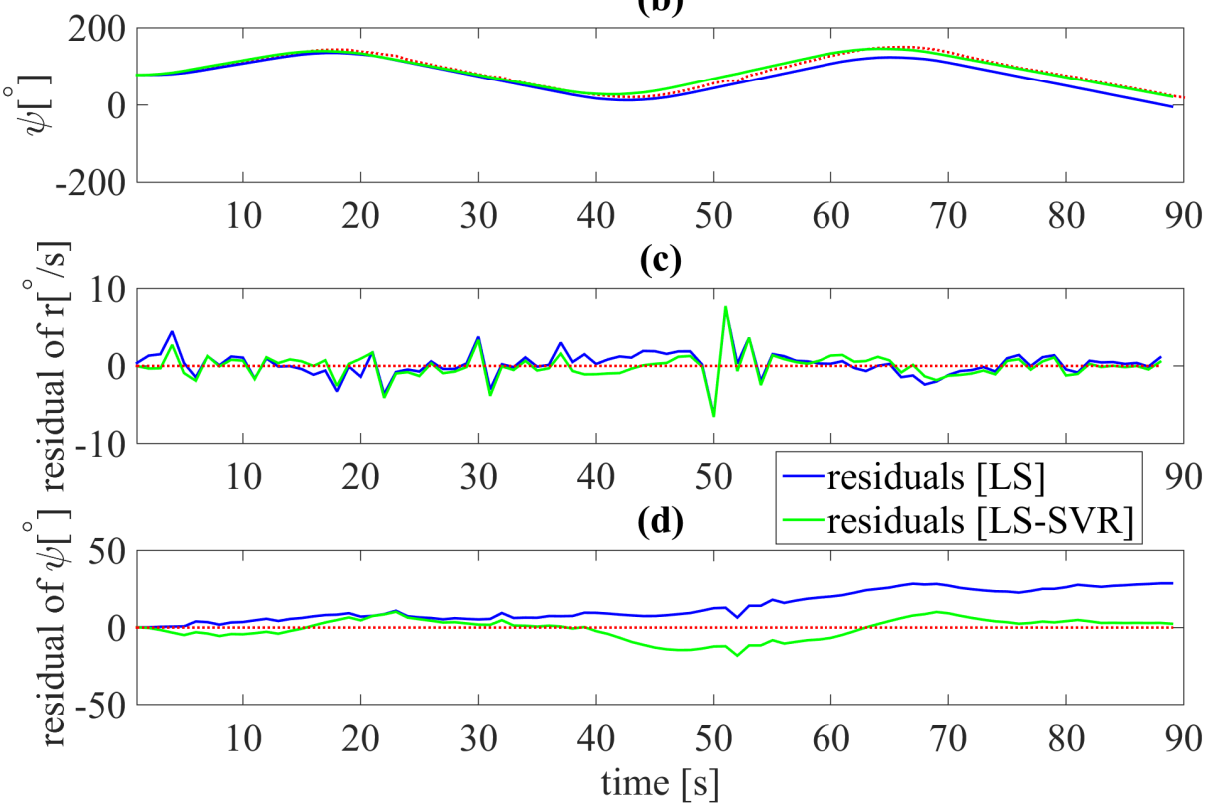

Figure 7.2.2: Prediction results of the first order linear models identified by LS and LS-SVR, respectively. (a) measured yaw rate and predicted yaw rate, (b) measured heading angle and predicted heading angle, (c) residual of the yaw rate by calculating the difference between measurements and predictions, (d) residual of the heading angle obtained by calculating the difference between measurements and predictions.

better than LS for identifying ship response models.

For these results, several aspects can be explained as follows. LS aims at minimizing a squared norm. This means that if an outlier is existing in the training data, the outlier can adversely affect the identification results. In fact, the data used for identification are experimental maneuvering data of a small ship, which are corrupted by color noises from measurement noises, sensor underlying noises, and environmental disturbances. So, the outliers caused by the color noises influence the identification results of LS. On the contrary, LS-SVR simultaneously achieves both structural risk minimization and empirical risk minimization when estimating parameters of the response model. This characteristic of LS-SVR sufficiently decreases its sensitivity to the outlier. 

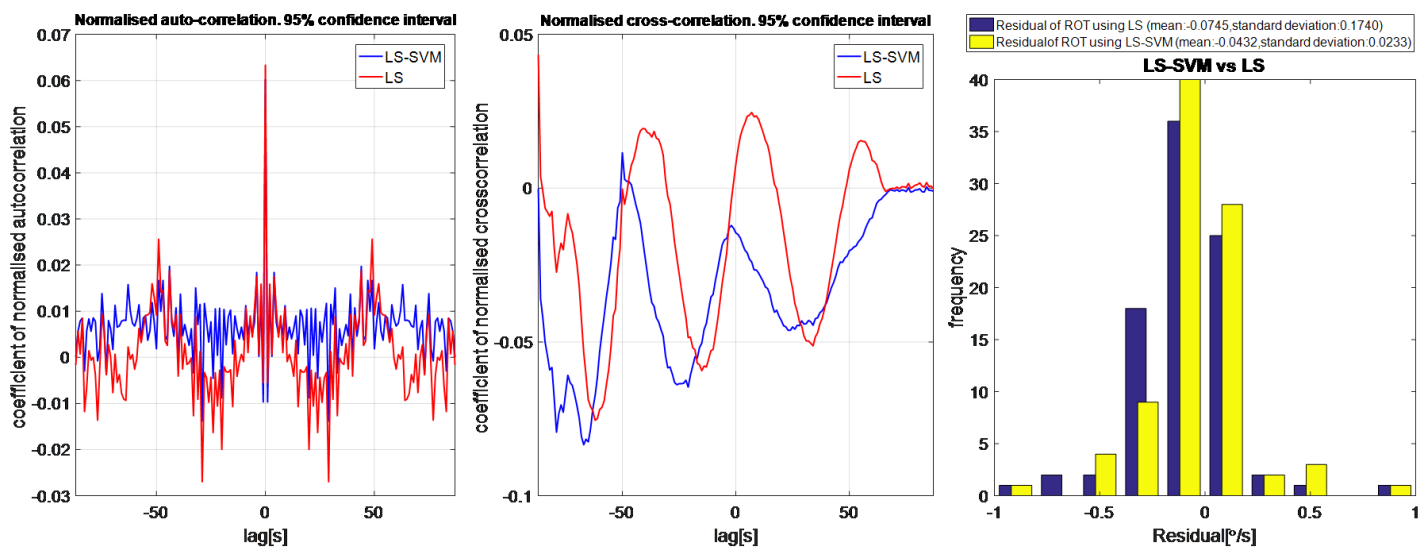

Figure 7.2.3: Statistical validation of the identified first order linear models. The left subfigure is the normalized auto-correlation, the middle one is the normalized cross-correlation, the right one presents the residual analysis.

Therefore, for the cases studied here, LS-SVR can present better identification performance and obtain more accurate identified parameters than LS.

\subsection{CASE 2: Improvement OF RLS Using LS-SVR}

Since the change of current weather and ship loading conditions may cause parameter variations of ship maneuvering models, the well-known RLS with an advantage of simple construction can be used to complete this task. As well known, the identification results of RLS are sensitive to the initial values of parameters (Zhang and Zou 2013). Hence, this thesis tries to conquer such drawback of RLS by benefiting from LS-SVR which is a kind of batch identification technique and requires no initial estimation of the parameters, to predetermine the values of parameters.

\subsubsection{Data Processing}

A linear steering model of a Merchant ship Mariner class presented in Appendix $C$ is the model needed to be identified. Meanwhile, the model with existing parameters learned from the study in (Åström and Källström 1976) is synergistically employed to generate data used for learning and validation of the parameter estimation. Particulars of the ship are shown in Table 7.3.1.

Two groups of zigzag maneuver including $20^{\circ} / 20^{\circ}$ zigzag maneuver for estimation and $10^{\circ} / 10^{\circ}$ zigzag maneuver for validation are simulated under the initial states involving the forward speed of $7.7 \mathrm{~m} / \mathrm{s}$, the rudder angle of $0^{\circ}$, the heading angle of $0^{\circ}$, the yaw rate of $0^{\circ} / \mathrm{s}$, and the sway 


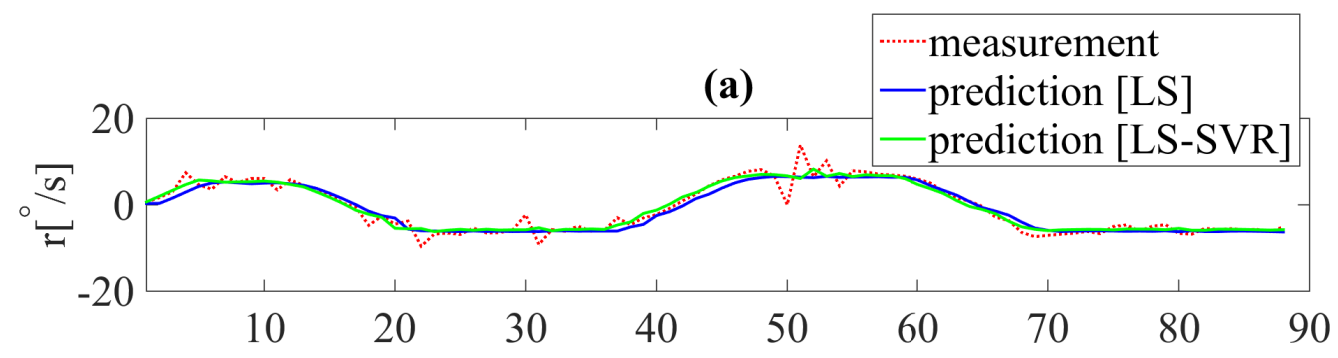

(b)
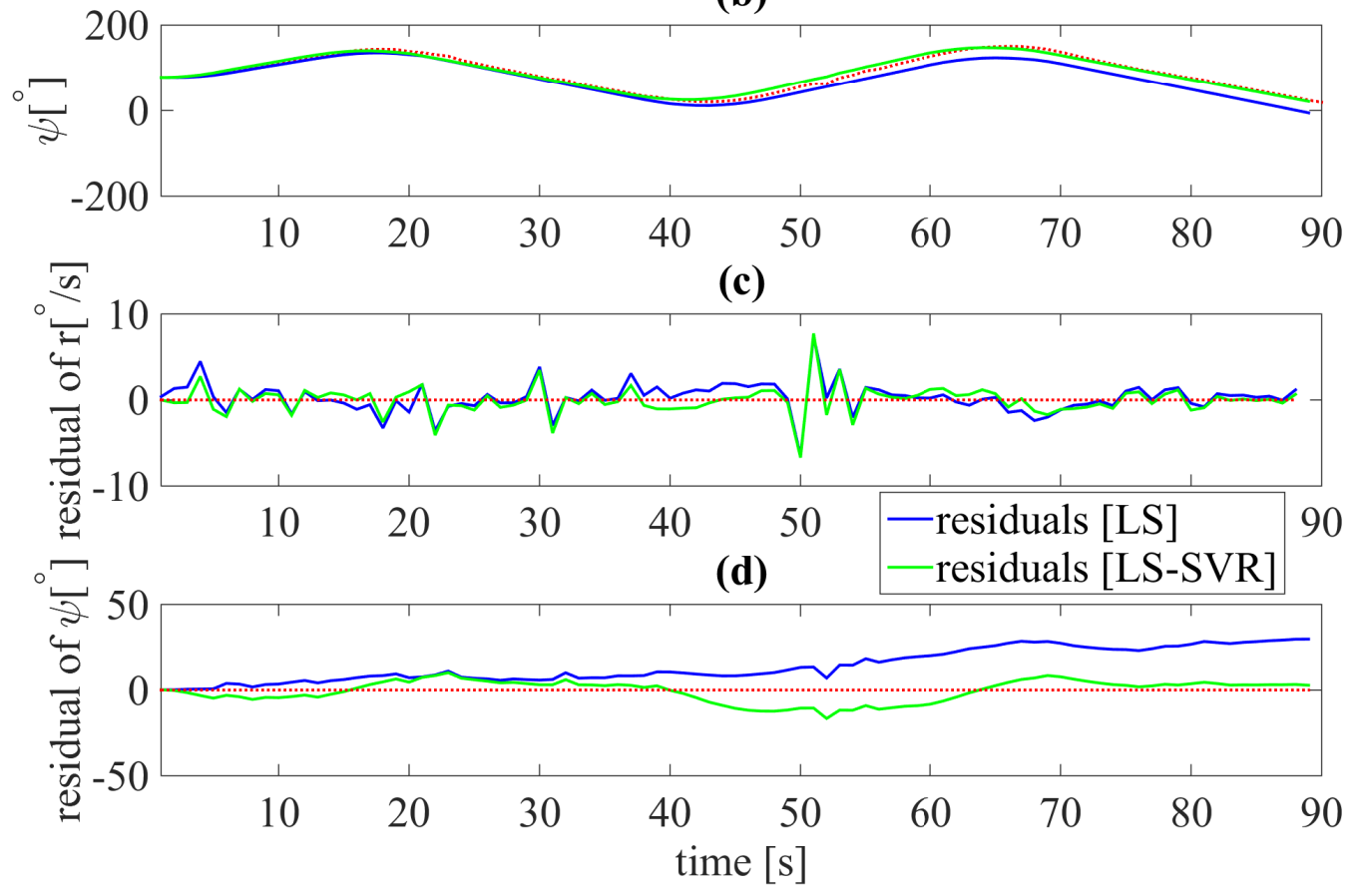

Figure 7.2.4: Prediction results of the first order nonlinear models identified by LS and LSSVR, respectively. (a) measured yaw rate and predicted yaw rate, (b) measured heading angle and predicted heading angle, (c) residual of the yaw rate obtained by calculating the difference between measurements and predictions, (d) residual of the heading angle by calculating the difference between measurements and predictions.

Table 7.3.1: Parameters of the Merchant Ship Mariner class.

\begin{tabular}{cccc}
\hline Length $(L, m)$ & 161 & Speed $(U, m / s)$ & 7.7 \\
\hline$a_{11}$ & -0.693 & $a_{21}$ & -3.41 \\
$a_{12}$ & -0.304 & $a_{22}$ & -2.17 \\
$b_{11}$ & 0.207 & $b_{21}$ & -1.63 \\
\hline
\end{tabular}



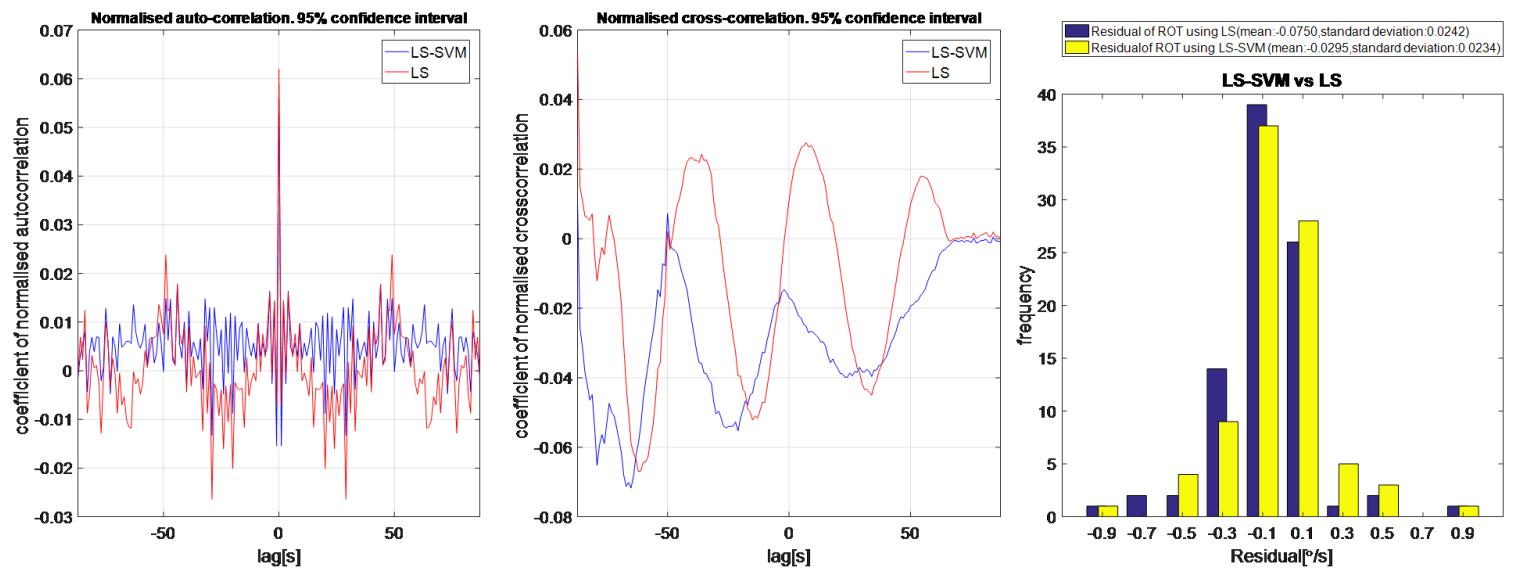

Figure 7.2.5: Statistical validation of the identified first order nonlinear models. The left sub-figure is the normalized auto-correlation, the middle one is the normalized crosscorrelation, the right one presents the residual analysis.

speed of o m/s. The sampling time is 500s, and the interval is $0.5 \mathrm{~s} .1000$ measurement pairs of $v, r$, $\delta$, and resultant speed $U$ are recorded for parameter estimation of the steering model. The original simulation data are illustrated in Figure 7.3.1. The commanded rudder angles in-between 200 s to 220 are corrupted by the noises of the level of $1.5 \%$ commanded rudder angle. The so-called noised simulation data are also depicted in Figure 7.3.1.

\subsubsection{SELEction OF SAMPLES For LS-SVR}

LS-SVR as a batch technique avoids lengthy iteration and needs no initial estimation of parameters. However, one can see that the first and significant step in applying LS-SVR is to choose an appropriate number of samples. The solution for such a problem is proposed by comprehensively analyzing the convergence of LS-SVR and the accuracy of the estimated parameters in the condition of varying number and interval of samples. The samples are selected from the noised maneuvering data. The number of samples switches from 10 to 1000 in steps of 10. For each number of samples, the interval changes from 1 to 10.

To investigate the performance of LS-SVR, identification results shown in Figure 7.3.2 are compared with the true values listed in Table 7.3.1. As the former 400 samples are not corrupted by the noises, it can be observed that the selection of 200 samples without noise in all intervals can satisfy the LS-SVR achievement of identification. But for noised data, only samples in the interval of 3,6 , and 9 can guarantee every estimated parameter matching well with its true value. 

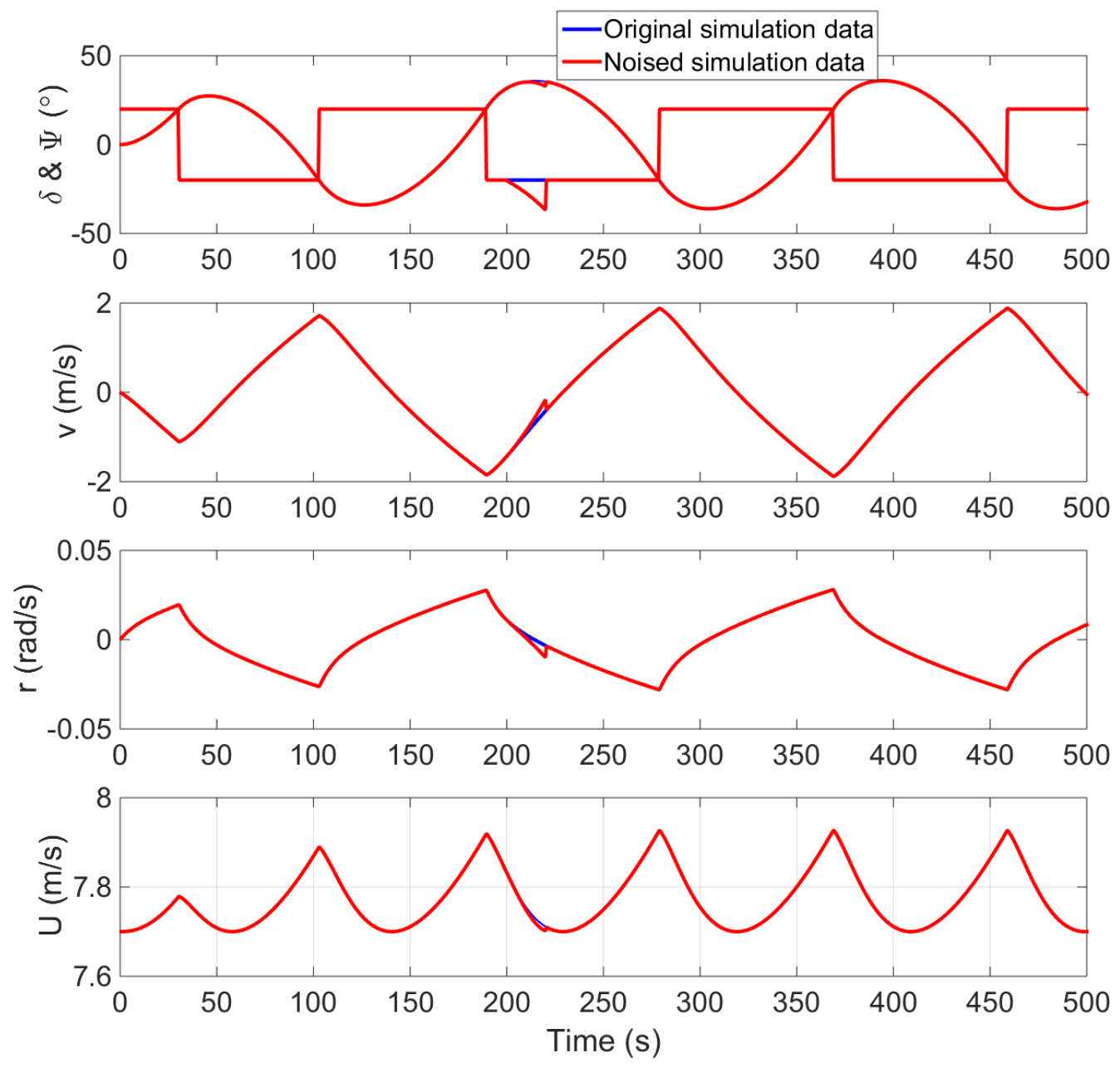

Figure 7.3.1: Simulation of the $20^{\circ} / 20^{\circ}$ zigzag maneuver. The top sub-figure is the commanded rudder and the heading angles, the second one is the sway velocity, the third one is the yaw rate, the bottom is the resultant speed.

Finally, 200 samples in the interval of 3 are sufficient to be used by LS-SVR to provide initial values of parameters for RLS.

\subsubsection{IDENTIFication Results}

It is worthy to claim that more than three parameters cannot converge to true values while 1000 samples are utilized by RLS for identification. Hence, the simulation time of $20^{\circ} / 20^{\circ}$ zigzag maneuver is increased to 1 ooos. The final identification results of the linear steering model by 

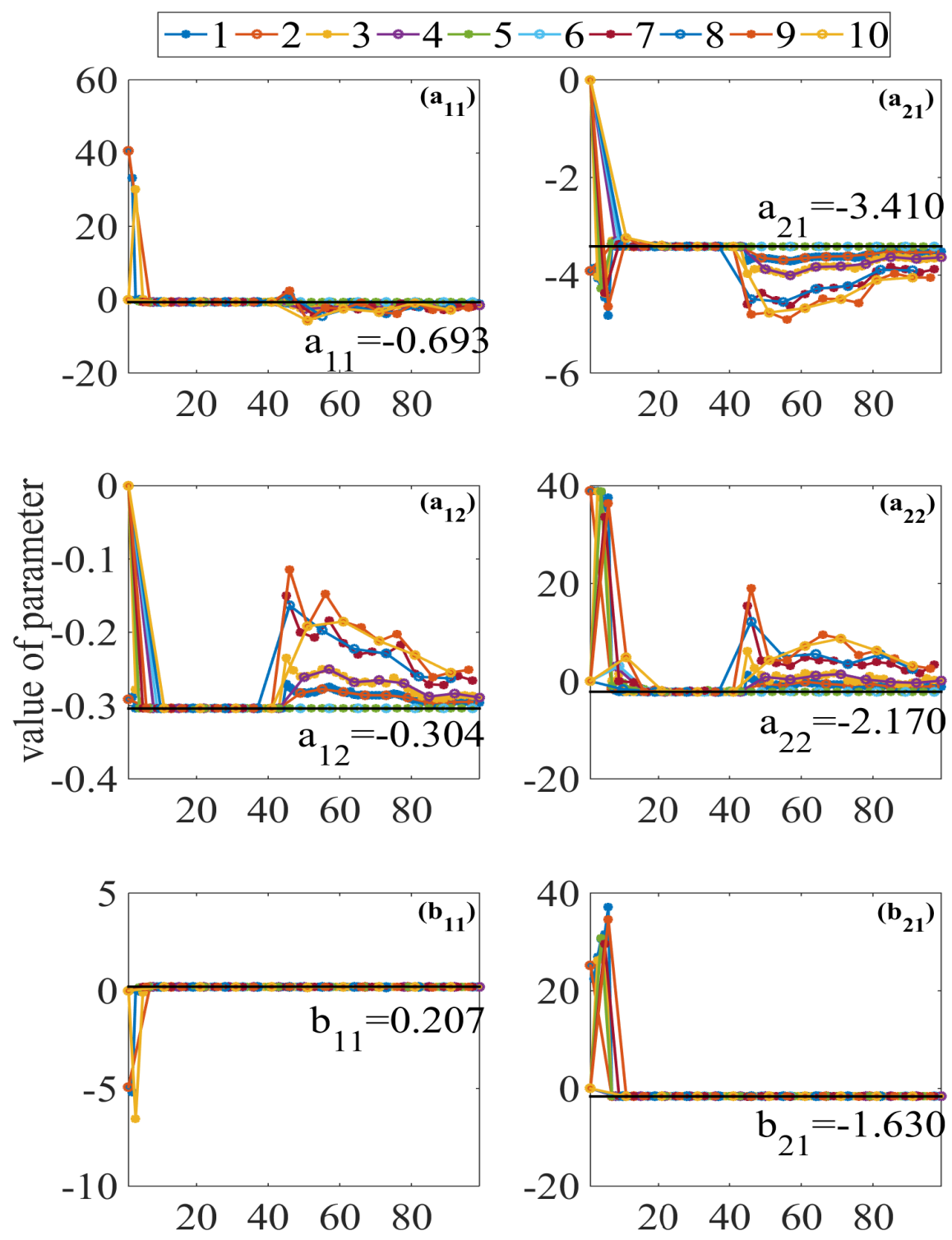

number of samples $(\times 10)$ number of samples $(\times 10)$

Figure 7.3.2: Convergence of LS-SVR in the condition of varying number and interval of samples.

using LSSVR-RLS are illustrated in Figure 7.3.3 where the results from RLS are also presented as a comparison. Table 7.3.2 lists the estimated values and true values of the parameters. Obviously, the fluctuation of convergence of RLS is more intense than that of LSSVR-RLS. The parameters such as $a_{11}, b_{11}$ and $b_{21}$ estimated by LSSVR-RLS, are as precise as those obtained by RLS in terms 
of the relative error between the estimated value and the true value. Besides, $a_{21}$ and $a_{22}$ identified by LSSVR-RLS are more accurate than that identified by RLS, especially $a_{22}$. Comparatively, the estimated parameters of LSSVR-RLS have higher accuracy than that of RLS due to the initial values of these parameters provided by LS-SVR are close to their true values. Additionally, this benefit also contributes to the better convergence performance of LSSVR-RLS. Consequently, it can be concluded that LS-SVR is beneficial to increase convergence speed and improve the identification results of RLS. Note that even applying the noised data, LS-SVR can estimate parameters with accuracy close to the true values, but the LSSVR-RLS cannot ensure this.

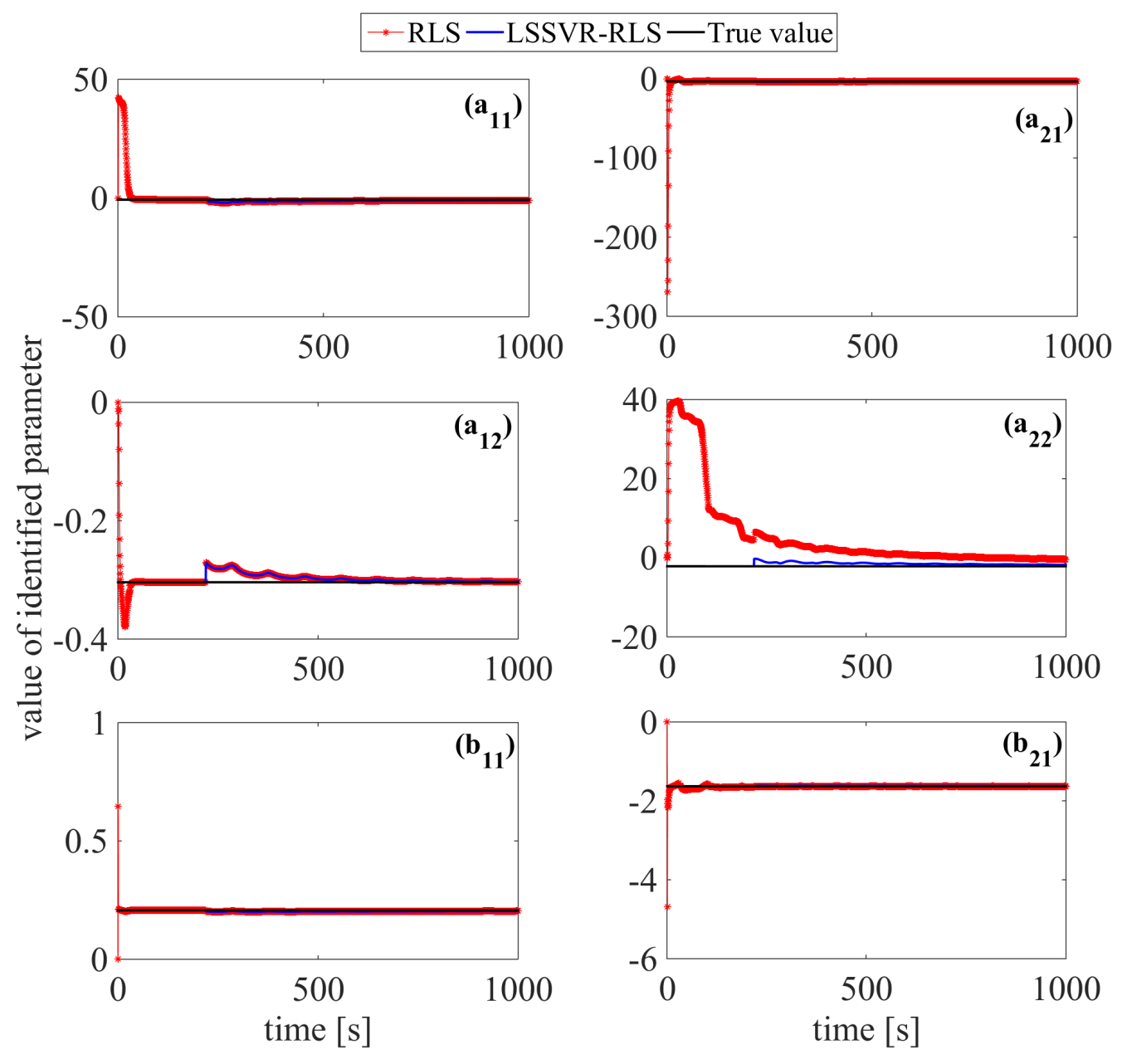

Figure 7.3.3: Identification results of RLS and LSSVR-RLS. 
Table 7.3.2: Identification results of RLS and LSSVR-RLS.

\begin{tabular}{l|llllll}
\hline & $a_{11}$ & $a_{12}$ & $b_{11}$ & $a_{12}$ & $a_{22}$ & $b_{21}$ \\
\hline True value & -0.693 & -0.304 & 0.207 & -3.41 & -2.17 & -1.63 \\
RLS & -0.9891 & -0.3027 & 0.2036 & -3.4579 & -0.4267 & -1.63 \\
Relative error & 0.4273 & -0.0042 & -0.0162 & 0.0140 & -0.8033 & 0 \\
LSSVR-RLS & -0.9895 & -0.3027 & 0.2036 & -3.4538 & -1.7686 & -1.63 \\
Relative error & 0.4278 & -0.0042 & -0.0162 & 0.0129 & -0.1850 & 0 \\
\hline
\end{tabular}

Note: relative error means the subtraction of the estimated value and the true value of a parameter divides the true value.

\subsubsection{VALIDATION}

A $10^{\circ} / 10^{\circ}$ zigzag maneuver is predicted by using the identified steering models, as shown in Figure 7.3.4. In the process of prediction, it can be observed that the identified steering model of RLS is not able to predict zigzag maneuver as expected. On the contrary, a similar trend of $10^{\circ} / 10^{\circ}$ zigzag maneuver is obtained by using the steering model identified by LSSVR-RLS. But the difference between the original maneuver and the predicted one becomes manifest after the third execution of the $10^{\circ}$ rudder angle. The possible aspects of this phenomenon are the relative low identification accuracy of $a_{11}$ and $a_{22}$ and the negative effects of the noises corrupted in the data on identification results. This reason also demonstrates that these two parameters are of much importance to the prediction of the steering model. Much attention should be paid on these two parameters to get more precise values while the identification is conducted, which would improve the generalization performance of the identified model even though the current identified steering model has illustrated it.

\subsection{Case 3: Parameter Estimation of the Simplified Ship Dynamic Model USING ABC-LSSVR}

The merits of LS-SVR have been demonstrated in the previous two case studies. To improve the performance of the LS-SVR based identification method, ABC is used to optimize its regularization parameter. Applying the optimized LS-SVR to identify the simplified ship dynamic model is investigated in this case study, which is also to highlight the contribution of this thesis to developing the optimized identification method based modeling of ship dynamics for different 

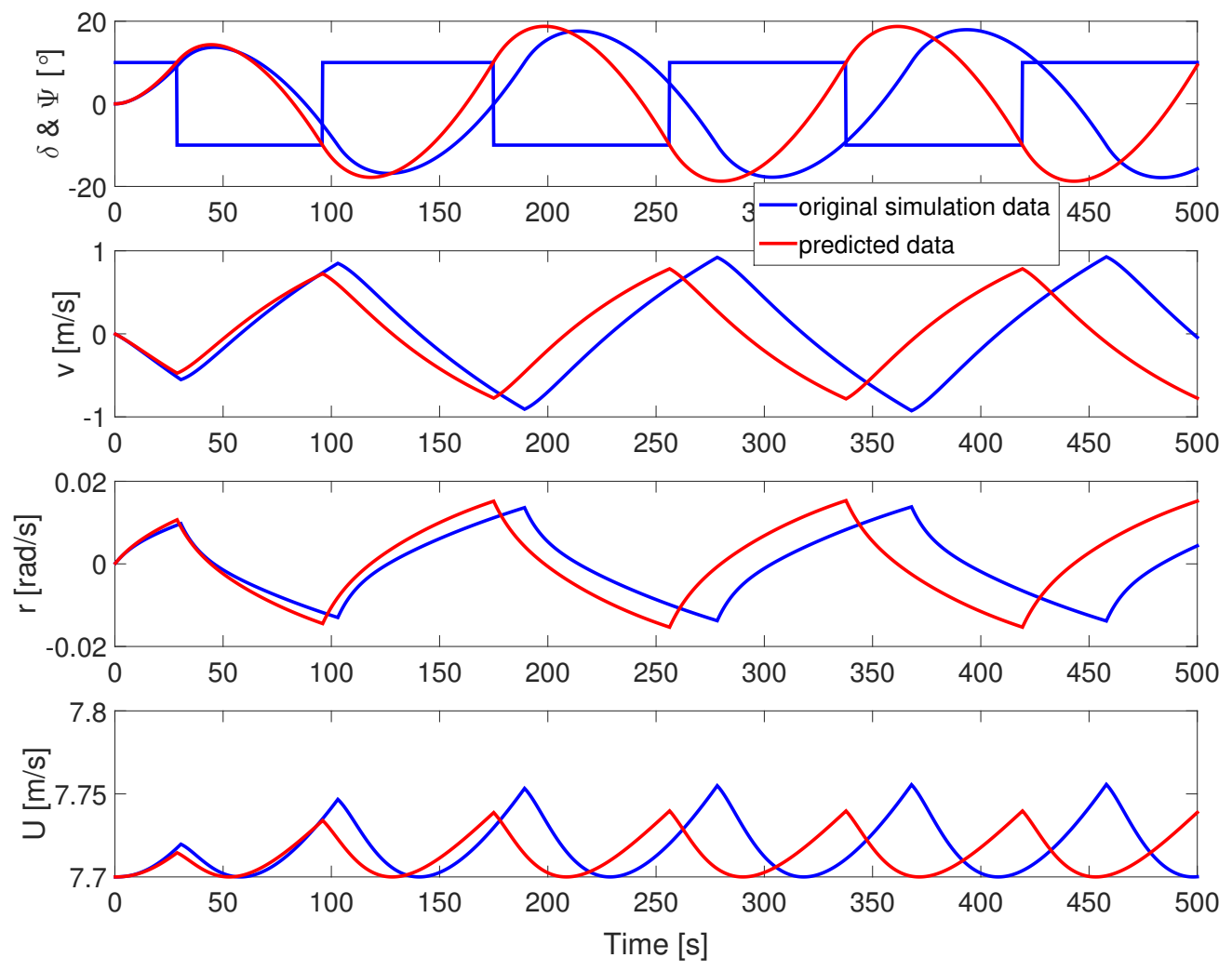

Figure 7.3.4: Predictions of the $10^{\circ} / 10^{\circ}$ zigzag maneuver using the model identified by LSSVR-RLS. The top sub-figure is the commanded rudder angle and the heading angle, the second sub-figure is the sway velocity, the third one is the yaw rate, the bottom sub-figure is the resultant speed.

types of ships.

\subsubsection{Data Processing}

Under a benign environment without external disturbances and measurement noises, the numerical simulation data are the clean data with similar features as the experimental data. The selected model of a large container ship has been well proven by (Fossen 2011 ) and (Son and Nomoto 1982). The corresponding maneuvering simulation data are controllable and used as the alternative of real experimental maneuver data. The numerical simulation is beyond many financial and technical possibilities to do a similar simulation for potential field tests. Good examples refer to (Rajesh and Bhattacharyya 2008), (Jian, Jiayuan, Feng, Jianchuan, Zaojian, Hao, Tao, and Luchun 2015), and (Hou and Zou 2016). 
For parameter estimation, the data used for learning and validation are produced by a 4 DOF dynamic model of the container ship (detailed model is expressed in Appendix D) with predefined parameters extracted from the study in (Son and Nomoto 1982). Four groups of maneuvers including two straight lines with varying propeller shaft speed for estimating the speed model and $10^{\circ} / 10^{\circ}, 20^{\circ} / 20^{\circ}$ zigzag tests for the steering model identification are carried out, respectively. The details are introduced in Table 7.4.1. The data used for regularization parameter optimization of LS-SVR and parameters estimation of the simplified dynamic model are presented in Figure 7.4.1.

Table 7.4.1: The scheme of ship maneuvers.

\begin{tabular}{|c|c|c|c|}
\hline Maneuver & $\begin{array}{l}\text { Commanded } \\
\text { rudder } \\
\text { angle }\left(^{\circ}\right)\end{array}$ & $\begin{array}{l}\text { Propeller shaft } \\
\operatorname{speed}(r p m)\end{array}$ & Purpose \\
\hline Straight line & o & Vary in $[120,160]$ & Identify the surge model \\
\hline Straight line & o & Vary in $[100,160]$ & Verify the identified surge model \\
\hline $10^{\circ} / 10^{\circ}$ zigzag & $\pm_{10}$ & 80 & Identify the steering model \\
\hline $20^{\circ} / 20^{\circ}$ zigzag & \pm 20 & 80 & Verify the identified steering model \\
\hline
\end{tabular}

Note that these maneuvers have the same initial conditions as follows: $U_{\mathrm{o}}=u_{\mathrm{o}}=8 \mathrm{~m} / \mathrm{s}, v_{\mathrm{o}}=\mathrm{o}$, $r_{\mathrm{o}}=\mathrm{o}, \delta_{\mathrm{o}}=\mathrm{o}, \psi_{\mathrm{o}}=\mathrm{o}, n_{\mathrm{o}}=8 \mathrm{orpm}$, the sample time is $900 \mathrm{~s}$, and the interval is $0.5 \mathrm{~s}$.

\subsubsection{IDENTIFIEd Models Using ABC-LSSVR}

Based on the flow of the proposed ABC-LSSVR identification method, the practical procedure for tuning the regularization parameter in LS-SVR by ABC can be concretely depicted as follows: (1) properties of $\mathrm{ABC}$ are set to be: $N P=20, S=20, D=1, x_{j}^{\min }=10^{-2}$, Limit $=20, T=30$; (2) initial positions of the food sources are deployed according to (5.12); (3) employed bees start searching new food sources through $(5.15)$. The selected data including 1800 samples extracted from the first straight line maneuver of the container ship and 1800 samples of the $10^{\circ} 10^{\circ} \mathrm{zigzag}$ maneuver are respectively applied to (5.14) to compute the fitness value via (5.13) for every food source; (4) the best food source is determined by the greedy selection mechanism and replaces the previously stored one. The value of the position of the best food source is the optimal regularization parameter; ( 5 ) onlooker bees take food information from employed bees. Equation (5.16) is used to choose food sources for every onlooker bee. The food sources of onlooker bees are updated via $(5.13)$ and $(5.14) ;(6)$ the employed bee will be to be a scout bee if 


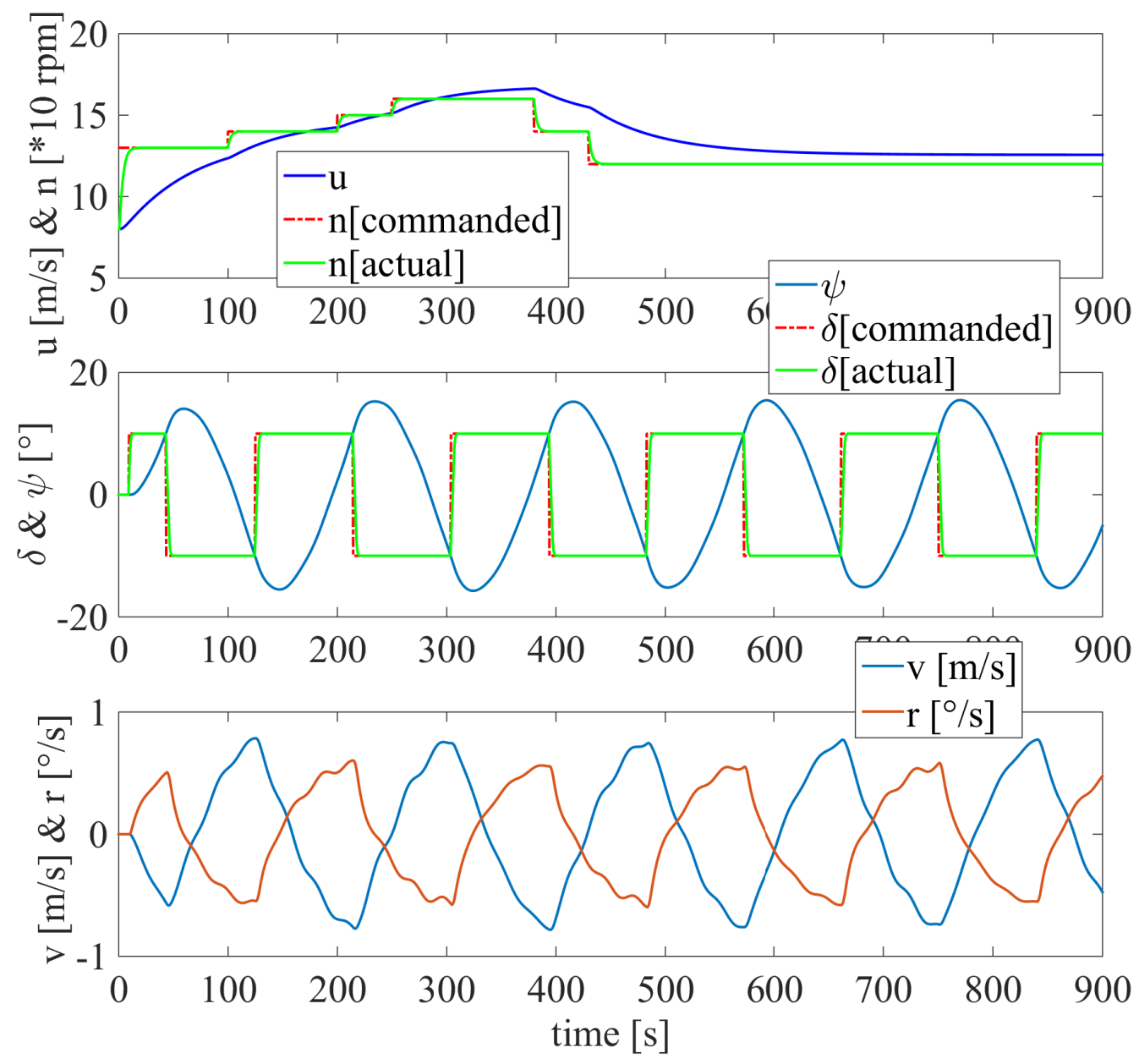

Figure 7.4.1: Simulated data for the optimization and the identification of simplified dynamic models. The top sub-figure presents the surge speed and commanded/actual propeller shaft speed of the first straight line maneuver as described in Table 7.4.1, the middle subfigure donates the heading and commanded/actual rudder angles of the $10^{\circ} / 10^{\circ}$ zigzag maneuver, the bottom sub-figure is the sway speed and yaw rate of the $10^{\circ} / 10^{\circ}$ zigzag maneuver.

two conditions occur. One condition is that the updated fitness value of the food source of the onlooker bee is higher than that of the employed bee. Another condition is that the food fitness of the employed bee is not improved; (7) the position of the food source with the highest fitness value is defined as the optimal regularization parameter when the iteration number is accumulated to 30 .

The best regularization parameters optimized by $\mathrm{ABC}$ are recorded in Table 7.4.2. The ABC-LSSVR regression results are shown in Figure 7.4.2, in which the approximations of 
LS-SVR agree well with the simulations, and the mean squared errors between prediction and simulation results (MSE) of $u\left(4.0346 \times 10^{-6}\right)$, MSE of $v\left(3.5005 \times 10^{-6}\right)$, and MSE of $r\left(4.3924 \times 10^{-6}\right)$ are small enough to be ignored. The optimized LS-SVR is subsequently used to identify the simplified ship dynamic model. The identification results, namely the parameters in $\mathbf{A}, \mathbf{B}$, and $\mathbf{C}$, are given in Table 7.4.3.

Table 7.4.2: The best regularization parameter and MSE.

\begin{tabular}{clll}
\hline \multirow{2}{*}{ Best regularization value } & SM & SS & SY \\
\cline { 2 - 4 } & $4.3313 \times 10^{7}$ & $8.9486 \times 10^{9}$ & 19.9067 \\
\hline \multirow{2}{*}{ MSE } & $\mathrm{SM}\left(\mathrm{m}^{2} / \mathrm{s}^{2}\right)$ & $\mathrm{SS}\left(\mathrm{m}^{2} / \mathrm{s}^{2}\right)$ & $\mathrm{SY}\left(\mathrm{o}^{2} / \mathrm{s}^{2}\right)$ \\
\cline { 2 - 4 } & $4.0346 \times 10^{-6}$ & $3.5005 \times 10^{-6}$ & $4.3924 \times 10^{-6}$ \\
\hline
\end{tabular}

Note that (SM) surge model; (SS) steering model (sway motion); (SY) steering model (yaw motion).

Table 7.4.3: Parameters estimated by ABC-LSSVR.

\begin{tabular}{cccccccc}
\hline \multirow{2}{*}{$A$} & $a_{1}$ & $a_{2}$ & $a_{3}$ & & & & \\
\cline { 2 - 7 } & 9942 & -1.603 & 85.962 & & & & \\
\hline \multirow{2}{*}{$B$} & $b_{1}$ & $b_{2}$ & $b_{3}$ & $b_{4}$ & $b_{5}$ & $b_{6}$ & $b_{7}$ \\
\cline { 2 - 7 } & 0.9743 & 0.0043 & 1.5306 & -1.6533 & 3.5656 & -140.312 & -0.0278 \\
\hline \multirow{2}{*}{$C$} & $c_{1}$ & $c_{2}$ & $c_{3}$ & $c_{4}$ & $c_{5}$ & $c_{6}$ & $c_{7}$ \\
\cline { 2 - 7 } & -0.0092 & 0.0002 & -0.0010 & 0.0223 & 0.0024 & 0.0001 & 0.0153 \\
\hline
\end{tabular}

\subsubsection{VALIDATION}

The second straight line and $20^{\circ} / 20^{\circ}$ zigzag maneuvers are predicted by using the identified surge and steering models, respectively. The original simulation data of these maneuvers are generated by the 4 DOF model of the container ship. As presented in Figure 7.4.3, the tendency of the predicted data is very similar to that of the original simulation. However, the obvious deviations between the original data and predicted data are observed on the moment when the rudder command is executed. The predicted surge speed is a little higher than the simulated surge speed, but it can still illustrate that ABC-LSSVR performs good generalization because the small errors 

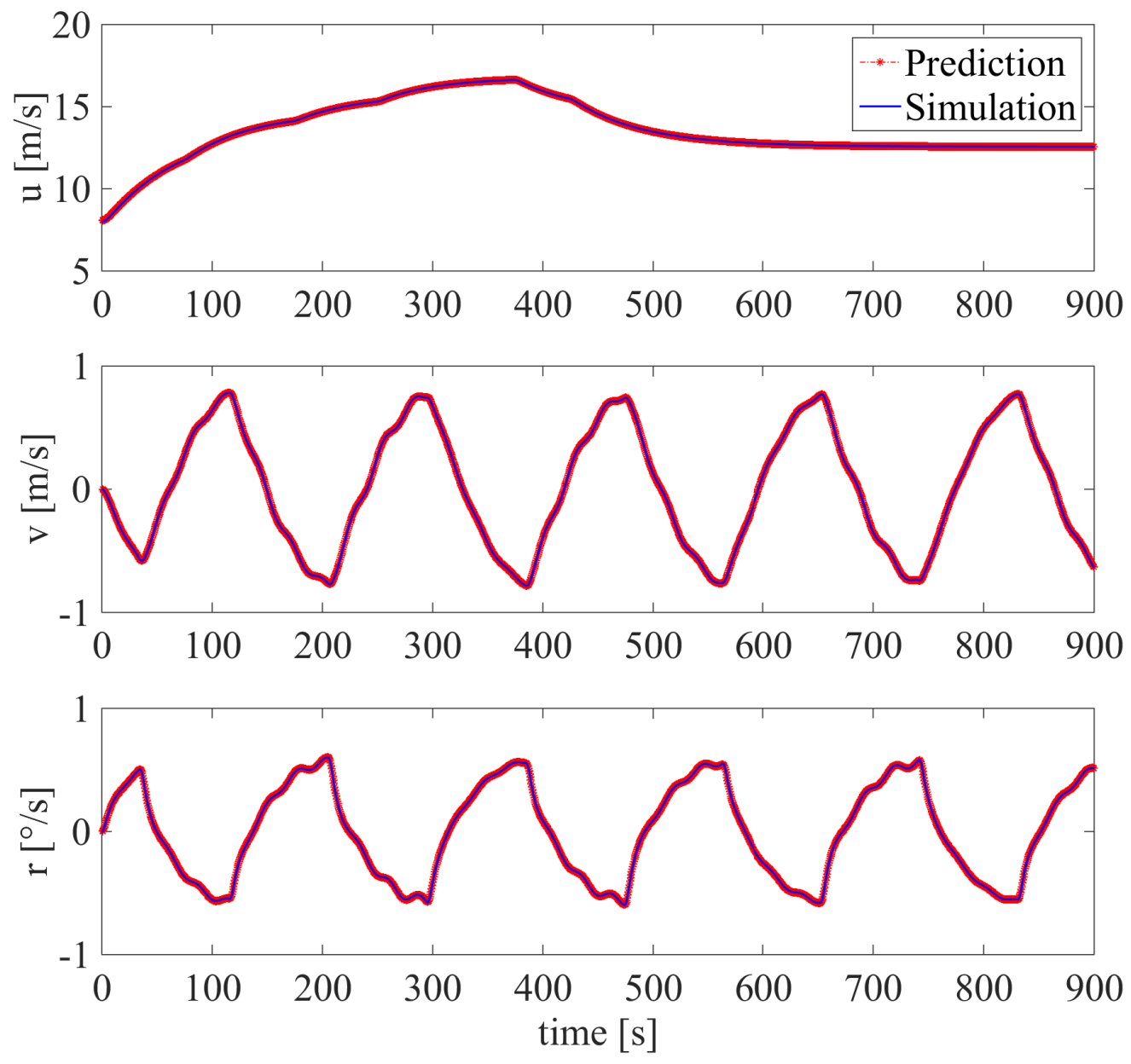

Figure 7.4.2: Regression of the ABC-LSSVR. The upper sub-figure is the surge speed of the first straight line maneuver, the middle one is the sway speed of the $10^{\circ} / 10^{\circ}$ zigzag maneuver, the lower one is the yaw rate of the $10^{\circ} / 10^{\circ}$ zigzag maneuver.

in surge speed predictions are not significant for practical aspects or to affect the applicability of the ABC-LSSVR method. The aspects for arising the errors in predictions is that the underlying physical characteristics of this container ship, the ignorance of the coupling effects among the surge, steering and roll motions, and the effects of parameter drift. These aspects are highly related to the modeling of ship dynamics. So, it is worth to be noticed that the errors existing in predictions make few influences on the applicability and effectiveness of the proposed ABC-LSSVR method to identify the simplified ship dynamic model. To improve the predictions, modifying the simplified dynamic model is necessitated. 

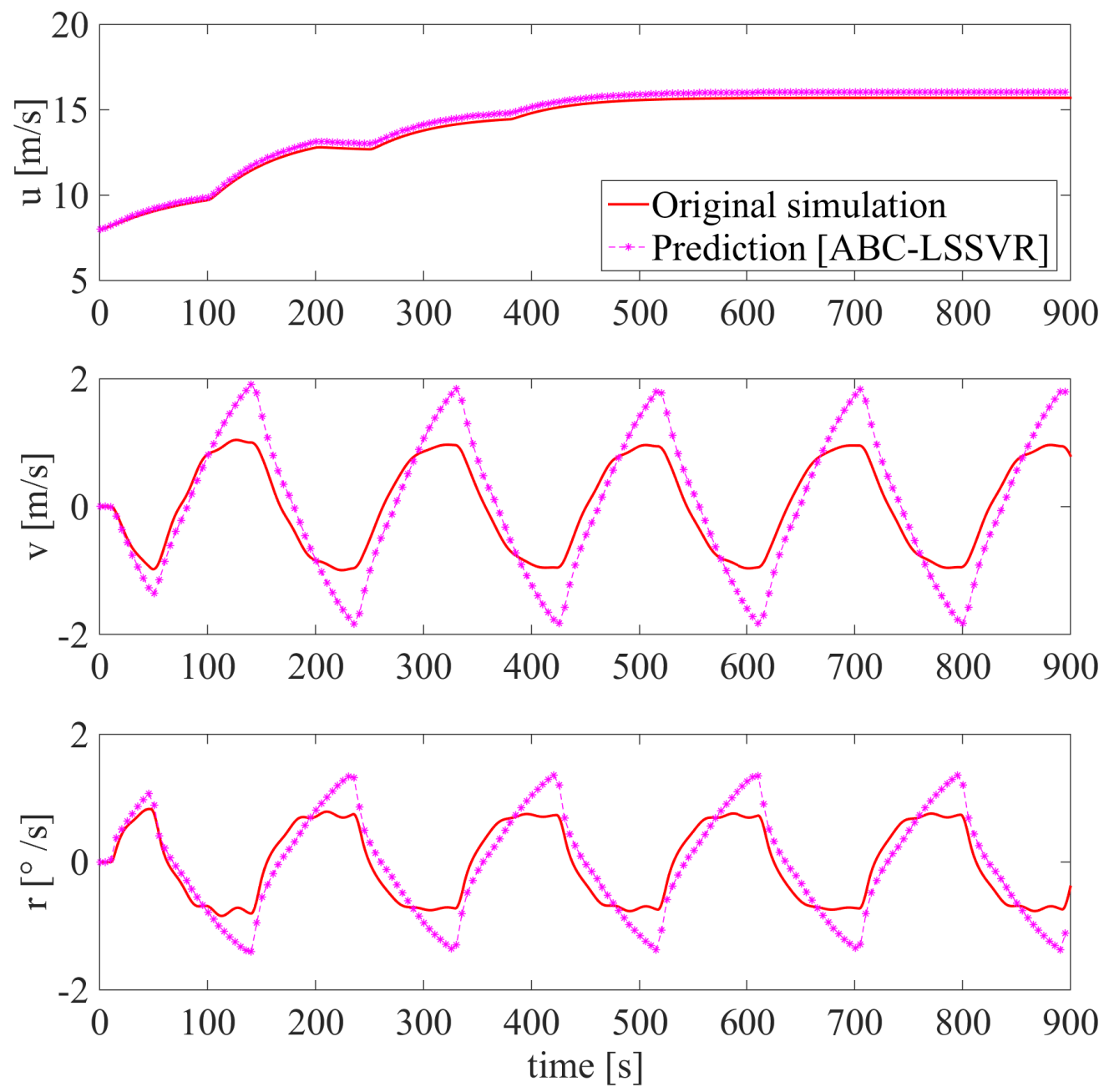

Figure 7.4.3: Predictions of ship dynamics using models identified by the ABC-LSSVR. The upper sub-figure is the surge velocity of the second straight line maneuver, the middle one is the sway speed of the $20^{\circ} / 20^{\circ}$ zigzag maneuver, the bottom one is the yaw rate of the $20^{\circ} / 20^{\circ}$ zigzag maneuver.

\subsubsection{Comparison of Three Optimized LS-SVRs}

In order to thoroughly indicate the effectiveness of $A B C$ tuning LS-SVR, the comparisons of LS-SVR optimized by CV (CV-LSSVR), and by PSO (PSO-LSSVR) have been carried out. For quantitatively analyzing and comparing the performance of these three parameter identification methods, three indexes including the computational time, the best regularization parameter and 
Table 7.4.4: Comparison of the computational time, the best regularization parameter, and MSE.

\begin{tabular}{|c|c|c|c|}
\hline \multirow{2}{*}{ Method } & \multicolumn{3}{|c|}{ Computational time(s) } \\
\hline & SM & SS & SY \\
\hline $\mathrm{CV}$ & 0.2318 & 0.2450 & 0.2729 \\
\hline PSO & 0.3181 & 0.2659 & 0.2553 \\
\hline $\mathrm{ABC}$ & 0.7143 & 0.2700 & 0.3285 \\
\hline \multirow{2}{*}{ Method } & \multicolumn{3}{|c|}{ Best regularization parameter } \\
\hline & $\operatorname{SM}\left(\times 10^{7}\right)$ & SS $\left(\times 10^{9}\right)$ & SY \\
\hline $\mathrm{CV}$ & 0.2318 & 0.8968 & 14.7966 \\
\hline PSO & 1.7492 & 6.0000 & 19.7130 \\
\hline $\mathrm{ABC}$ & 4.3313 & 19.9067 & 0.1289 \\
\hline \multirow{2}{*}{ Method } & \multicolumn{3}{|c|}{$\operatorname{MSE}\left[20^{\circ} / 20^{\circ}\right]$} \\
\hline & $\operatorname{SM}\left(m^{2} / s^{2}\right)$ & $\mathrm{SS}\left(m^{2} / s^{2}\right)$ & $\mathrm{SY}\left(\mathrm{O}^{2} / s^{2}\right)$ \\
\hline $\mathrm{CV}$ & 0.5296 & 1.6221 & 1.0096 \\
\hline PSO & 0.4868 & 0.2340 & 0.1903 \\
\hline $\mathrm{ABC}$ & 0.1289 & 0.3380 & 0.1196 \\
\hline
\end{tabular}

Note: (SM) surge model; (SS) steering model (sway motion); (SY) steering model (yaw motion); (CV) CV-LSSVR; (PSO) PSO-LSSVR; (ABC) ABC-LSSVR.

MSE are recorded in Table 7.4.4. The results of these three methods in identifying the simplified ship dynamic model can be found in Table 7.4.5, Table 7.4.6, and Table 7.4.7. The prediction results of the second straight line and $20^{\circ} / 20^{\circ}$ zigzag maneuvers are shown in Figure 7.4.4. As seen, ABC-LSSVR, PSO-LSSVR, and CV-LSSVR can identify the simplified ship dynamic model. However, compared with PSO-LSSVR and ABC-LSSVR, CV-LSSVR presents a relatively poor performance on identifying high precise parameters of the model on account of the three indexes. A similar performance on identifying the steering model of ABC-LSSVR and PSO-LSSVR can be observed, but the errors of surge speed prediction using the surge model identified by ABC-LSSVR are smaller than that of the model identified by PSO-LSSVR. In some degree, this difference between PSO-LSSVR and ABC-LSSVR reveals the outperformance of ABC-LSSVR. From the differences existing in each index, it can be concluded that the performance of ABC-LSSVR is slightly superior to the other two methods from the scientific 
Table 7.4.5: Parameters of the surge model estimated by CV-LSSVR, PSO-LSSVR, and ABC-LSSVR, respectively.

\begin{tabular}{llll}
\hline $\mathbf{A}\left(\times 10^{-5}\right)$ & $a_{1}$ & $a_{2}$ & $a_{3}$ \\
\hline CV-LSSVR & 99420 & -1.8293 & 85.926 \\
PSO-LSSVR & 99340 & -0.9270 & 84.218 \\
ABC-LSSVR & 99420 & -1.8484 & 85.739 \\
\hline
\end{tabular}

Table 7.4.6: Parameters of the sway model estimated by CV-LSSVR, PSO-LSSVR, and ABC-LSSVR, respectively.

\begin{tabular}{llllllll}
\hline B & $b_{1}$ & $b_{2}$ & $b_{3}$ & $b_{4}$ & $b_{5}$ & $b_{6}$ & $b_{7}$ \\
\hline CV-LSSVR & 0.9744 & 0.0045 & 1.4094 & -1.6364 & 3.5224 & -145.7 & -0.0278 \\
PSO-LSSVR & 0.9743 & 0.0043 & 1.5306 & -1.6533 & 3.5656 & -140.3 & -0.0278 \\
ABC-LSSVR & 0.9743 & 0.0045 & 1.5075 & -1.6488 & 3.573 & -142.2 & -0.0278 \\
\hline
\end{tabular}

Table 7.4.7: Parameters of the yaw model estimated by CV-LSSVR, PSO-LSSVR, and ABCLSSVR, respectively.

\begin{tabular}{llllllll}
\hline $\mathbf{C}$ & $c_{1}$ & $c_{2}$ & $c_{3}$ & $c_{4}$ & $c_{5}$ & $c_{6}$ & $c_{7}$ \\
\hline CV-LSSVR & -0.0104 & 0.0002 & -0.0011 & 0.0235 & 0.0024 & 0.0001 & 0.0153 \\
PSO-LSSVR & -0.0110 & 0.0002 & -0.0035 & 0.0230 & 0.0025 & 0.0001 & 0.0152 \\
ABC-LSSVR & -0.0092 & 0.0002 & -0.0010 & 0.0223 & 0.0024 & 0.0001 & 0.0153 \\
\hline
\end{tabular}

research point of view.

From the numerical simulation study, the different characteristics of these optimization methods are observed and concluded. CV as a trial and error method is a little time-consuming to find a suitable solution for the current problem. The solution is not so optimal as the optimization results of PSO and ABC. PSO can find the global optimum for LS-SVR, but sometimes it fails to provide globally optimal solutions instead of locally optimal ones. Comparatively, ABC shows relatively strong global optimization ability during the selection of regularization parameter for LS-SVR. In summary, CV could be an option for selecting parameters of LS-SVR based on reliable experiences, which is suitable for offline parameter identification. $A B C$ would be better than PSO to optimize LS-SVR, and the combination of ABC and modified LS-SVR could be a potential application for online parameter identification in terms of computational time and finite samples. 

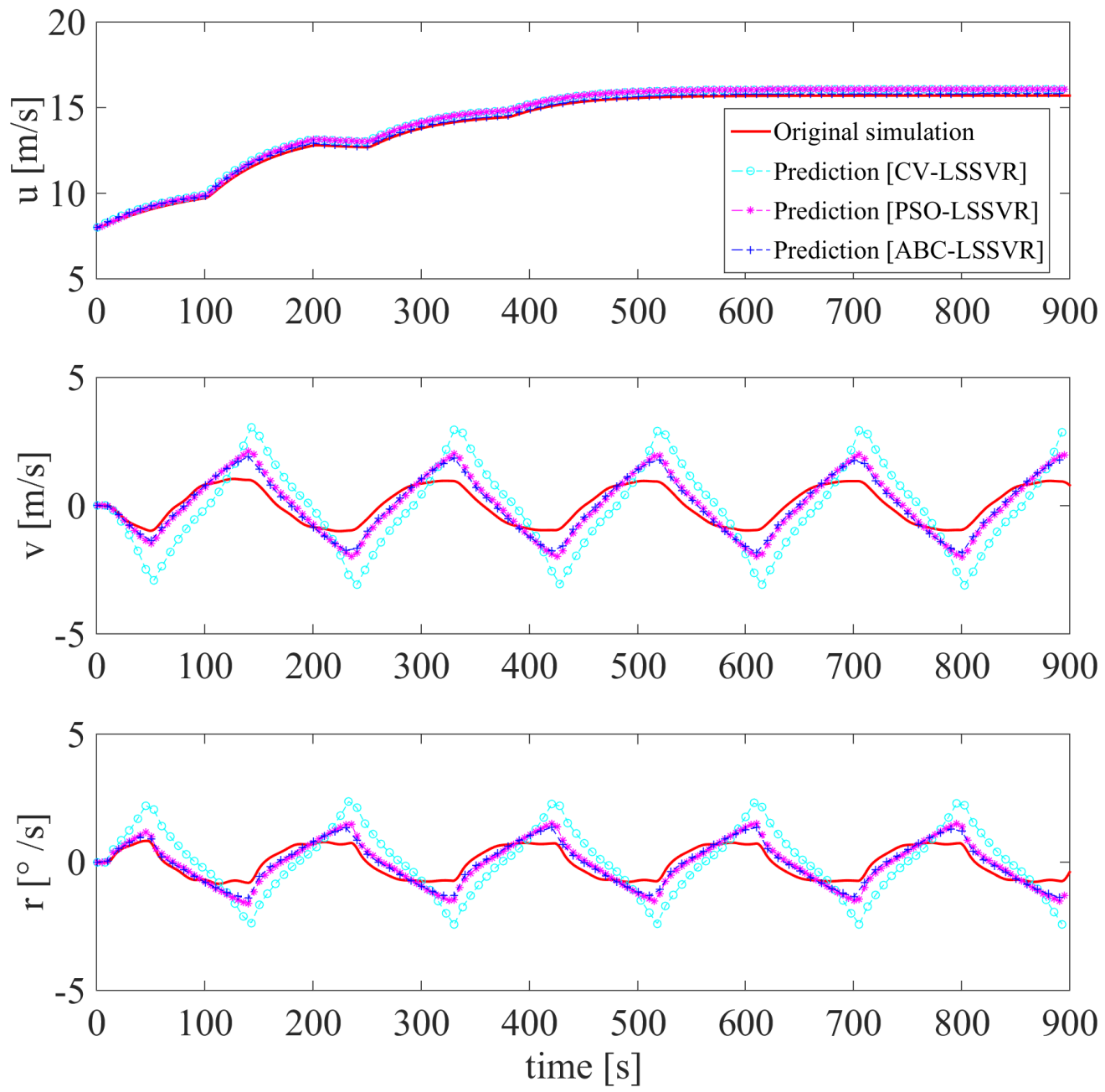

Figure 7.4.4: Comparison of the three ship dynamic models identified by CV-LSSVR, PSOLSSVR, and ABC-LSSVR, respectively. The top sub-figure is the predictions of surge velocity, the middle one is the predictions of sway velocity of the $20^{\circ} / 20^{\circ}$ zigzag maneuver, the bottom sub-figure is the predicted yaw rate of the $20^{\circ} / 20^{\circ}$ zigzag maneuver.

\subsubsection{COMparison of Steering Models}

Nomoto models are widely used to describe a ship's heading reaction to the commanded rudder order due to its simplicity and easy-understanding. The first order Nomoto models are compared with the proposed nonlinear steering model to present the performance of the nonlinear model. The data used previously for the identification of the nonlinear steering model are also utilized by 
ABC-LSSVR with the same property settings to identify the first order Nomoto models and validate the identification results. The predictions of $20^{\circ} / 20^{\circ}$ zigzag maneuver generated by these three identified steering models are shown in Figure 7.4.5, and the identification results of the steering quality indices and the related MSE are given in Table 7.4.8. It can be observed that the performances of the two Nomoto models are almost the same on account of MSE and the estimated maneuvering indices. However, the nonlinear steering model underperforms these Nomoto models in terms of MSE. From the practical engineering point of view, this difference makes few influences on deciding which model is to describe yaw motion, but the first order linear/nonlinear Nomoto model is suggested as the better choice for control design because of its simple construction. This difference is probably due to the parameter drift, which is a common issue in the statistical regression analysis, involved in the ABC-LSSVR based identification. In the estimation of ship dynamic models, two types of parameter drift including the drift of linear hydrodynamic coefficients and the drift of the nonlinear hydrodynamic coefficients affect, which can be explained respectively through the analysis of dynamic cancellation and statistical regression analysis. Measures used effectively to mitigate the influence of parameter drift on ABC-LSSVR based identification of the nonlinear steering model are usually categorized into three core kinds, i.e., the simplification of the model, the difference method in previously dealing with data, and additional signals added to data. Therefore, the special considerations concerning the further simplification of the nonlinear steering model are conducted in Section 7.4.6 to improve the accuracy of the simplified model. This consideration contributes to the objectives of this thesis.

Table 7.4.8: Identification results of Nomoto models.

\begin{tabular}{ccccc}
\hline Model & $K(1 / s)$ & $T(s)$ & $n_{3}\left(\times 10^{-11}\right)$ & $M S E\left[S Y\left(0^{2} / s^{2}\right)\right]$ \\
\hline NSM & & & & 0.1196 \\
LNM & 0.031 & 30.2487 & & 0.0548 \\
NNM & 0.031 & 30.2487 & 7.0853 & 0.0548 \\
\hline
\end{tabular}

Note: (NSM) nonlinear steering model; (LNM) the first order linear Nomoto model; (NNM) the first order nonlinear Nomoto model. 


\section{CHAPTER 7. EVALUATION}

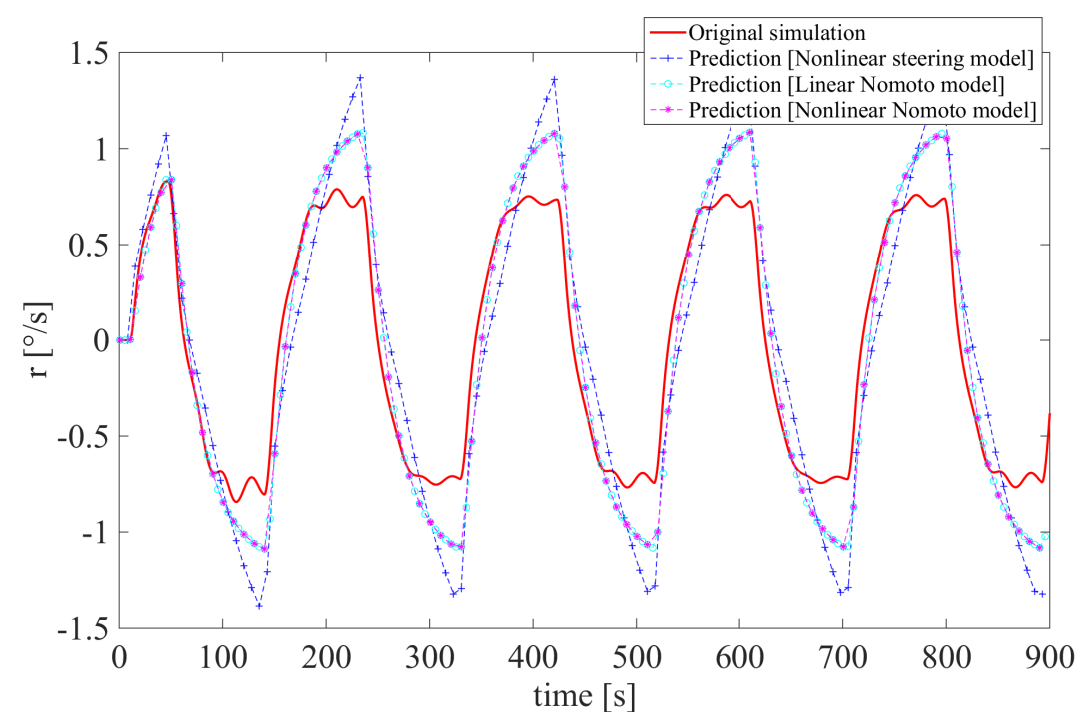

Figure 7.4.5: Predictions of $20^{\circ} / 20^{\circ}$ zigzag maneuver using different identified steering models.

\subsubsection{Special Considerations: Further Simplification of the Nonlinear Steering MODEL}

To intuitively present the further simplification process, the simplified nonlinear steering model is rewrote into the form $(7 \cdot 1)$.

$$
\begin{aligned}
& \dot{v}=b_{v} v+b_{|v| v}|v| v+b_{|v| v}|r| v+b_{r} r+b_{|v| r}|v| r+b_{|r| r}|r| r+b_{\delta} \delta \\
& \dot{r}=c_{v} v+c_{|v| v}|v| v+c_{|v| v}|r| v+c_{r} r+c_{|v| r}|v| r+c_{|r| r}|r| r+c_{\delta} \delta
\end{aligned}
$$

where

$$
\begin{aligned}
b_{v}= & \frac{\left(I_{z}-N_{\dot{r}}\right) Y_{v}+\left(\left(m-X_{\dot{u}}\right) u_{\mathrm{o}}+N_{v}\right)\left(Y_{\dot{r}}-m x_{g}\right)}{\nabla} \\
b_{r}= & \frac{\left(I_{z}-N_{\dot{r}}\right) Y_{|v| v}-\left(m x_{g}-Y_{\dot{r}}\right) N_{|v| v}}{\nabla} \\
b_{|v| v} & =\frac{\left(I_{z}-N_{\dot{r}}\right) Y_{|r| v}-\left(m x_{g}-Y_{\dot{r}}\right) N_{|r| v}}{\nabla} \\
b_{r v} & =\frac{\left(I_{z}-N_{\dot{r}}\right)\left(Y_{r}-\left(m-X_{\dot{u}}\right) u_{\mathrm{o}}\right)-\left(m x_{g}-Y_{\dot{r}}\right) N_{r}}{\nabla}
\end{aligned}
$$




$$
\begin{aligned}
b_{|v| r}= & \frac{\left(I_{z}-N_{\dot{r}}\right) Y_{|v| r}-\left(m x_{g}-Y_{\dot{r}}\right) N_{|v| r}}{\nabla} \\
b_{|r| r}= & \frac{\left(I_{z}-N_{\dot{r}}\right) Y_{|r| r}-\left(m x_{g}-Y_{\dot{r}}\right) N_{|r| r}}{\nabla} \\
b_{\delta} & =\frac{\left(I_{z}-N_{\dot{r}}\right) Y_{\delta}-\left(m x_{g}-Y_{\dot{r}}\right) N_{\delta}}{\nabla} \\
c_{v}= & \frac{\left(N_{\dot{v}}-m x_{g}\right) Y_{v}+\left(m-Y_{\dot{v}}\right)\left(\left(m-X_{\dot{u}}\right) u_{\mathrm{o}}+N_{v}\right)}{\nabla} \\
c_{r}= & \frac{-\left(N_{\dot{v}}-m x_{g}\right) Y_{|v| v}-\left(m-Y_{\dot{v}}\right) N_{|v| v}}{\nabla} \\
\mathcal{c}_{|v| v}= & \frac{\left(N_{\dot{v}}-m x_{g}\right) Y_{|v| v}+\left(m-Y_{\dot{v}}\right) N_{|v| v}}{\nabla} \\
\mathcal{c}_{|r| v}= & \frac{\left(m-Y_{\dot{v}}\right) N_{r}-\left(N_{\dot{v}}-m x_{g}\right)\left(\left(m-X_{\dot{u}}\right) u_{\mathrm{o}}-Y_{r}\right)}{\nabla} \\
\mathcal{c}_{|v| r}= & \frac{\left(N_{\dot{v}}-m x_{g}\right) Y_{|v| r}+\left(m-Y_{\dot{v}}\right) N_{|v| r}}{\nabla} \\
\mathcal{c}_{|r| r}= & \frac{\left(N_{\dot{v}}-m x_{g}\right) Y_{|r| r}+\left(m-Y_{\dot{v}}\right) N_{|r| r}}{\nabla} \\
\mathcal{c}_{\delta}= & \frac{\left(N_{\dot{v}}-m x_{g}\right) Y_{\delta}+\left(m-Y_{\dot{v}}\right) N_{\delta}}{\nabla}
\end{aligned}
$$

and $\nabla=\left(m-Y_{\dot{v}}\right)\left(I_{z}-N_{\dot{r}}\right)-\left(m x_{g}-Y_{\dot{r}}\right)\left(m x_{g}-N_{\dot{v}}\right)$.

Several groups of simplification of the nonlinear steering model are carried out by picking terms from the nonlinear steering model and then recombining them together, the corresponding sway and yaw parameters of the reconstructed model estimated by ABC-LSSVR are listed in Table 7.4.9 and Table 7.4.10 where the MSE of the predicted $20^{\circ} / 20^{\circ}$ zigzag maneuver is also included. The identified values of linear terms such as $b_{v}, b_{r}, b_{\delta}, c_{v}, c_{r}$ and $c_{\delta}$ are physically consistent. The MSE of further simplified models in the first and fourth row is not obtained, for which the inconsistent estimated parameters should be responsible. By comparing the MESs, the combination of the further simplified sway model in the sixth row of Table 7.4.9 and yaw model in the same row of Table 7.4.10 is indicated to be the best one describing steering dynamics. Thus, the further simplified steering model with higher accuracy than the nonlinear one is obtained for the container ship, which is expressed as

$$
\begin{aligned}
& \dot{v}=-0.0476 v-3.7649 r+0.9208|v| r-0.064 \delta \\
& \dot{r}=-0.0005 v-0.0691 r-0.0285|v| r+0.0029 \delta
\end{aligned}
$$

The prediction results of $20^{\circ} / 20^{\circ}$ zigzag maneuver with the comparison of the first order Nomoto 
models are depicted in Figure 7.4.6.

Table 7.4.9: Estimated parameters of the sway model.

\begin{tabular}{lllllllll}
\hline Model & $b_{v}$ & $b_{r}$ & $b_{|v| v}$ & $b_{r v}$ & $b_{|v| r}$ & $b_{|r| r}$ & $b_{\delta}$ & $\mathrm{MSE}\left(\mathrm{m}^{2} / s^{2}\right)$ \\
\hline 1 & & & 0.0264 & -10.102 & -0.8184 & -704.84 & -0.0769 & $\mathrm{NaN}$ \\
\hline 2 & -0.0419 & -3.3219 & -0.0280 & & & -105.78 & -0.0542 & 0.0604 \\
\hline 3 & -0.0400 & -3.5520 & -0.0338 & & -1.2368 & & -0.0568 & 0.0495 \\
\hline 4 & & & 0.0264 & -10.101 & -0.8215 & -704.62 & -0.0770 & $\mathrm{NaN}$ \\
\hline 5 & -0.0481 & -3.7079 & & -0.7618 & & & -0.0637 & 0.0472 \\
\hline 6 & -0.0476 & -3.7649 & & & 0.9208 & -0.0637 & 0.0472 \\
\hline 7 & -0.0532 & -3.6720 & & & & -0.0606 & 0.0411 \\
\hline 8 & -0.0025 & & -0.0183 & & & -0.1153 & 0.0413 \\
\hline 9 & & -3.6746 & -0.0184 & & & -0.0610 & 0.0462 \\
\hline 10 & -0.0094 & & & -0.6068 & & -0.1179 & 0.0730 \\
\hline
\end{tabular}

Table 7.4.10: Estimated parameters of the yaw model.

\begin{tabular}{lllllllll}
\hline Model $c_{v}$ & $c_{r}$ & $c_{|v| v}$ & $c_{r v}$ & $c_{|v| r}$ & $c_{|r| r}$ & $c_{\delta}$ & $\operatorname{MSE}\left(0^{2} / s^{2}\right)$ \\
\hline 1 & & & -0.0300 & -0.0026 & 0.0015 & 0.0004 & 0.0300 & $\mathrm{NaN}$ \\
\hline 2 & -0.0007 & -0.0799 & 0.0007 & & & 2.4079 & 0.0026 & 0.0688 \\
\hline 3 & -0.0007 & -0.0738 & 0.0008 & & 0.0198 & & 0.0027 & 0.0646 \\
\hline 4 & & & -0.0016 & 0.1267 & -0.1546 & 17.1446 & 0.0023 & $\mathrm{NaN}$ \\
\hline 5 & -0.0006 & -0.0706 & & 0.0280 & & & 0.0029 & 0.0169 \\
\hline 6 & -0.0005 & -0.0691 & & & -0.0285 & & 0.0029 & 0.0162 \\
\hline 7 & -0.0004 & -0.0720 & & & & & 0.0028 & 0.0684 \\
\hline 8 & & -0.0436 & & & & 0.6847 & 0.0023 & 0.1346 \\
\hline 9 & -0.0004 & & -0.4861 & & & & 0.0028 & 0.0407 \\
\hline 10 & & -0.0335 & & 0.0069 & & & 0.0022 & 0.0447 \\
\hline
\end{tabular}

\subsubsection{Time Complexity Analysis}

To investigate the time complexity of the proposed identification method, a series of numerical simulations in identifying surge and steering models are carried out under the condition of the varying number of training samples with the steps of 10 . The samples used here are the same as the ones adopted previously in this case study section. In the simulation of each group with the 


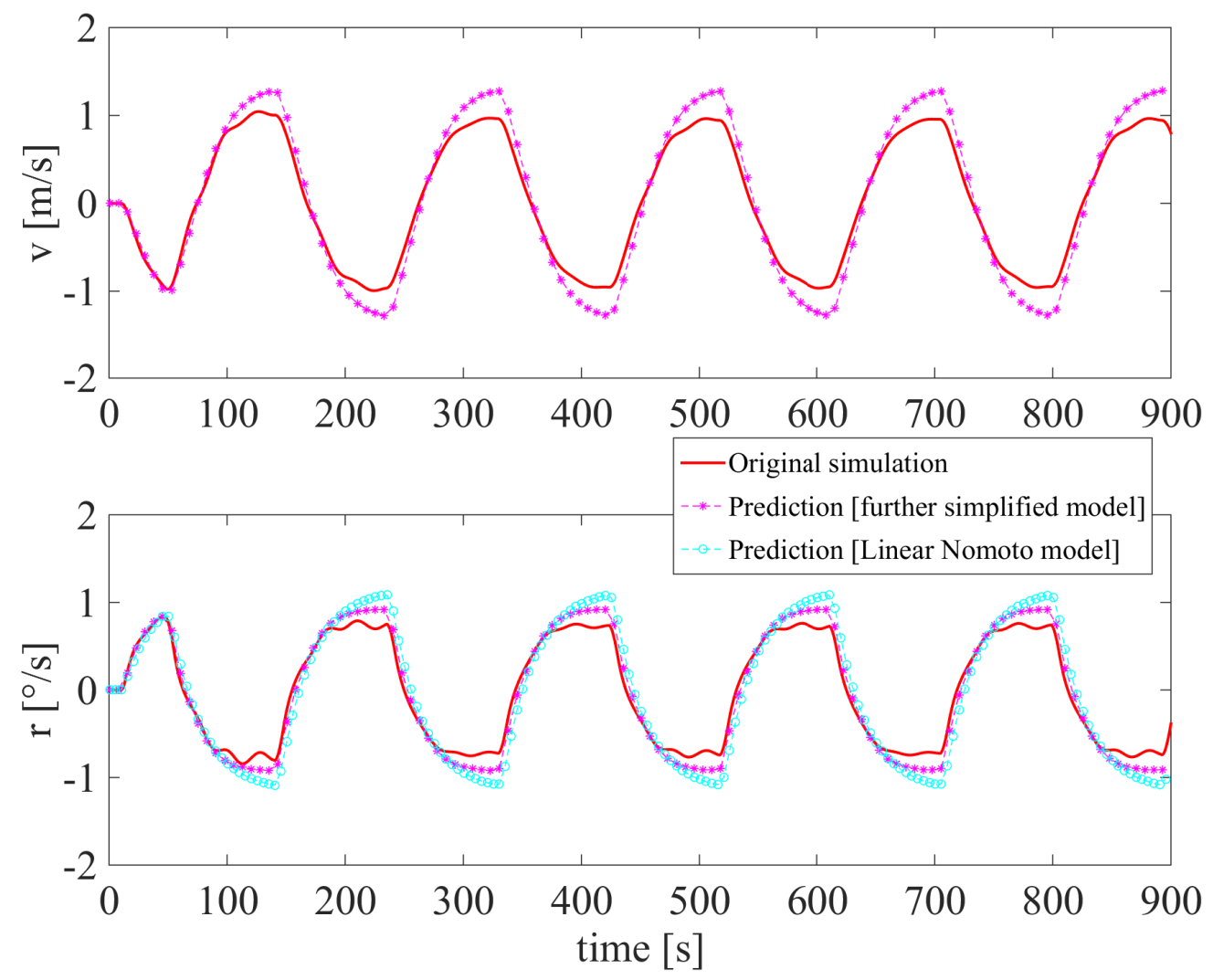

Figure 7.4.6: Predictions of $20^{\circ} / 20^{\circ}$ zigzag maneuver using the further simplified steering model. The top sub-figure is the predictions of sway velocity, the bottom sub-figure is the predicted yaw rate.

use of a specified number of samples, three indexes including the total cost time, MSE of the identified model, and estimated parameters are analyzed. Figures 7-4.7-7.4.8 present the comparison of the identification results of ABC-LSSVR, CV-LSSVR, and PSO-LSSVR on the simplified surge model and further simplified steering model in terms of the consumed time, respectively. From these figures, one can see that the cost time of the three identification methods increases with the increase of the number of samples. This implies that the number of samples has a direct effect on the time complexity of the proposed identification method, which is consistent with the analysis of time complexity in the complexity analysis section. Compared to CV-LSSVR and PSO-LSSVR, ABC-LSSVR takes approximately equivalent time to execute the identification.

The MSE and the estimated parameters of the models identified by the three estimation methods are summarized in Figures 7.4.9-7.4.13. It can be observed that the fluctuation of MSEs 


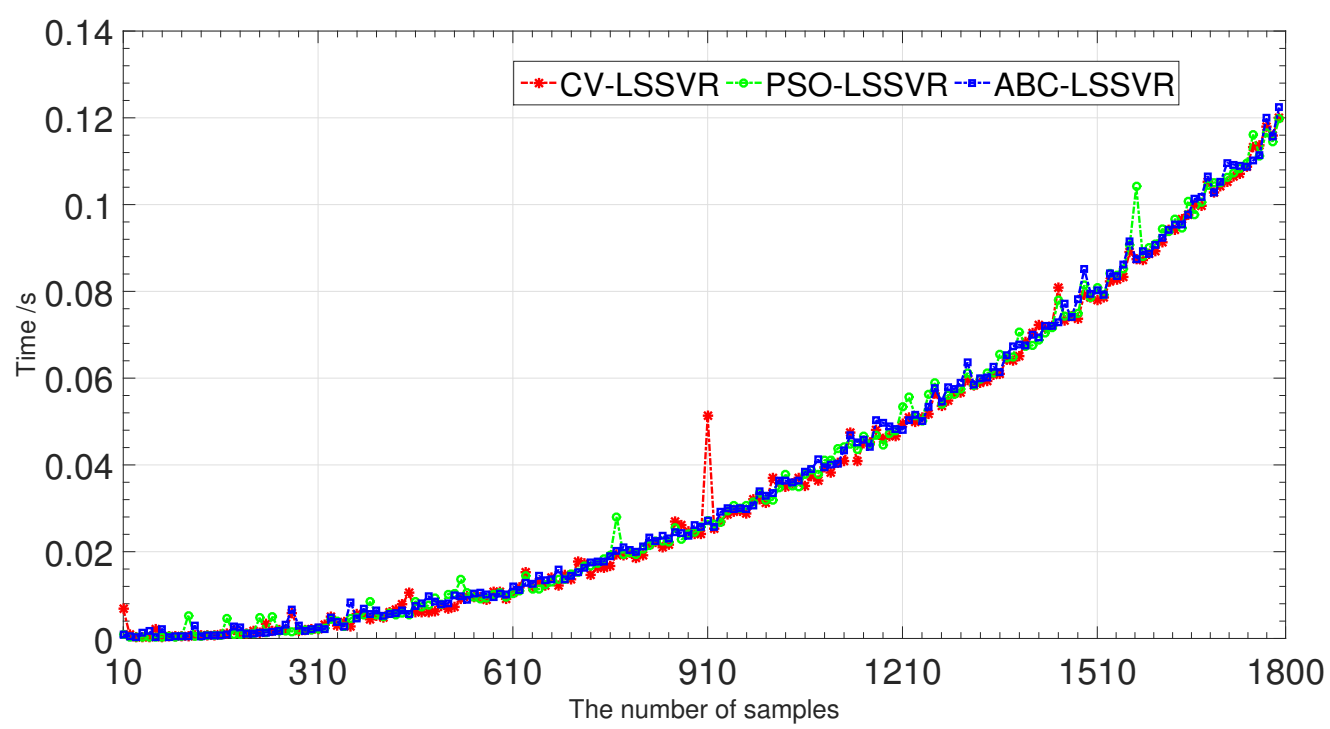

Figure 7.4.7: The time consumed by the three estimation methods on identifying the surge model.

and estimated parameters of all identified surge models is obvious within the first 350 samples but becomes stable soon after. Compared to CV-LSSVR and PSO-LSSVR, ABC-LSSVR mostly provides the identified surge model with the lowest MSE. For the identification of the steering model, the performance of PSO-LSSVR is not so desirable as that of CV-LSSVR and ABC-LSSVR. This is due to PSO-LSSVR is prone to fall into local optima. Through the identified steering models of CV-LSSVR and ABC-LSSVR, one can notice that MSE of the identified sway models and the identified yaw models have no apparent variations after the first 630 samples and the first 230 samples. Recall that the time complexity of ABC-LSSVR increases along with the increasing of the number of samples. Therefore, the suitable selection of samples for efficient identification of the surge model, the sway model, and the yaw model is 350,630 and 230 , respectively. 

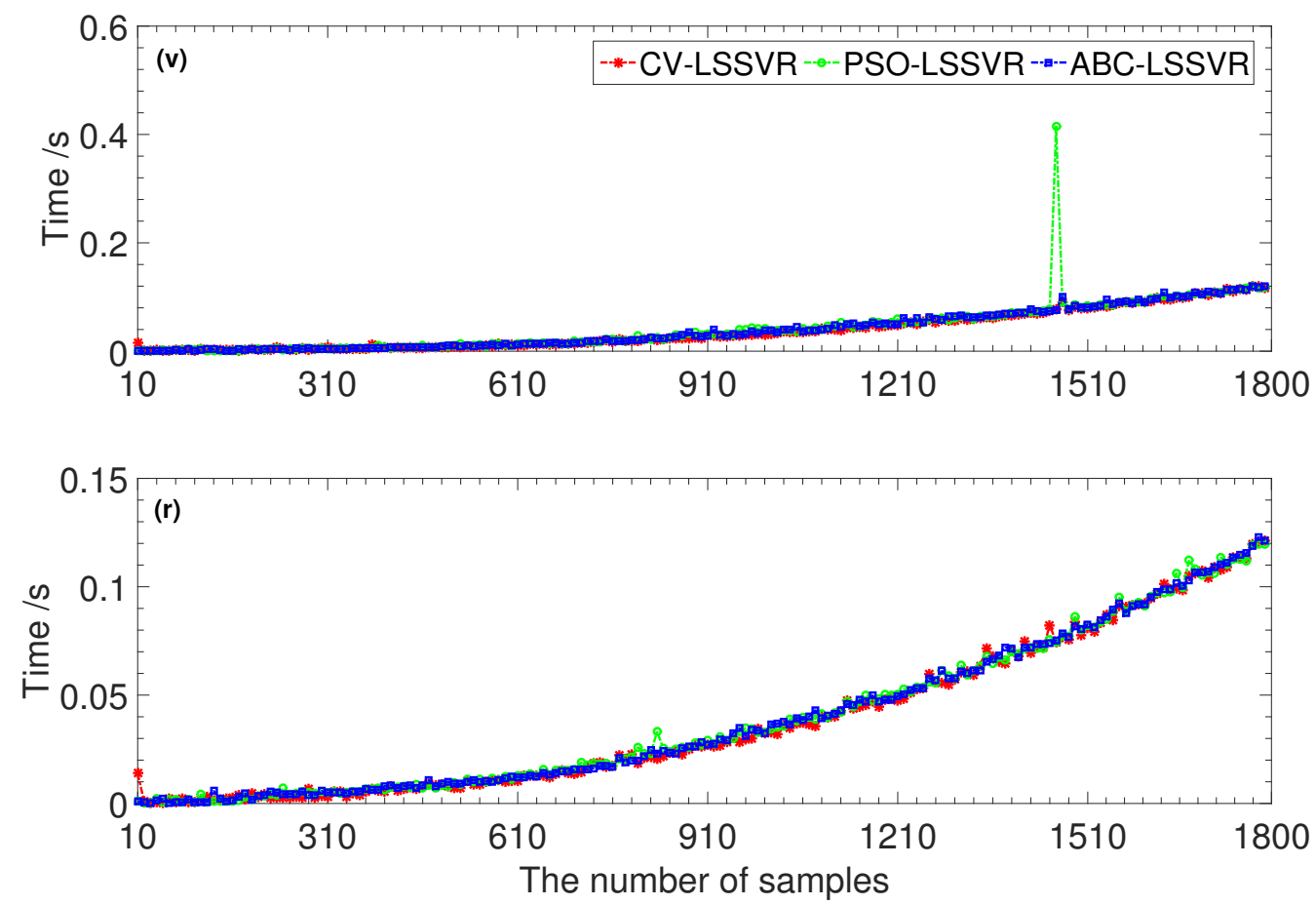

Figure 7.4.8: The time consumed by the three estimation methods on identifying the steering model. The top sub-figure presents the cost time of the sway model, the bottom subfigure means the cost time of the yaw model.

\subsection{Case 4: Experimental Validation of the Further Simplified Dynamic MODEL}

The study undertook in this part are additional tests on verifying if the further simplified ship dynamic model obtained through simulation study is also useful for describing the dynamics of a real unmanned surface vessel, called I-Nav-II vessel. The optimized LS-SVR based on ABC in the combination of the experimental data stemmed from a set of full-scale trails is used to identify the model of I-Nav-II vessel.

\subsubsection{Data Acquisition and Processing}

The tests on the I-Nav-II vessel were carried out in relatively calm water, i.e., the East Lake of China. The ship is presented in Figure 7.5.1, which is powered by two batteries with the maximum voltage of $48 \mathrm{~V}$. The particulars of this ship are the length of $4.15 \mathrm{~m}$, the breath of $1.6 \mathrm{~m}$, 


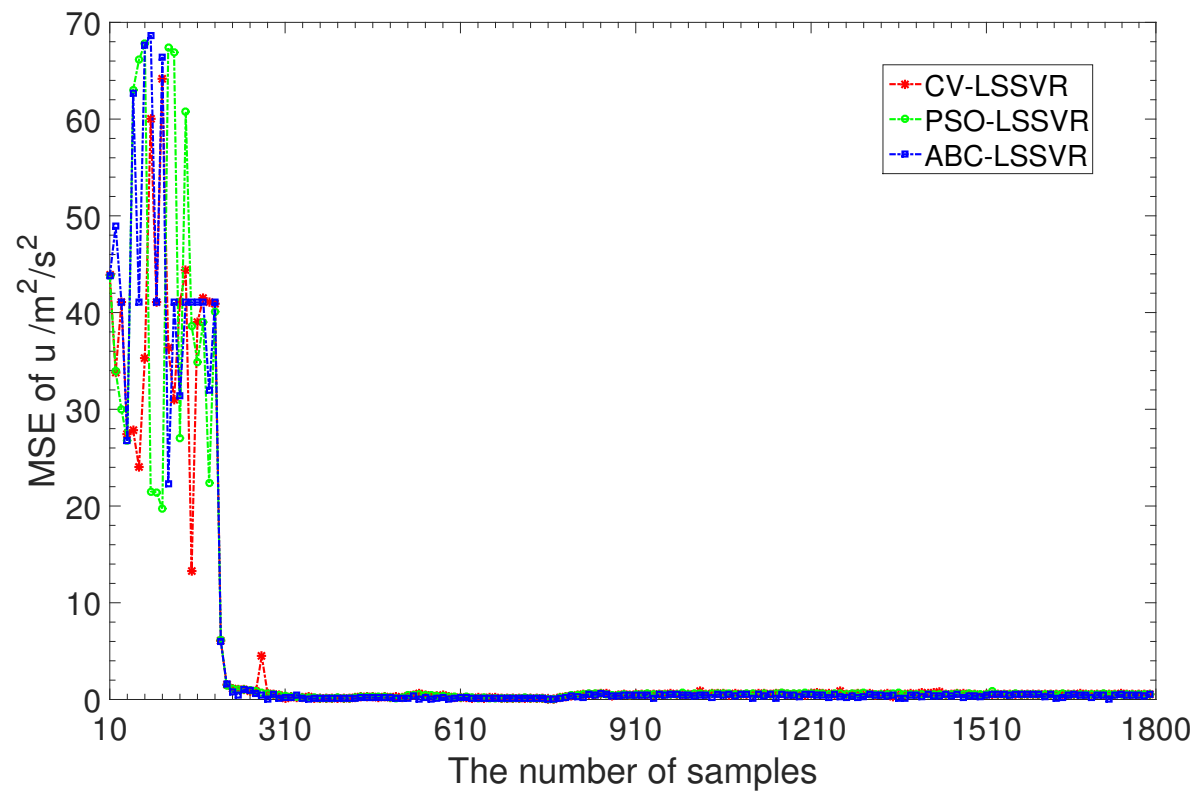

Figure 7.4.9: The MSE of the three identified surge model.

the draught varying from 0.3 to $0.5 \mathrm{~m}$, and the rudder deflection set at $\pm 25^{\circ}$.

The data obtained are less desirable than the ones acquired in the previous section. Two reasons can be explained for this. Firstly, the tests were carried out in relatively calm water, but there existing noticeable environmental disturbances induced by wind. Secondly, the measurement devices used to record experimental data are limited. Only the position, yaw rate, heading angle, and actual rudder angle can be measured by a global positioning system ( $\mathrm{R}_{93} \mathrm{~T}$ GPS), an inertial measurement unit (Mit-G-700 IMU), a compass ( $\mathrm{HCM}_{35} 6 \mathrm{~B}$ compass) and a rudder indicator, respectively. The revolutions of the propeller are impossible to be measured. Hence, the surge model of the I-Nav-II vessel is unable to be determined in this case study.

The available data are extracted from a set of zigzag maneuvers carried out under the condition of a steady forward speed at around $3 \mathrm{~m} / \mathrm{s}$. The median filter is utilized to eliminate disturbances and noises corrupted in the measured data. After the filtering, the data is restored in the interval of $1 s$. The speeds containing surge speed and sway speed are unavailable but calculated by taking the derivatives of the measured positions. 142 samples extracted from $10^{\circ} / 10^{\circ}$ zigzag maneuver are regarded as the training data to identify the simplified steering model for the I-Nav-II vessel. The training data including yaw rate, actual rudder angle, heading angle and sway speed are illustrated in Figure 7.5.2. The rest experimental data stemmed from $20^{\circ} / 20^{\circ}$ and $25^{\circ} / 25^{\circ}$ zigzag maneuvers are treated as validation data to validate the identified model. 

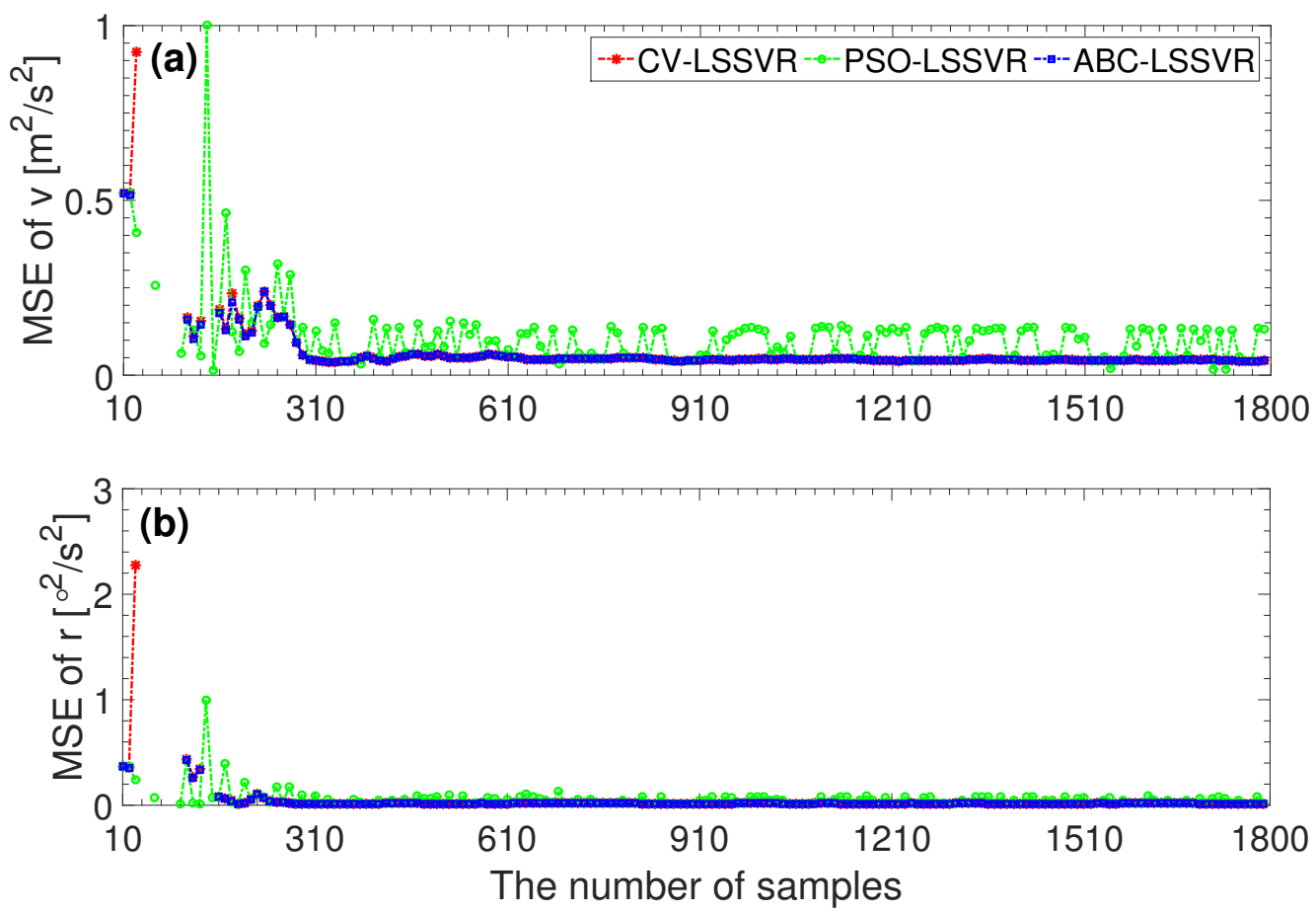

Figure 7.4.10: The MSE of the three identified further simplified steering model.(a) the MSE of the identified sway models. (b) the MSE of the identified yaw models.

\subsubsection{IDENTIFICATION Results ANd Validation}

After processing the identification procedure, the steering model of the I-Nav-II vessel is obtained. The model expressed with estimated parameters is given as

$$
\begin{aligned}
& \dot{v}=-0.5706 v-1.1529 r+1.4889|v| r-0.0775 \delta \\
& \dot{r}=-0.1386 v-0.5113 r-1.4883|v| r+0.2839 \delta
\end{aligned}
$$

Then the identified steering model is validated by comparing experimental maneuvers with predictions of $20^{\circ} / 20^{\circ}$ zigzag maneuver shown in Figure 7.5.3 and $25^{\circ} / 25^{\circ}$ zigzag maneuver in Figure 7.5.5, respectively. The tendency of the experimental maneuvers and predictions fits quite well. Although errors can be observed in the predictions as shown in Figure 7.5.4 and Figure 7.5.6, it attributes negligible errors in estimating the parameters of the steering model. The identified steering model is still ideal to describe the steering dynamics of the I-Nav-II vessel for 

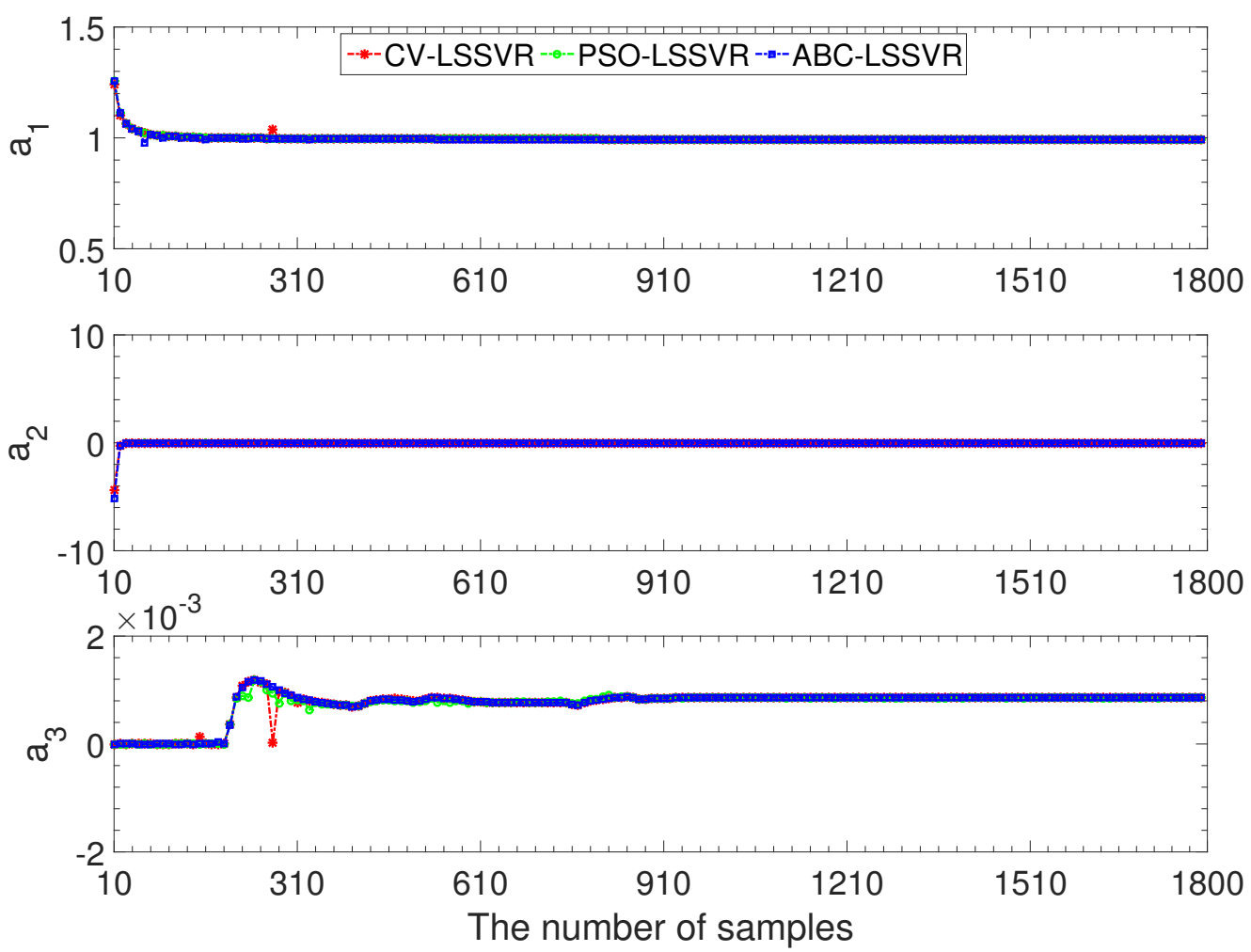

Figure 7.4.11: The estimated parameters of the three identified surge models. The top subfigure is the parameter $a_{1}$, the middle sub-figure donates the parameter $a_{2}$, the bottom subfigure means the parameter $a_{3}$.

practical applications.

\subsection{Summary of Objective Coverage and Requirement Fulfillment}

The proposed SIMSD system has been evaluated through case studies in which the required ship dynamic models, methods, purposes and results are explained in detail. According to the evaluation results, the sub-questions and the coverage of corresponding objectives are judged, at the same time the fulfillment of requirements is discussed.

\section{On Sub-Question 1: How to simplify the complex dynamic model for ships?}

Through the overview of related work on ship dynamic model, the advantages and disadvantages of each model have been analyzed, which are treated as the criterion for the decision of the 6 DOF fundamental model. The vectorial representation model is selected 

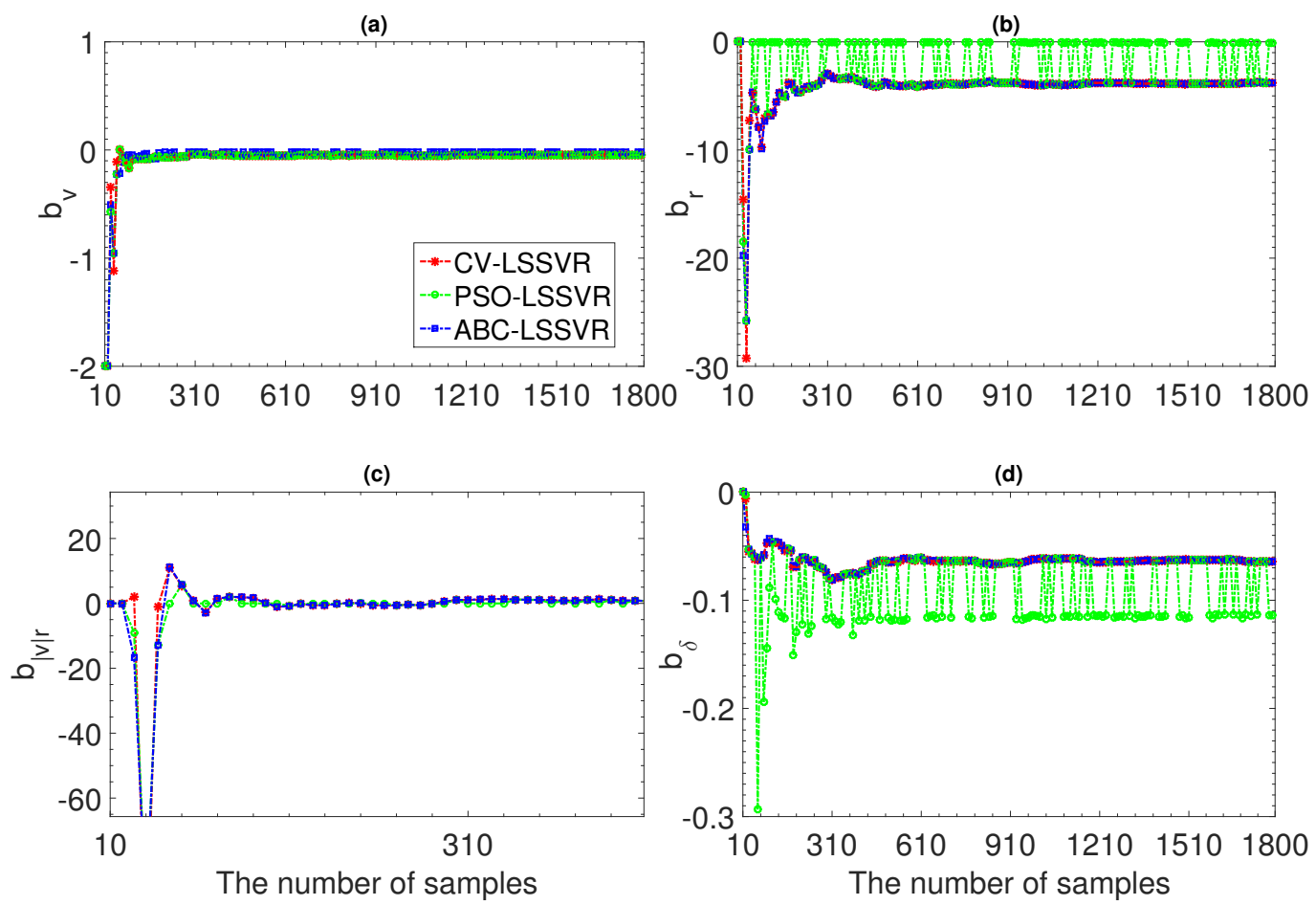

Figure 7.4.12: The estimated parameters of the three identified further simplified sway models.

(objective 1). This model expressed in the vector-matrix form is easy-understanding and applicable for the model simulation to investigate underlying characteristics of ship dynamics. The common version of a 3 DOF model simplified from the 6 DOF vectorial representation model is further simplified based on a series of reasonable assumptions. Besides, some terms with low sensitivity to the response of the ship are also removed. The simplified model is firstly tested on a large container ship for which the dynamics are modeled by a 4 DOF model with much higher complexity than the proposed simplified model. The results reveal that the model is simple but inaccurate to describe the dynamics of the container ship, which motivates the further modification of the simplified model. The further simplified steering model in which each equation is expressed with five terms is validated through studies on the container ship and the I-Nav-II vessel (objective 2).

The validated model expressed in a relatively simple form where the equation for capturing surge dynamics given in four terms and each equation of steering model built by five terms satisfies the second requirement (R2: The model is expressed in a simple form with low order and a few terms.). As this model is simplified from the 3 DOF vectorial representations, each 
(a)

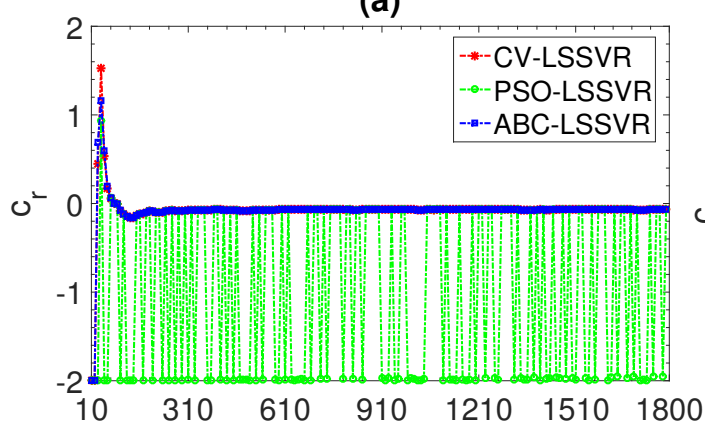

(c)

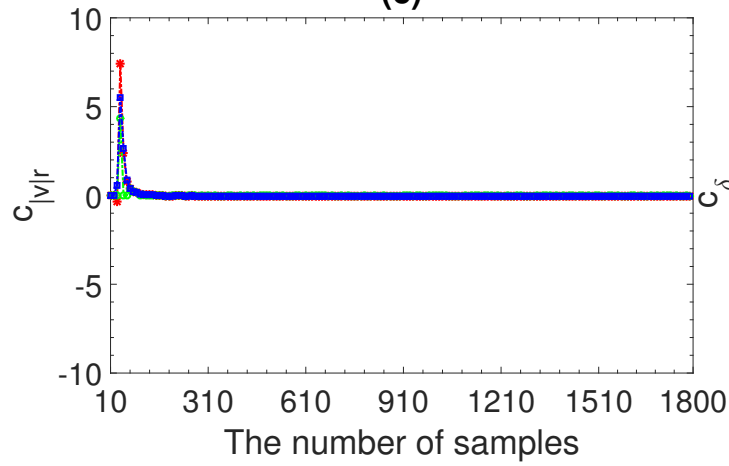

(b)

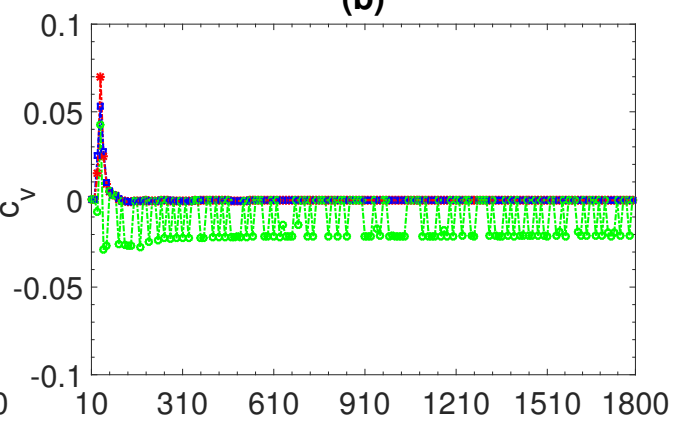

(d)

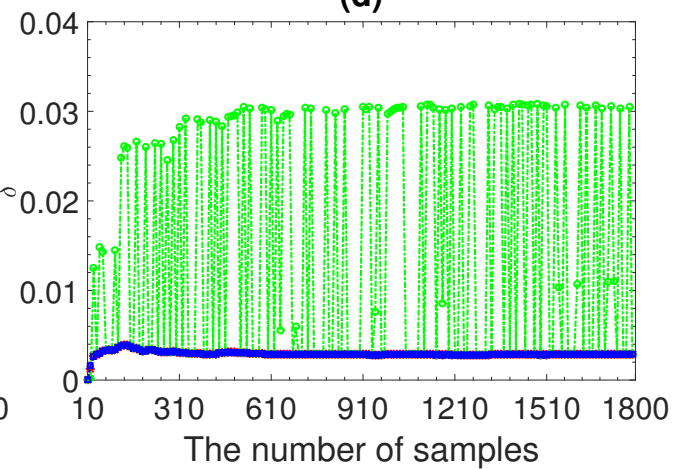

Figure 7.4.13: The estimated parameters of the three identified further simplified yaw models. (a) parameter $c_{r}$, (b) parameter $c_{v}$, (c) parameter $c_{|v| r}$, (d) parameter $c_{\delta}$.

term in the model has a manifest physical interpretation (R1: Each term in the model has a manifest physical interpretation.) except for the third order term added to the surge model under the consideration of the suggestion about improving model accuracy from (Skjetne et al. 2004). However, through comprehensively analyzing the estimated parameter of this third order term and the prediction errors of the surge model, the third term is not so critical up to this thesis to improve the surge model accuracy. Therefore, with the relatively high certainty limited to the present study, the first requirement (R1: Each term in the model has a manifest physical interpretation.) is fulfilled. In the chapter regarding the modeling of ship dynamics, the parameter identifiability problem is handled by moving parameters of the left hand of models to the right hand to further merge parameters together to be one integrated parameter. After this process, the number of parameters is equal to that of inputs. The simulation study on a container ship indicates the effects of parameter drift existing in the identification results, which is effectively mitigated by reducing the terms of the simplified dynamic model. The further considerations on simplifying the steering model are not only boosting the identifiability of the dynamic model ( $\mathbf{R}_{3}$ : The model is identifiable.) but also extenuating negative influences of 


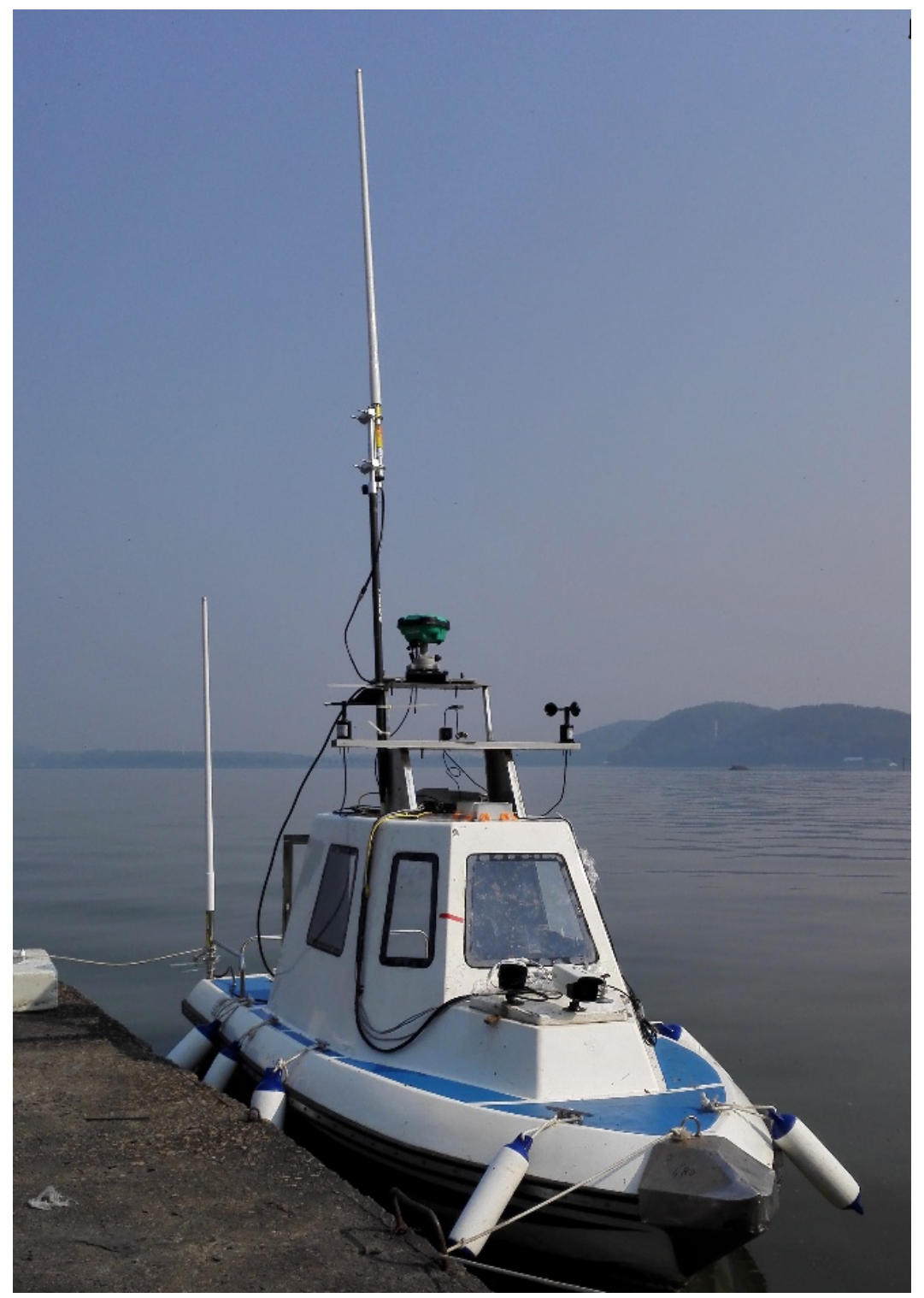

Figure 7.5.1: The I-Nav- II vessel.

parameter drift on estimated parameters. The fourth requirement ( $\mathbf{R}_{4}$ : The model guarantees acceptable accuracy in describing ship dynamics.) is achieved by analyzing the indicator MSE of the predicted surge speed, the predicted sway speed, and the yaw rate respectively. For a container ship, MSE of the predicted surge speed of a straight line maneuver is $0.1289 \mathrm{~m}^{2} / \mathrm{s}^{2}$ which is less than $0.5 \mathrm{~m}^{2} / \mathrm{s}^{2}$ within the accepted range, and MSEs of the sway speed and yaw rate of a $20^{\circ} / 20^{\circ}$ zigzag maneuver are $0.0472 \mathrm{~m}^{2} / \mathrm{s}^{2}$ and $0.0162^{0^{2}} / \mathrm{s}^{2}$, respectively. Besides the simulation study on the container ship, the experimental study on the I-Nav-II vessel shows MSEs of the sway 

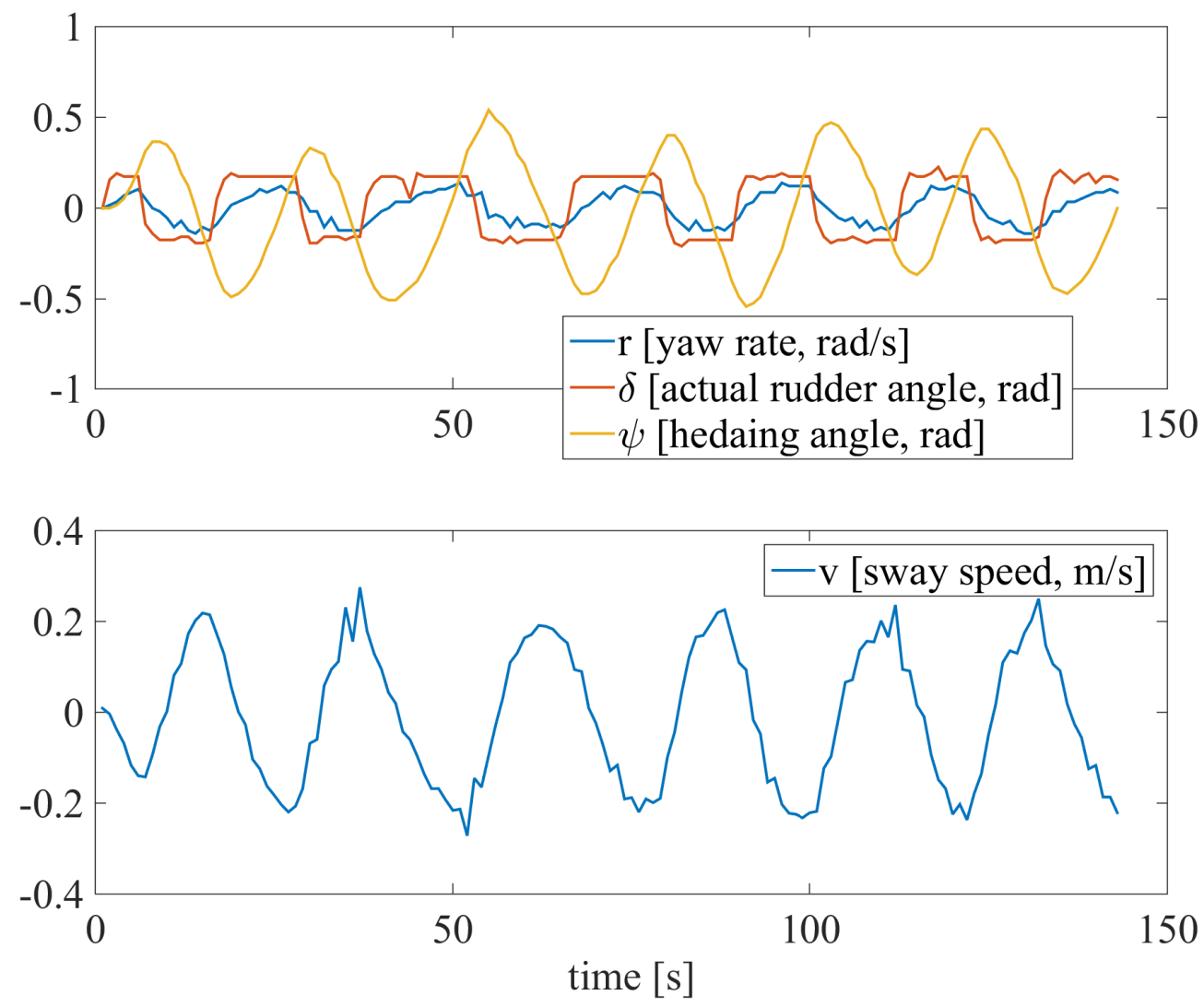

Figure 7.5.2: Experimental data of the $10^{\circ} / 10^{\circ}$ zigzag maneuver on the I-Nav-II vessel.

speed of the $20^{\circ} / 20^{\circ}$ and $25^{\circ} / 25^{\circ}$ zigzag maneuver are $0.0035 \mathrm{~m}^{2} / \mathrm{s}^{2}$ and $0.0017 \mathrm{~m}^{2} / \mathrm{s}^{2}$ respectively, and the corresponding MSEs of the yaw rate are $3.6220^{0^{2}} / s^{2}$ and $3.9294^{0^{2}} / s^{2}$, respectively.

On Sub-Question 2: How to estimate the parameters in the simplified ship dynamic model?

Case study 1 on comparing commonly-used LS with LS-SVR in identify response models proves LS-SVR based identification method is a powerful selection (objective 3). LS-SVR based identification method just works with a finite set of data, has no requirement of the initial estimation of model parameters, and is not so sensitive to the outliers. However, one deficiency of LS-SVR is the particular setting for its regularization parameter, which is remedied by using CV, PSO, and ABC (objective 4). The LS-SVR with regularization parameter optimized by ABC shows superior performance on model identification over the other two methods. The simulated maneuvering data generated by a 4 DOF model with predetermined parameters in the combination of the optimized LS-SVR are used to test the effectiveness of the simplified model to 

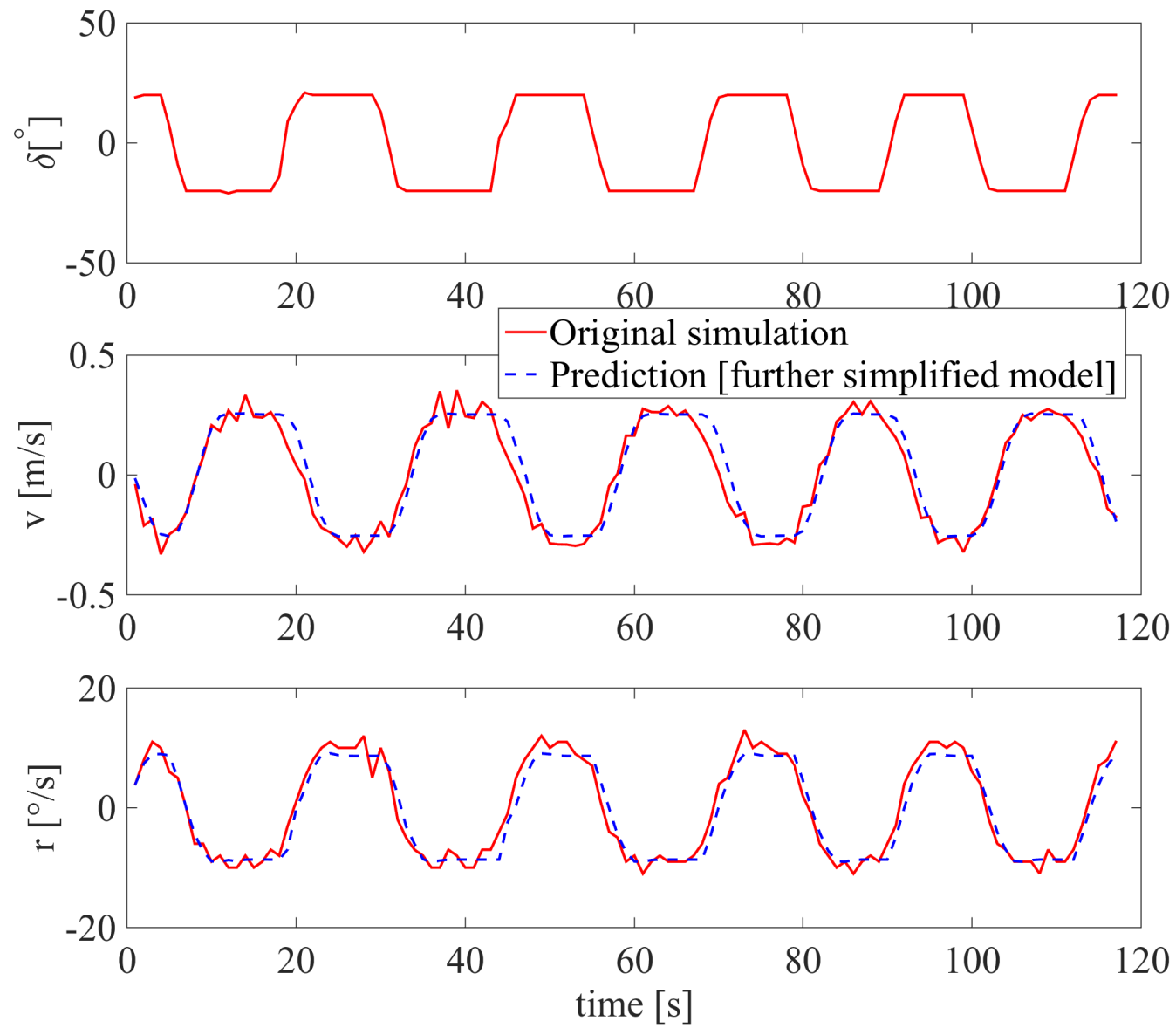

Figure 7.5.3: Predictions of the $20^{\circ} / 20^{\circ}$ zigzag maneuver.

describe the dynamics of a large container ship. Furthermore, the simplified dynamic model is also identified by using ABC-LSSVR incorporated with experimental data extracted from the maneuvers of the I-Nav-II vessel (objective 5).

Even though the experimental data of the I-Nav-II vessel are adopted, the developed identification method, i.e., ABC-LSSVR, can identify the dynamic model with accepted accuracy, which proves ABS-LSSVR method is robust to defend disturbance and noise effects (R5: The identification method is robust enough.). The hybrid ABC-LSSVR method is absorbing advantages of LS-SVR as one of the machine learning techniques and $A B C$ as one of the intelligent algorithms to identify the ship dynamic model with no requirement of predetermined parameters, from which the sixth requirement is achieved (R6: The identification method has no sensitivity to the predefined values of the parameters which are required to be 

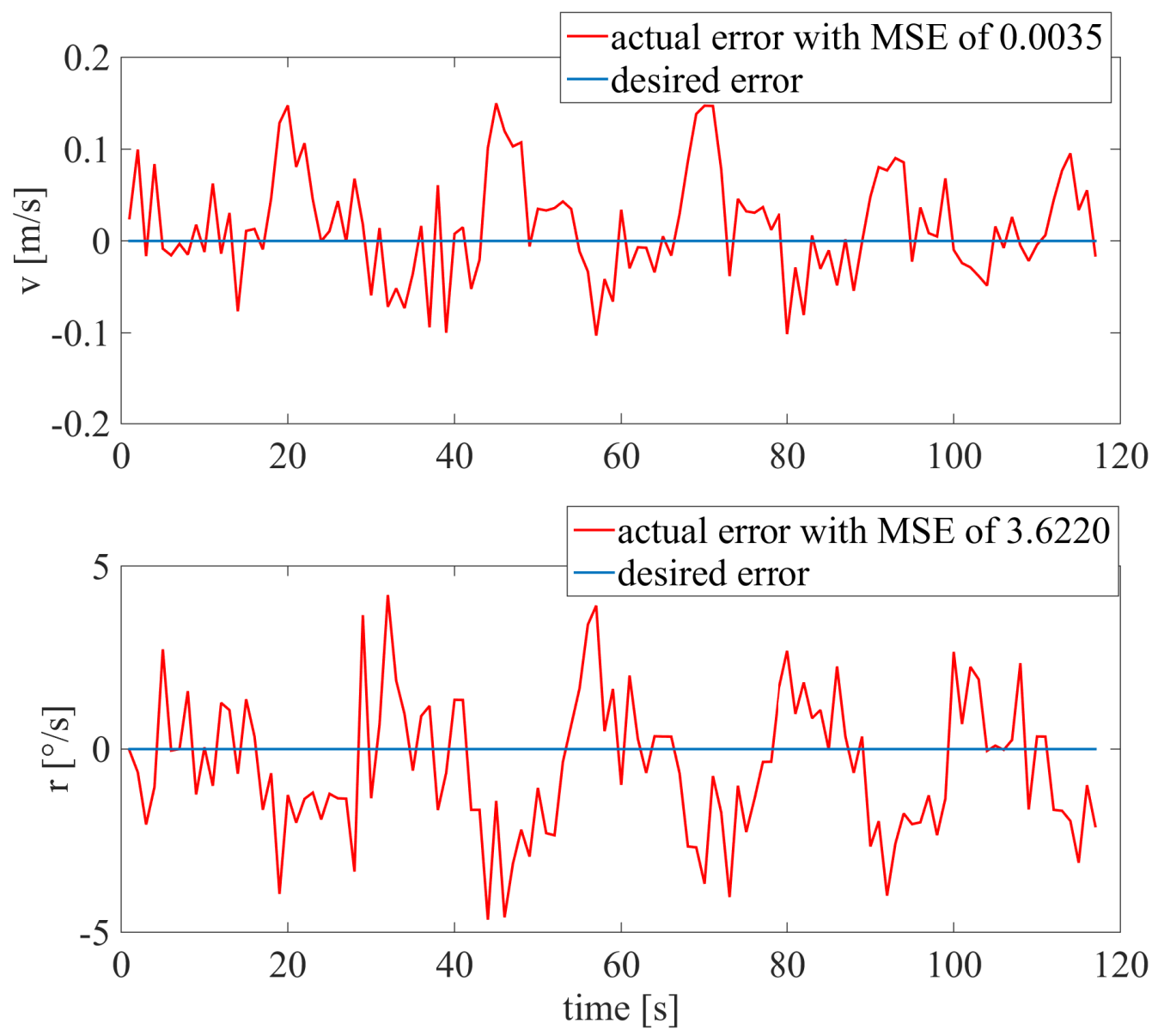

Figure 7.5.4: Errors of the $20^{\circ} / 20^{\circ}$ zigzag maneuver.

estimated.). The particular requirement of LS-SVR based identification method, which is the particular setting for the regularization parameter, is fitted by applying $\mathrm{ABC}$. This process guarantees superior identification results of ABC-LSSVR over that of the CV-LSSVR and PSO-LSSVR. Even though ABC-LSSVR demonstrates outperformance while regarded as an identifier, the global optimal identification results can be guaranteed with high probability. Therefore, the seventh requirement ( $\mathbf{R}_{7}$ : The identification method guarantees the global optimal solution.) is fulfilled within the relatively high certainty limited to the present study. From the results of the complexity analysis of the identification methods, we can see that the eighth requirement (R8: The identification method should be of low complexity.) is achieved. The time complexity of ABC-LSSVR increases along with the increase of the number of samples, but the suitable number of samples for efficient identification of the surge model, the 

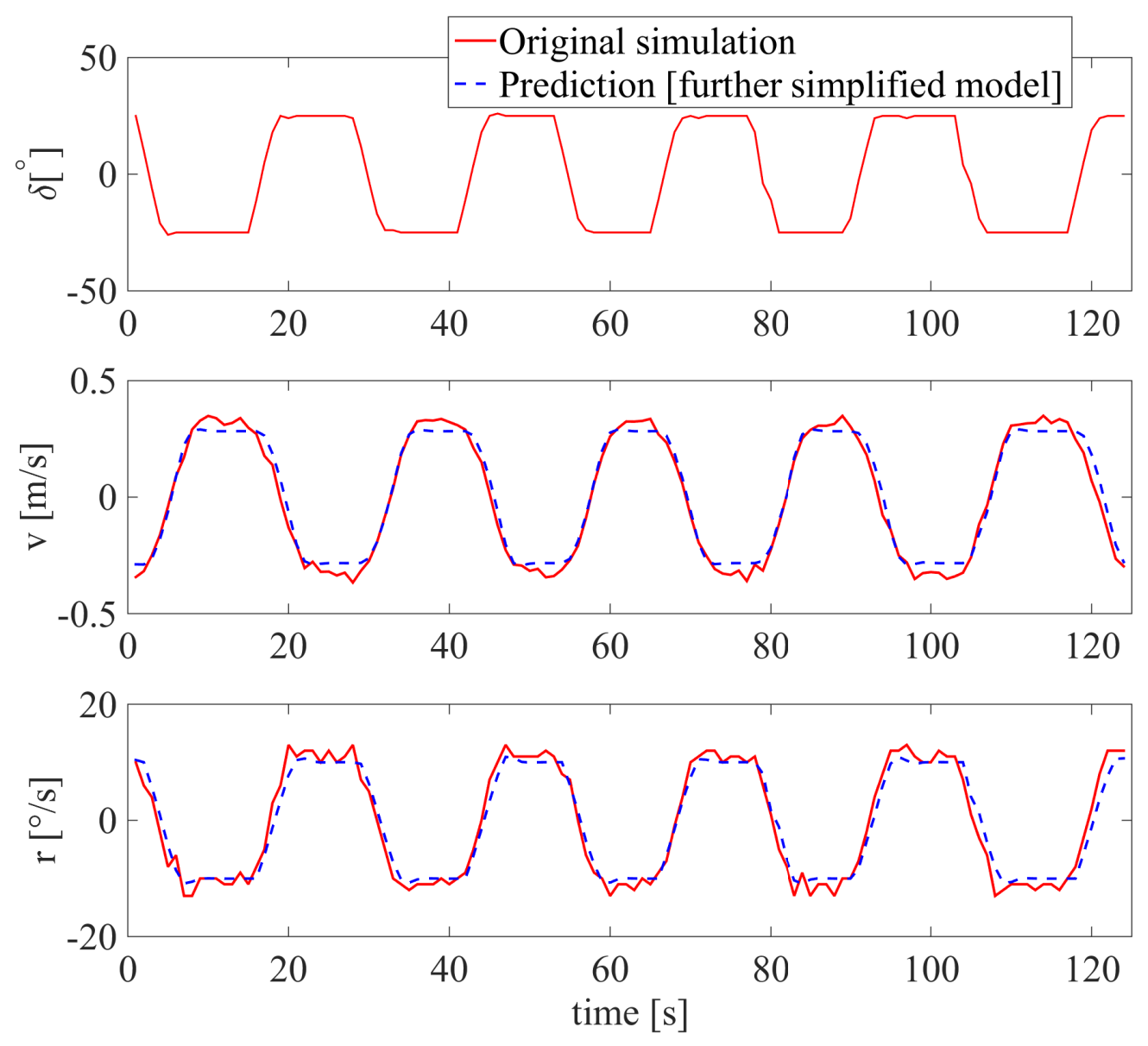

Figure 7.5.5: Predictions of the $25^{\circ} / 25^{\circ}$ zigzag maneuver.

sway model and the yaw model for the container ship which is 350,630 and 230 respectively reveals the low computational cost of the identification procedure.

In summary, the evaluation results have developed answers to the sub-questions, and the objectives in this thesis have been achieved. With the relatively high certainty limited to the present study, requirements are fulfilled. 

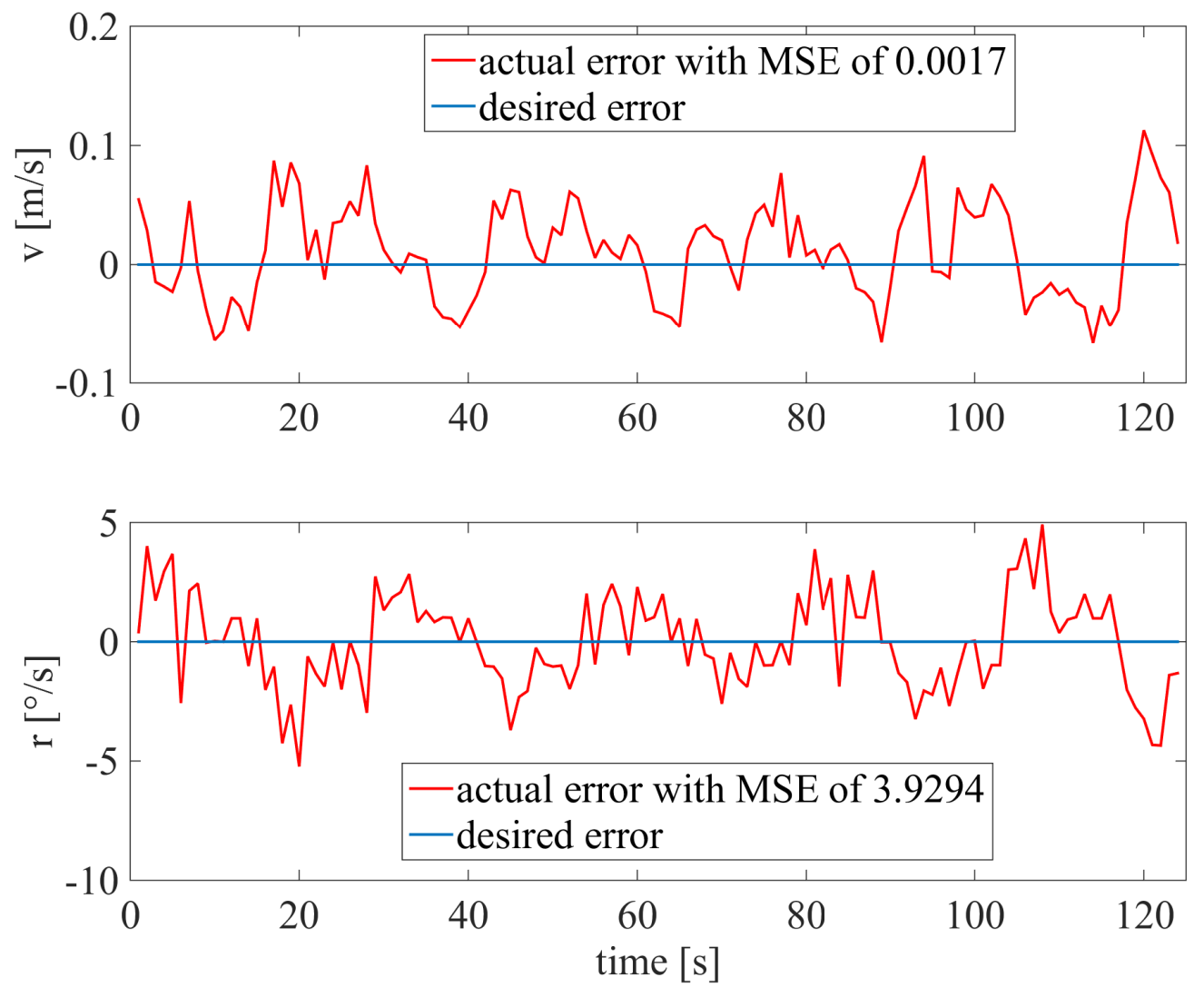

Figure 7.5.6: Errors of the $25^{\circ} / 25^{\circ}$ zigzag maneuver. 


\section{8 \\ Conclusions}

A model with relatively low complexity and high accuracy is desirable for describing ship dynamics, which can attenuate the difficulty of model identification and model simulation. This thesis makes great attempts to answer the research question of How to derive a simplistic model in describing the dynamics of ships for maritime traffic simulation. The derived objectives are achieved throughout this thesis to answer the focused question. The requirements derived are fulfilled in the evaluation chapter.

This chapter concludes the work and findings throughout this thesis in Section 8.1, as well as outlines the potential future research points based on current achievements in Section 8.2.

\subsection{SUMMARY}

Literature review in Chapter 2 on the state-of-the-art ship dynamic models, encompassing the Abkowitz model, MMG model, Nomoto model, and Fossen's vectorial model reveals that the determination of the fundamental model for modification and simplification is Fossen's vectorial model. This is due to the effects of ship hull, rudder, propeller and their interference are completely expressed in matrix-vector form. This form is not only intuitive for analyzing the 
properties of a ship using passivity and stability analysis but also easy for the local changes and model simulation.

By comparing the related work with the aroused objectives, shortcomings are observed. Based on the observed shortcomings, requirements on the modeling of ship dynamics and identification methods are created in Chapter 3.

The 6 DOF vectorial model is simplified into a 3 DOF dynamic model by employing pieces of reasonable assumptions. The actuator forces and moments acting on the ship in the $3 \mathrm{DOF}$ dynamic model are given by the function of propeller thrust and rudder angle. In order to reduce the complexity of the 3 DOF dynamic model, the coupling interactions between surge motions and steering motions are assumed to be ignorable. Consequently, the decoupled surge model and steering model with relatively low complexity are obtained, as presented in Chapter 4.

To decide a powerful identification method for estimation of parameters in the simplified ship dynamic model, the comparison between commonly-used LS and LS-SVR is executed on identifying the simple Nomoto models containing the first order liner and nonlinear Nomoto models. The results obviously demonstrate that LS-SVR outperforms LS. This is due to several aspects. LS aiming at minimizing a squared norm makes it sensitive to the outlier, which means that if an outlier exists in the training data, identification results of LS would be affected adversely. On the contrary, LS-SVR simultaneously achieves both structural risk minimization and empirical risk minimization when doing the identification. This characteristic sufficiently decreases the sensitivity of LS-SVR to the outlier.

Apart from the above-mentioned characteristics, LS-SVR can work with a finite set of data and require non-predefined values of parameters contained in the model. Benefiting from LS-SVR, a solution is developed to overcome the problem of some identification methods, e.g., RLS, requiring initial value definition for identification. To provide initial values of parameters for RLS, 200 samples in the interval of 3 are used by LS-SVR. From the results, the fluctuation of convergence of RLS is more intense than that of the combined LS-SVR and RLS method. Besides, the parameter initially estimated by LS-SVR close to the true value has high accuracy. It is indicated that the developed solution is sufficient to improve the performance of RLS on parameter estimation.

To guarantee the performance of LS-SVR on generating the globally optimal solution, the tuning of the regularization parameter in LS-SVR is executed by using ABC. Comparing the optimization performance of $\mathrm{PSO}, \mathrm{CV}$, and $\mathrm{ABC}$ proves that $\mathrm{ABC}$ can present superior performance over the other two methods with the advantage of fewer control parameters. $\mathrm{CV}$ as a trial and error method is a little time-consuming to find a suitable value for the regularization 
parameter. The result is not so optimal as the optimization results of PSO or ABC. PSO can find the global optimum for LS-SVR, but sometimes it falls into a local optimum. Comparatively, $\mathrm{ABC}$ shows relatively stable global optimization ability during the selection of the regularization parameter of LS-SVR. As a consequence, CV could be an option to select a regularization parameter for LS-SVR based on reliable experiences, but ABC is a superior alternative to optimize LS-SVR.

Predictions of the simplified ship dynamic model with estimated parameters poorly fit the validation data, which motivates special considerations on further reducing the terms of the simplified ship dynamic model, in particular, the steering model. By recombining terms extracted from the simplified steering model, a series of the reconstructed models with lower complexity is obtained. These models are identified by using the ABC-LSSVR method in conjunction with simulated data of a container ship. Finally, the model generating the lowest MSE is selected as the further simplification steering model which is extensively tested on the experimental data stemmed from zigzag maneuvers of a small size ship. Testes on different types of ships including a large container ship and a small vessel demonstrate that the further developed steering model is valid to capture the steering dynamics of different types' ships. Following the validation of the further simplified ship dynamic model, the time complexity of ABC-LSSVR in identifying the valid simplified model is analyzed to conclude that the cost time increases along with the increase of the number of samples which is consistent with the analysis of the time complexity of $O\left(N^{2}+l^{3}\right)$.

Through the evaluation results, the created requirements are fulfilled. The proposed approach on system identification-based modeling of ship dynamics is feasible. The obtained dynamic model is simple enough to capture the dynamics of different types' ships.

\subsection{OUTLOOK}

The derived objectives and requirements are covered through the evaluation of applying optimized SVR to identify the simplified dynamic model for different types of ships. To enhance more contributions based on present achievements, the forthcoming points deserved to be studied are presented as follows.

- Even though the further simplified steering model has been validated through the simulated data generated by a large container ship and experimental data conducted by a real vessel, devices are needed to be mounted on the ship to collect benign experimental data used to verify and validate the simplified surge model. Besides, full-scale trails should be carried 
out in the calm environment where the disturbances induced by the wave, current and wind are ignorable so that the tests on the proposed identification method and simplified ship dynamic model would be more effective.

- The combined LS-SVR and RLS has been proved effective. But it is still worthy to expand the benefit of LS-SVR about supplying predefined values of parameters to more recursive identification methods, e.g., EKL, ML.

- For LS-SVR based identification method, this thesis applies the linear kernel function due to the ship dynamic model is linear with respect to the parameters, but in the future, the performance of LS-SVR in conjunction with the other kernel functions on model identification is worthy to be investigated.

- Applications of either offline identification or online identification exist in marine engineering. Thus, the extensive study on performing online identification by using the modified LS-SVR in conjunction with ABC could be contemplated, which would be no easy task. Noticeably, real-time data acquisition and data preprocessing are certainly necessitated. 


\section{A Appendix}

\section{A.1 Least SQuare Method}

Due to the simple structure and effectiveness, LS is regarded as a classic system identification technique. LS is proposed based on the experience risk minimization. Suppose a system with multiple-input-single-output (MISO) expressed as

$$
Y=X \theta\left(X \in R^{m}, Y \in R\right)
$$

where $X$ are input samples, $Y$ are samples of output, $\theta$ donate the parameters required to be estimated, $m$ presents the dimension of the input samples.

The parameters can be estimated by $\hat{\theta}=X^{-1} Y$. Errors of the identified system are calculated by $\varepsilon=Y-X \hat{\theta}$. Afterward, the objective function is given as

$$
J=\sum_{i=1}^{n} \varepsilon_{i}^{2}=\varepsilon^{T} \varepsilon
$$

in which $n$ is the number of samples. 
In order to minimize the object function, the (A.2) is partially differentiated and the differential results are set as zero.

$$
\left.\frac{\partial J}{\partial \theta}\right|_{\theta=\hat{\theta}}=\frac{\partial(Y-X \hat{\theta})^{T}(Y-X \hat{\theta})}{\partial \theta}=-2 X^{T} Y+2 X^{T} X \hat{\theta}=0
$$

Then the LS approximation of (A.1) is obtained by solving (A.3)

$$
\hat{\theta}=\left(X^{T} X\right)^{-1} X^{T} Y
$$

\section{A.2 Recursive Least Square Method}

In this part, RLS is briefly introduced. RLS is developed for on-line parametric identification based on an off-line method, i.e., LS. Given a system organized with a linear regression form using a model parameter vector $\theta$, a lagged input-output data vector $X(k)=\left[x^{T}(1), x^{T}(2), \cdots, x^{T}(k)\right]$, and an unspecified noise process $v(k)$ as follows

$$
y(k)=X^{T}(k) \theta+v(k)
$$

Then, parameters in $\theta$ are estimated by the followings

$$
\left\{\begin{array}{c}
\hat{\theta}(k)=\hat{\theta}(k-1)+K(k)\left[y(k)-x^{T}(k) \hat{\theta}(k-1)\right] \\
K(k)=P(k-1) x(k)\left[I+x^{T}(k) P(k-1) x(k)\right]^{-1} \\
P(k)=\left[I-K(k) x^{T}(k)\right] P(k-1)
\end{array}\right.
$$

\section{A.3 A Linear Steering Model}

Assume that the ship surge speed is constant $\left(u_{\mathrm{o}}\right)$, the steering dynamics of the Merchant ship Mariner class can be described as (Åström and Källström 1976)

$$
\left[\begin{array}{ccc}
m^{\prime}-Y_{\dot{v}}^{\prime} & m^{\prime} x_{G}^{\prime}-Y_{\dot{r}}^{\prime} & 0 \\
m^{\prime} x_{G}^{\prime}-N_{\dot{v}}^{\prime} & I_{Z}^{\prime}-N_{\dot{r}}^{\prime} & 0 \\
0 & 0 & 1
\end{array}\right]\left[\begin{array}{c}
\dot{v}^{\prime} \\
\dot{r}^{\prime} \\
\dot{\psi}^{\prime}
\end{array}\right]=\left[\begin{array}{ccc}
Y_{v}^{\prime} & Y_{r}^{\prime}-m^{\prime} & 0 \\
N_{v}{ }^{\prime} & N_{r}^{\prime}-m^{\prime} x_{G}^{\prime} & 0 \\
0 & 1 & 0
\end{array}\right]\left[\begin{array}{c}
v^{\prime} \\
r^{\prime} \\
\psi^{\prime}
\end{array}\right]+\left[\begin{array}{c}
Y_{\delta}^{\prime} \\
N_{\delta}^{\prime} \\
0
\end{array}\right] \delta^{\prime}
$$

where $m$ is the non-dimensional mass of the ship, $x_{G}^{\prime}$ is the non-dimensional longitude coordinate of the ship's center of gravity, $I_{Z}^{\prime}$ is the non-dimensional inertia moment about $z$-axis, $\dot{v}^{\prime}$ and $\dot{r}^{\prime}$ are non-dimensional small perturbations respectively, $v^{\prime}$ is the non-dimensional sway linear velocity, 
$\dot{\psi}^{\prime}$ and $r^{\prime}$ are non-dimensional yaw rate, $\psi^{\prime}$ is the non-dimensional heading angle, $\delta^{\prime}$ is the rudder angle, $Y_{\dot{v}}^{\prime}, Y_{\dot{r}}^{\prime}, Y_{\delta}^{\prime}, Y_{v}^{\prime}$ and $Y_{r}^{\prime}$ are the respective hydrodynamic coefficients of the sway motion, $N_{\dot{v}}^{\prime}$, $N_{\dot{r}}^{\prime}, N_{\delta}^{\prime}, N_{v}^{\prime}$ and $N_{r}^{\prime}$ are respective hydrodynamic coefficients of the yaw motion, and $\dot{v}^{\prime}=\frac{\dot{\nu} L}{U^{2}}$, $\dot{r}^{\prime}=\frac{i L}{U^{2}}, \dot{\psi}^{\prime}=\frac{\dot{\psi} L}{U}, v^{\prime}=\frac{v L}{U}, r^{\prime}=\frac{r L}{U}, \delta^{\prime}=\delta, U=\sqrt{u_{\mathrm{o}}^{2}+v^{2}}$.

The normalized equations of motion, i.e., (A.7), are easily converted to standard state-space notations by solving for the derivatives $\dot{v}^{\prime}$ and $\dot{r}^{\prime}$. The state-space expression is given as

$$
\left[\begin{array}{c}
\dot{v}^{\prime} \\
\dot{r}^{\prime} \\
\dot{\psi}^{\prime}
\end{array}\right]=\left[\begin{array}{ccc}
a_{11} & a_{12} & 0 \\
a_{21} & a_{22} & 0 \\
0 & 1 & 0
\end{array}\right]\left[\begin{array}{c}
v^{\prime} \\
r^{\prime} \\
\psi^{\prime}
\end{array}\right]+\left[\begin{array}{c}
b_{11} \\
b_{21} \\
0
\end{array}\right] \delta^{\prime}
$$

where the parameters are determined by the hydrodynamic coefficients, shown as

$$
\begin{aligned}
& a_{11}=\frac{\left(I_{Z}^{\prime}-N_{\dot{r}}^{\prime}\right) Y_{v}^{\prime}-\left(m^{\prime} x_{G}^{\prime}-Y_{\dot{r}}^{\prime}\right) N_{v}^{\prime}}{\left(m^{\prime}-Y_{\dot{v}}^{\prime}\left(I_{Z}^{\prime}-N_{\dot{r}}^{\prime}\right)\right)-\left(m^{\prime} x_{G}^{\prime}-Y_{\dot{r}}^{\prime}\right)\left(m^{\prime} x_{G}^{\prime}-N_{\dot{v}}^{\prime}\right)} \\
& a_{12}=\frac{\left(I_{Z}^{\prime}-N_{\dot{r}}^{\prime}\right)\left(Y_{r}^{\prime}-m^{\prime}\right)-\left(m^{\prime} x_{G}^{\prime}-Y_{\dot{r}}^{\prime}\right)\left(N_{r}^{\prime}-m^{\prime} x_{G}^{\prime}\right)}{\left(m^{\prime}-Y_{\dot{v}}^{\prime}\left(I_{Z}^{\prime}-N_{\dot{r}}^{\prime}\right)\right)-\left(m^{\prime} x_{G}^{\prime}-Y_{\dot{r}}^{\prime}\right)\left(m^{\prime} x_{G}^{\prime}-N_{\dot{v}}^{\prime}\right)} \\
& a_{21}=\frac{\left(Y_{r}^{\prime}-m^{\prime}\right) N_{v}^{\prime}-\left(m^{\prime} x_{G}^{\prime}-N_{\dot{v}}^{\prime}\right) Y_{v}^{\prime}}{\left(m^{\prime}-Y_{\dot{v}}^{\prime}\left(I_{Z}^{\prime}-N_{\dot{r}}^{\prime}\right)\right)-\left(m^{\prime} x_{G}^{\prime}-Y_{\dot{r}}^{\prime}\right)\left(m^{\prime} x_{G}^{\prime}-N_{\dot{v}}^{\prime}\right)} \\
& a_{22}=\frac{\left(m^{\prime}-Y_{\dot{v}}^{\prime}\right)\left(N_{r}^{\prime}-m^{\prime} x_{G}^{\prime}\right)-\left(m^{\prime} x_{G}^{\prime}-N_{\dot{v}}^{\prime}\right)\left(Y_{r}^{\prime}-m^{\prime}\right)}{\left.\left(m^{\prime}-Y_{\dot{v}}^{\prime}\right)\left(I_{Z}^{\prime}-N_{\dot{r}}^{\prime}\right)\right)-\left(m^{\prime} x_{G}^{\prime}-Y_{\dot{r}}^{\prime}\right)\left(m^{\prime} x_{G}^{\prime}-N_{\dot{v}}^{\prime}\right)} \\
& b_{11}=\frac{\left(I_{Z}^{\prime}-N_{\dot{r}}^{\prime}\right) Y_{\delta}^{\prime}-\left(m^{\prime} x_{G}^{\prime}-Y_{\dot{r}}^{\prime}\right) N_{\delta}^{\prime}}{\left.\left(m^{\prime}-Y_{\dot{v}}^{\prime}\right)\left(I_{Z}^{\prime}-N_{\dot{r}}^{\prime}\right)\right)-\left(m^{\prime} x_{G}^{\prime}-Y_{\dot{r}}^{\prime}\right)\left(m^{\prime} x_{G}^{\prime}-N_{\dot{v}}^{\prime}\right)} \\
& b_{21}=\frac{\left(m^{\prime}-Y_{\dot{v}}^{\prime}\right) N_{\delta}^{\prime}-\left(m^{\prime} x_{G}^{\prime}-N_{\dot{v}}^{\prime}\right) Y_{\delta}}{\left.\left(m^{\prime}-Y_{\dot{v}}^{\prime}\right)\left(I_{Z}^{\prime}-N_{\dot{r}}^{\prime}\right)\right)-\left(m^{\prime} x_{G}^{\prime}-Y_{\dot{r}}^{\prime}\right)\left(m^{\prime} x_{G}^{\prime}-N_{\dot{v}}^{\prime}\right)}
\end{aligned}
$$

Rewriting the state variables of (A.8) with a dimensional format yields

$$
\left[\begin{array}{c}
\dot{v} \\
\dot{r} \\
\dot{\psi}
\end{array}\right]=\left[\begin{array}{c}
a_{11} v \frac{U}{L}+a_{12} r U+b_{11} \delta \frac{U^{2}}{L} \\
a_{21} v \frac{U}{L^{2}}+a_{22} r \frac{U}{L}+b_{21} \delta \frac{U^{2}}{L^{2}} \\
r
\end{array}\right]
$$

Construction of Samples. The continuous (A.9) is discretized using the Euler forward method. Its difference form can be expressed as

$$
\left[\begin{array}{l}
v(k+1) \\
r(k+1)
\end{array}\right]=\left[\begin{array}{l}
v(k)+\frac{a_{11} \Delta t}{L} v(k) U(k)+a_{12} \Delta t r(k) U(k)+\frac{b_{11} \Delta t}{L} \delta(k) U^{2}(k) \\
r(k)+\frac{a_{21} \Delta t}{L^{2}} v(k) U(k)+\frac{a_{22} \Delta t}{L} r(k) U(k)+\frac{b_{21} \Delta t}{L^{2}} \delta(k) U^{2}(k)
\end{array}\right]
$$


where $k+1$ and $k$ denote two successive sampling times, $\Delta t$ is the sampling interval. Then the input-output pairs used by LS-SVR and RLS are expressed as follows.

The inputs are $\mathbf{Y}=\left[v(k), v(k) U(k), r(k) U(k), \delta(k) U^{2}(k)\right]_{4 \times 1}^{T}$, and

$\mathbf{Z}=\left[r(k), v(k) U(k), r(k) U(k), \delta(k) U^{2}(k)\right]_{4 \times 1}^{T}$. Let $\mathbf{B}_{\text {par }}=\left[\begin{array}{llll}1 & b_{1} & b_{2} & b_{3}\end{array}\right]_{1 \times 4}$,

$\mathbf{C}_{\text {par }}=\left[\begin{array}{llll}1 & c_{1} & c_{2} & c_{3}\end{array}\right]_{1 \times 4}$, then the outputs are $v(k+1)=\mathbf{B}_{\text {par }} \mathbf{Y}$, and $r(k+1)=\mathbf{C}_{p a r} \mathbf{Z}$. Once the parameters in $\mathbf{B}_{p a r}$ and $\mathbf{C}_{p a r}$ are estimated, the parameters of the state space model (A.8) can be achieved immediately through the following conversions.

$$
a_{11}=\frac{b_{1} L}{\Delta t}, a_{12}=\frac{b_{2} L}{\Delta t}, b_{11}=\frac{b_{3} L}{\Delta t}, a_{21}=\frac{c_{1} L^{2}}{\Delta t}, a_{22}=\frac{b_{2} L}{\Delta t}, b_{21}=\frac{c_{1} L^{2}}{\Delta t}
$$

\section{A.4 A 4 DOF Nonlinear Model of a Large Container Ship}

The nonlinear roll-coupled 4 DOF model for the high-speed container ship is written as (Son and Nomoto 1982)

$$
\begin{gathered}
\left(m-X_{\dot{u}}\right) \dot{u}-\left(m-Y_{\dot{v}}\right) v r=X \\
\left(m-Y_{\dot{v}}\right) \dot{v}+\left(m-X_{\dot{u}}\right) u r-Y_{\dot{r}} \dot{r}=Y \\
\left(I_{x}-K_{\dot{p}}\right) \dot{p}=K-W G \bar{M}_{T} \phi \\
\left(I_{z}-K_{\dot{r}}\right) \dot{r}+N_{\dot{v}} \dot{v}=N
\end{gathered}
$$

where

$$
\begin{aligned}
X= & X(u)+(1-t) T+X_{v r} v r+X_{v v} v^{2}+X_{r r} r^{2}+X_{\phi \phi} \phi^{2}+X_{\delta} \sin \delta+X_{e x t} \\
Y= & Y_{v} v+Y_{r} r+Y_{\phi} \phi+Y_{p} p+Y_{v v v} v^{3}+Y_{r r r} r^{3}+Y_{v v r} v^{2} r+Y_{v r r} v r^{2}+Y_{v v \phi} v^{2} \phi \\
& +Y_{v \phi \phi} \phi^{2} v+Y_{r r \phi} r^{2} \phi+Y_{\delta} \cos \delta+Y_{e x t} \\
K= & K_{v} v+K_{r} r+K_{\phi} \phi+K_{p} p+K_{v v v} v^{3}+K_{r r r} r^{3}+K_{v v r} v^{2} r+K_{v r r} v r^{2}+K_{v \phi \phi} \phi^{2} v \\
& +K_{v v \phi} v^{2} \phi+K_{r r \phi} r^{2} \phi+Y_{r \phi \phi} r \phi^{2}+K_{\delta} \cos \delta+K_{e x t} \\
N= & N_{v} v+N_{r} r+N_{\phi} \phi+N_{p} p+N_{v v v} v^{3}+N_{r r r} r^{3}+N_{v v r} v^{2} r+N_{v r r} v r^{2} \\
& +N_{v \phi \phi} \phi^{2} v+N_{v v \phi} v^{2} \phi+N_{r r \phi} r^{2} \phi+N_{r \phi \phi} r \phi^{2}+N_{\delta} \cos \delta+N_{e x t}
\end{aligned}
$$

The variants are used in accordance with SNAME. $\dot{u}$ and $\dot{v}$ are linear accelerations in the $x$ and $y$ direction, respectively, $\dot{p}$ and $\dot{r}$ are angular accelerations in the $x$ and $z$ direction, respectively, $I_{x}$ and $I_{z}$ are the inertial moments about the $x$ and $z$ axis, respectively, $m$ is the mass of the ship, $W$ is the weight of water displaced by the ship's hull, $G \bar{M}_{T}$ is the transverse metacentric height, $X(u)$ is the velocity dependent damping function, $t$ is the thrust deduction factor, $T$ is the propeller thrust and $\delta$ is the rudder angle , $X_{e x t}, Y_{e x t}, K_{e x t}$ and $N_{e x t}$ are forces or moments due to external 
disturbances. The left nomenclatures are hydrodynamic derivatives, see (Rajesh and Bhattacharyya 2008) for related details. The principal particulars of the container ship are given in Table A.4.1.

Table A.4.1: Principal particulars of the container ship.

\begin{tabular}{ll}
\hline Particulars & Values \\
\hline Length between perpendiculars & $175 \mathrm{~m}$ \\
Length over all & $178 \mathrm{~m}$ \\
Breadth & $25.4 \mathrm{~m}$ \\
Draft at the fore end & $8 \mathrm{~m}$ \\
Draft at the aft end & $9 \mathrm{~m}$ \\
Mean draft & $8.5 \mathrm{~m}$ \\
Displacement volume & $21,222 \mathrm{~m}^{3}$ \\
Height from keel to transverse metacenter & $10.39 \mathrm{~m}$ \\
Height from keel to center of buoyancy & $4.6145 \mathrm{~m}$ \\
Block coefficient & 0.559 \\
Rudder area & $33.0376 \mathrm{~m}^{2}$ \\
Aspect ratio & 1.8219 \\
Propeller diameter & $6.533 \mathrm{~m}$ \\
\hline
\end{tabular}




\section{References}

Martin A Abkowitz. Lectures on ship hydrodynamics-steering and manoeuvrability. Technical report, Stevens Institute of Technology, 1964.

Motoki Araki, Hamid Sadat-Hosseini, Yugo Sanada, Kenji Tanimoto, Naoya Umeda, and Frederick Stern. Estimating maneuvering coefficients using system identification methods with experimental, system-based, and cfd free-running trial data. Ocean Engineering, 51 : 63-84, 2012.

Karl J Åström. Design of fixed gain and adaptive ship steering autopilots based on the nomoto model. In Proceedings, Symposium on Ship Steering Automatic Control, Genova, 1980.

Karl J Åström and Claes G Källström. Identification of ship steering dynamics. Automatica, 12 (1):9-22, 1976.

Mogens Bech and Wagner L Smitt. Analogue simulation of ship maneuvers based on full scale trials or free sailing model tests. Technical report, Stevens Institute of Technology, 1969.

W. Beukelman and J.M.J. Journée. Hydrodynamic transverse loads on ships in deep and shallow water. In Proceedings of the 22nd International Conference HADMAR, pages 1-4, 2001.

Sriman K Bhattacharyya and Mahmoud R Haddara. Parametric identification for nonlinear ship maneuvering. Journal of ship research, 50(3):197-207, 2006.

Mogens Blanke. Ship propulsion losses related to automatic steering and prime mover control. $\mathrm{PhD}$ thesis, Technical University of Denmark, 011981.

Andrew W Browning. A mathematical model to simulate small boat behaviour. Simulation, 56 (5):329-336, 1991.

Christopher JC Burges. A tutorial on support vector machines for pattern recognition. Data mining and knowledge discovery, 2(2):12 1-167, 1998.

Massimo Caccia, Gabriele Bruzzone, and Riccardo Bono. A practical approach to modeling and identification of small autonomous surface craft. IEEE Journal of Oceanic Engineering, 33(2): 133-145, 2008.

A.C. Christensen and Mogens Blanke. A Linearized State-space Model of Steering and Roll of a High-speed Container Ship. Technical University of Denmark (DTU), 1986.

Kenneth SM Davidson and Leonard I Schiff. Turning and course-keeping qualities of ships. Trans. of Soc.of Naval Arch. and Marine Engineers, 1964. 
Christian Denker. Assessing the spatio-temporal fitness of information supply and demand on an adaptive ship bridge. In International Conference on Knowledge Engineering and Knowledge Management, pages 185-192. Springer, 2014.

Laura Dioşan, Alexandrina Rogozan, and Jean-Pierre Pecuchet. Improving classification performance of support vector machine by genetically optimising kernel shape and hyper-parameters. Applied Intelligence, 36(2):280-294, 2012.

Izzet Kagan Erunsal. System identification and control of a sea surface vehicle. Dept. Elec. and Electronics Eng., Middle East Technical Uni., 2015.

Odd M Faltinsen. Hydrodynamics of high-speed marine vehicles. Cambridge university press, 2005.

K.K. Fedyaevsky and G.V. Sobolev. Control and stability in ship design. State Union Shipbuilding Industry Publishing House, 1964.

Gabriele Ferri, Alessandro Manzi, Francesco Fornai, Francesco Ciuchi, and Cecilia Laschi. A systematic method for dynamic modeling and identification of a small-sized autonomous surface vehicle using simulated annealing techniques. In OCEANS-Bergen, 2013

MTS/IEEE, pages 1-9. IEEE, 2013.

Thor I Fossen. Guidance and control of ocean vehicles. John Wiley \& Sons Inc, 1994.

Thor I Fossen. Handbook of marine craft hydrodynamics and motion control. John Wiley \& Sons, 2011.

Thor I Fossen. Lecture notes ttk4190 guidance and control of vehicles, 2013.

Holger Frohlich and Andreas Zell. Efficient parameter selection for support vector machines in classification and regression via model-based global optimization. In Neural Networks, 2005. IJCNN'o 5. Proceedings. 2005 IEEE International Joint Conference on, volume 3, pages 1431-1436. IEEE, 2005.

$\mathrm{Li} \mathrm{Fu}$ and Pengfei Li. The research survey of system identification method. In Intelligent Human-Machine Systems and Cybernetics (IHMSC), 2013 5th International Conference on, volume 2, pages 397-401. IEEE, 2013.

KR Godfrey and JJ DiStefano III. Identifiability of model parameter. IFAC Proceedings Volumes, $18(5): 89-114,1985$.

Axel Hahn. Test bed for safety assessment of new e-navigation systems. International journal of e-Navigation and Maritime Economy, 1:14-28, 2014.

Axel Hahn, Volker Gollücke, Carsten Buschmann, and Sören Schweigert. Virtual test bed for maritime safety assessment. Zeszyty Naukowe Akademii Morskiej w Szczecinie, 2015.

Elias Revestido Herrero and Francisco J Velasco Gonzalez. Two-step identification of non-linear manoeuvring models of marine vessels. Ocean Engineering, 53:72-82, 2012.

Masayoshi Hirano. A practical calculation method of ship maneuvering motion at initial design stage. Naval architecture and ocean engineering, 19:68-80, 1981.

Thomas Hofmann, Bernhard Schölkopf, and Alexander J Smola. Kernel methods in machine learning. The annals of statistics, pages 1171-1220, 2008. 
J.P. Hooft. The cross-flow drag on a manoeuvring ship. Ocean engineering, 21 (3):329-342, 1994. Xian-Rui Hou and Zao-Jian Zou. Parameter identification of nonlinear roll motion equation for floating structures in irregular waves. Applied Ocean Research, 55:66-75, 2016.

Wei-Yuan Hwang. Application of system identification to ship maneuvering. $\mathrm{PhD}$ thesis, Massachusetts Institute of Technology, 1980.

Rob J Hyndman and Anne B Koehler. Another look at measures of forecast accuracy. International journal of forecasting, 22 (4):679-688, 2006.

IMO. E-navigation. http:

//www.imo.org/en/OurWork/Safety/Navigation/Pages/eNavigation.aspx, 2014. Accessed: 2019-02-18.

Shosuke Inoue, Masayoshi Hirano, and Katsuro Kijima. Hydrodynamic derivatives on ship manoeuvring. International Shipbuilding Progress, 28(321):112-1 25, 1981.

Cao Jian, Zhuang Jiayuan, Xu Feng, Yin Jianchuan, Zou Zaojian, Yu Hao, Xiao Tao, and Yang Luchun. Parametric estimation of ship maneuvering motion with integral sample structure for identification. Applied Ocean Research, 52:212-221, 2015.

Dervis Karaboga. An idea based on honey bee swarm for numerical optimization. Technical report, Technical report-tro6, Erciyes university, engineering faculty, computer engineering department, 2005 .

Katsuro Kijima. On the practical prediction method for ship manoeuvring characteristics. Transactions of the West-Japan Society of Naval Architects, pages 21-31, 2002.

Katsuro Kijima and Yasuaki Nakiri. Prediction method of ship manoeuvrability in deep and shallow waters. In MARSIM \& ICSM 9o, Intl. Conference, Marine Simulation and Ship Manoeuvrability, pages 311-318, 1990.

Katsuro Kijima, Toshiyuki Katsuno, Yasuaki Nakiri, and Yoshitaka Furukawa. On the manoeuvring performance of a ship with theparameter of loading condition. Journal of the society of naval architects of Japan, 1990(168):141-148, 1990.

E Kobayashi, H Kagemoto, and Y Furukawa. Research on ship manoeuvrability and its application to ship design. chapter 2: mathematical models of manoeuvring motions. In The 12 th Marine Dynamic Symposium, pages 23-90, 1995.

Edward V Lewis. Principles of naval architecture second revision, volume iii. Jersey City: The Society of Naval Architects and Marine Engineers, 601:14-40, 1988.

Karl-Petter Lindegaard and Thor I Fossen. Fuel-efficient rudder and propeller control allocation for marine craft: Experiments with a model ship. IEEE Transactions on Control Systems Technology, $11(6): 850-862,2003$.

Zhixiang Liu, Youmin Zhang, Xiang Yu, and Chi Yuan. Unmanned surface vehicles: An overview of developments and challenges. Annual Reviews in Control, 41:71-93, 2016.

Lennart Ljung. System identification. In Signal analysis and prediction, pages 163-173. Springer, 1998. 
Weilin Luo. Parameter identifiability of ship manoeuvring modeling using system identification. Mathematical Problems in Engineering, 2016, 2016.

Weilin Luo and Hongchao Cong. Control for ship course-keeping using optimized support vector machines. Algorithms, 9(3):52, 2016.

Weilin Luo, C Guedes Soares, and Zaojian Zou. Parameter identification of ship maneuvering model based on support vector machines and particle swarm optimization. Journal of Offshore Mechanics and Arctic Engineering, 138(3):031101, 2016.

Wwilin. Luo and Zaojian Zou. Parametric identification of ship maneuvering models by using support vector machines. Journal of Ship Research, 53(1):19-30, 2009.

David Moreno-Salinas, Dictino Chaos, Jesús Manuel de la Cruz, and Joaquín Aranda. Identification of a surface marine vessel using ls-svm. Journal of Applied Mathematics, 2013, 2013 .

John Nicholas Newman. Marine hydrodynamics. MIT press, 2018.

Hung Duc Nguyen. Recursive identification of ship manoeuvring dynamics and hydrodynamics. ANZIAM Journal, 49:717-732, 2008.

Kensaku Nomoto, Kenshi Taguchi, Keinosuke Honda, and Susumu Hirano. On the steering qualities of ships. International Shipbuilding Progress, 4(35):354-370, 1957.

N. H. Norrbin. On the design and analysis of the zig-zag test on base of quasi-linear frequency response. Technical Report SSPA B 104-3, Stevens Institute of Technology, 1963.

N. H. Norrbin. Theory and observations on the use of a mathematical model for ship manoeuvring in deep and confined waters. Technical report, Swedish State Shipbuilding Experimental Tank Goteborg, 1971.

Tristan Pérez and Mogens Blanke. Mathematical ship modeling for control applications. Technical report, Technical University of Denmark, 2002.

Tristan Pérez and Thor I Fossen. Time-domain models of marine surface vessels for simulation and control design based on seakeeping computations. In Proc. 7 th IFAC Conference on Manoeuvring and Control of Marine Craft MCMC. Lisbon, Portugal, 2006.

John C Platt. Fast training of support vector machines using sequential minimal optimization, advances in kernel methods. Support Vector Learning, pages 185-208, 1999.

Stephen J Pullard. Recursive parameter identification for estimating and displaying maneuvering vessel path. Technical report, NAVAL POSTGRADUATE SCHOOL MONTEREY CA, 2003.

Benjamin Racine and Eric Paterson. Cfd-based method for simulation of marine-vehicle maneuvering. In 35th AIAA Fluid Dynamics Conference and Exhibit, page 4904, 2005.

G. Rajesh and S.K. Bhattacharyya. System identification for nonlinear maneuvering of large tankers using artificial neural network. Applied Ocean Research, 30(4):256-263, 2008. 
Key Pyo Rhee, Hyeon Kyu Yoon, Young Jae Sung, Sung Hoon Kim, and Ju Nyun Kang. An experimental study on hydrodynamic coefficients of submerged body using planar motion mechanism and coning motion device. In International Workshop on Ship Manoeuvrability at the Hamburg Ship Model Basin, pages 1-20, 2000.

Bernhard Schiilkop, Chris Burgest, and Vladimir Vapnik. Extracting support data for a given task. In Proceedings, First International Conference on Knowledge Discovery \& Data Mining. AAAI Press, Menlo Park, CA, pages 252-257, 1995.

Bernhard Scholkopf and Alexander J Smola. Learning with kernels: support vector machines, regularization, optimization, and beyond. MIT press, 2002.

R Rajita Shenoi, P Krishnankutty, and R Panneer Selvam. Sensitivity study of hydrodynamic derivative variations on the maneuverability prediction of a container ship. In ASME 2015 34th International Conference on Ocean, Offshore and Arctic Engineering, pages Voo7To6Aoo8-Voo7To6Aoo8. American Society of Mechanical Engineers, 2015.

Alexandre N Simos, Celso P Pesce, Michael M Bernitsas, and Stuart B Cohen. Hydrodynamic model induced differences in spm post pitchfork bifurcation paths. Journal of Offshore Mechanics and Arctic Engineering, 124(4):174-178, 2002.

Roger Skjetne, Øyvind N Smogeli, and Thor I Fossen. A nonlinear ship manoeuvering model: Identification and adaptive control with experiments for a model ship. Modeling, Identification and Control, 25 (1):3-27, 2004.

Alex J Smola and Bernhard Schölkopf. A tutorial on support vector regression. Statistics and computing, 14(3):199-222, 2004.

SNAME. Nomenclature for treating the motion of a submerged body through a fluid. New York: Technical and Research Bulletin, pages 1-5, 1950.

Kyoung-Ho Son and Kensaku Nomoto. 5. on the coupled motion of steering and rolling of a high-speed container ship. Naval Architecture and Ocean Engineering, 20:73-83, 1982.

Christian R Sonnenburg and Craig A Woolsey. Modeling, identification, and control of an unmanned surface vehicle. Journal of Field Robotics, 30(3):371-398, 2013.

Mohd Herwan Sulaiman, Mohd Wazir Mustafa, Hussain Shareef, and Saiful Nizam Abd Khalid. An application of artificial bee colony algorithm with least squares support vector machine for real and reactive power tracing in deregulated power system. International Journal of Electrical Power \& Energy Systems, 37(1):67-77, 2012.

Serge Sutulo and C Guedes Soares. An algorithm for offline identification of ship manoeuvring mathematical models from free-running tests. Ocean Engineering, 79:10-25, 2014.

Johan AK Suykens and Joos Vandewalle. Least squares support vector machine classifiers. Neural processing letters, 9(3):293-300, 1999.

Johan A.K. Suykens, Tony Van Gestel, Jos De Brabanter, Bart De Moor, and Joos Vandwalle. Least squares support vector machines. World Scientific, 2002. 
Michael E Taylor. System identification and control of an arleigh burke class destroyer using an extended kalman filter. Technical report, MASSACHUSETTS INST OF TECH CAMBRIDGE DEPT OF MECHANICAL ENGINEERING, 2000.

Serge Leon Toxopeus. Practical application of viscous-flow calculations for the simulation of manoeuvring ships. PhD thesis, Technische Universiteit Delft, 2011.

Vladimir Vapnik. The nature of statistical learning theory. Springer science \& business media, 2013.

Vladimir Vapnik, Steven E Golowich, and Alex J Smola. Support vector method for function approximation, regression estimation and signal processing. In Proceedings of the 9 th International Conference on Neural Information Processing Systems, NIPS'96, pages 28 1-287, Cambridge, MA, USA, 1996. MIT Press. URL http : //dl . acm.org/citation. cfm?id=2998981 . 2999021.

Xuegang Wang, Zaojian Zou, Zhaolong Yang, and Feng Xu. Sensitivity analysis of the hydrodynamic coefficients in 4 degrees of freedom ship manoeuvring mathematical model. Journal of Shanghai Jiaotong University (Science), 20(5):584-590, 2015.

Tao Xiong, Yukun Bao, and Zhongyi Hu. Multiple-output support vector regression with a firefly algorithm for interval-valued stock price index forecasting. Knowledge-Based Systems, 55: $87-100,2014$.

Dong Jin Yeo and Key Pyo Rhee. Sensitivity analysis of submersibles' manoeuvrability and its application to the design of actuator inputs. Ocean engineering, 33(17-18):2270-2286, 2006.

Hyeon Kyu Yoon and Key Pyo Rhee. Identification of hydrodynamic coefficients in ship maneuvering equations of motion by estimation-before-modeling technique. Ocean Engineering, 30(18):2379-2404, 2003.

Xinguang Zhang and Zaojian Zou. Estimation of the hydrodynamic coefficients from captive model test results by using support vector machines. Ocean Engineering, 73:2 5-31, 2013. 


\section{Statement}

I, Man Zhu, hereby assure that I have written the present work about Optimized Support Vector Regression Algorithm-based Modeling of Ship Dynamics independently and have not used any sources other than those specified and that I have followed the general principles of scientific work and publications, as defined in the guidelines of good academic practice of the Carl von Ossietzky University of Oldenburg.

Oldenburg, February 20, 2019

Man Zhu 